\title{
X-ray binaries in the Milky Way and other galaxies
}

Hans-Jakob Grimm  



\title{
X-ray binaries in the Milky Way and other galaxies
}

Hans-Jakob Grimm

\author{
Dissertation \\ an der Fakultät für Physik \\ der Ludwig-Maximilians-Universität \\ München
}

vorgelegt von

Hans-Jakob Grimm

aus Rostock

München, den 28.03.2003 
Erstgutachter: Prof. Dr. Rashid Sunyaev Zweitgutachter: Prof. Dr. Ralf Bender Tag der mündlichen Prüfung: 04.08.2003 


\section{Contents}

Zusammenfassung $\quad$ xi

1. Introduction $\quad 1$

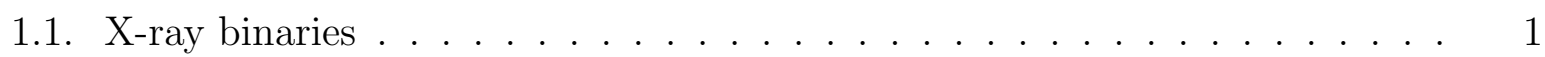

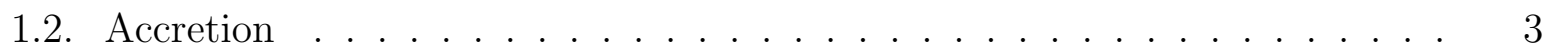

1.3. Motivation and Outline . . . . . . . . . . . . . 5

1.3.1. Galactic X-ray binary population . . . . . . . . . . 5

1.3.2. HMXB-SFR connection . . . . . . . . . . . . . . 7

$\begin{array}{ll}\text { I. The Milky Way } & 11\end{array}$

2. Milky Way $\log (\mathrm{N})-\log (\mathrm{S}) \quad 13$

2.1. RXTE All-Sky Monitor Data . . . . . . . . . . . . . . . . . 13

2.1.1. Systematic errors . . . . . . . . . . . . . . . . . 13

2.1.2. Completeness . . . . . . . . . . . . . . . . . 15

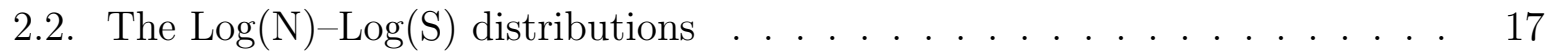

3. Milky Way Luminosity functions

3.1. Distribution of X-ray binaries in the Galaxy . . . . . . . . . . . . . . . . . 21

3.1.1. Angular distribution of X-ray binaries . . . . . . . . . . . . . . . . 21

3.1.2. Source distances and 3-D distribution of X-ray binaries . . . . . . . 21

3.1.3. The Galaxy model . . . . . . . . . . . . . . . . . . . 23

3.1.4. High mass X-ray binaries . . . . . . . . . . . . . . . . . 25

3.1.5. Low mass X-ray binaries . . . . . . . . . . . . . . . . . . . 27

3.1.6. Completeness of the sample of the distance measurements. . . . . . 28

3.2. Luminosity function . . . . . . . . . . . . . . . . . . . . . . 29

3.2.1. Effect of the Galaxy model on the luminosity function . . . . . . . 32

3.2.2. Total X-ray luminosity of Galactic X-ray binaries . . . . . . . . . . 33

3.2.3. Luminosity function and $\dot{M}$ distribution of X-ray binaries . . . . . . 35 
4. Extension of luminosity functions $\quad 41$

4.1. Low luminosity sources . . . . . . . . . . . . . . . . . . . . . . 41

4.1.1. Extension of $\log (\mathrm{N})-\log (\mathrm{S})$ towards lower fluxes . . . . . . . . . . 41

4.1.2. Low luminosity end of X-ray binary luminosity function . . . . . . . 42

4.1.3. Young objects in star forming regions . . . . . . . . . . . . . . . . 44

4.2. High luminosity sources . . . . . . . . . . . . . . . . . . . . 44

II. Star-forming galaxies $\quad 47$

5. Data on star forming galaxies $\quad 49$

5.1. Sample of galaxies . . . . . . . . . . . . . . . . . . . . . . . 49

5.1.1. Distances . . . . . . . . . . . . . . . . 52

5.1.2. X-ray luminosity functions . . . . . . . . . . . . . . . . . . . . 52

5.1.3. Star formation rate estimates . . . . . . . . . . . . . 54

5.1.4. Contribution of a central AGN . . . . . . . . . . . . 56

5.1.5. Contribution of LMXBs . . . . . . . . . . . . . . . . 57

6. High Mass X-ray Binaries as a star formation indicator 59

6.1. Universal HMXB Luminosity Function ? . . . . . . . . . . . . . . . . . . 59

6.2. High Luminosity cut-off . . . . . . . . . . . . . . . . . . 65

6.3. Total X-ray luminosity as SFR indicator . . . . . . . . . . . . . . 66

6.4. Theoretical $L_{X}-\mathrm{SFR}$ relation . . . . . . . . . . . . . . . . . . . . . 66

6.5. $L_{X}$-SFR relation: comparison with the data . . . . . . . . . . . . . 69

6.6. Hubble Deep Field North . . . . . . . . . . . . . . . . . . . . . 70

6.7. Discussion . . . . . . . . . . . . . . . . . . . 71

6.7.1. Neutron stars, stellar mass black holes and intermediate mass black holes ........................ . . 71

6.7.2. Further astrophysically important information . . . . . . . . . . 76

6.8. Collective luminosity of a population of discrete sources . . . . . . . . . . 78

6.8.1. Analytical treatment . . . . . . . . . . . . . . . . . 79

$\begin{array}{ll}\text { 7. Summary } & 87\end{array}$

$\begin{array}{ll}\text { 8. Appendix } & 91\end{array}$

$\begin{array}{ll}\text { Bibliography } & 101\end{array}$ 


\section{List of Figures}

2.1. Angular distribution of LMXBs and HMXBs in the Galaxy. . . . . . . . . 14

2.2. Systematic error estimate using observed versus expected RMS for 10 long term stable sources. . . . . . . . . . . . . . . . . . 15

2.3. $\log (\mathrm{N})-\log (\mathrm{S})$ distribution of extragalactic sources. . . . . . . . . . . . 16

2.4. Number-flux relation for all galactic sources derived from the entire ASM sample. . . . . . . . . . . . . . . . . . . . 17

2.5. $\log (\mathrm{N})-\log (\mathrm{S})$ for Galactic X-ray binaries. . . . . . . . . . . . . . . 18

3.1. Distribution of Galactic X-ray binaries versus Galactic longitude and latitude. 22

3.2. Face-on view of the Galaxy. . . . . . . . . . . . . . . . . . . . . 23

3.3. Radial distributions of HMXBs and LMXBs. . . . . . . . . . . . . . . . 24

3.4. Vertical distributions of HMXBs and LMXBs. . . . . . . . . . . . . . . . . 25

3.5. Distribution of LMXB sources over distance from the Sun. . . . . . . . . . 29

3.6. Fraction of mass of the Galaxy visible to ASM. . . . . . . . . . . . . . 30

3.7. Apparent and volume corrected cumulative luminosity function. . . . . . . 31

3.8. Apparent and volume corrected differential luminosity function. . . . . . . 31

3.9. Dependence of the luminosity function on the adopted model of the spatial distribution of XRBs. . . . . . . . . . . . . . . . . . . 33

4.1. Comparison of the differential $\log (\mathrm{N})-\log (\mathrm{S})$ relation for Galactic XRBs

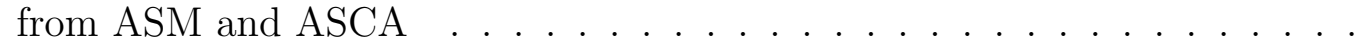

4.2. Comparison of the number-flux relation observed in the ASCA GRS and the predicted $\log (\mathrm{N})-\log (\mathrm{S})$ from ASM luminosity functions. . . . . . . . . .

4.3. Spatial distribution of Galactic XRBs with episodes of Eddington or superEddington luminosity. . . . . . . . . . . . . . . .

5.1. The luminosity functions of compact X-ray sources in nearby galaxies from the primary sample in Table $5.1 . \ldots \ldots \ldots$. . . . . . . . .

5.2. Contributions of LMXBs and HMXBs to the luminosity function of NGC 4736 .

6.1. Number-SFR relation for galaxies from Table 5.1. . . . . . . . . . . . 60

6.2. Comparison of the combined luminosity function of M 82, NGC 4579, NGC 4736 and Circinus with luminosity function of Antennae. 
6.3. Combined luminosity function of star-burst galaxies. . . . . . . . . . . 62

6.4. Comparison of "universal" luminosity function with individual galaxies. . . 64

6.5. The $L_{X}$-SFR relation. . . . . . . . . . . . . . . . . . . . . . . . . . 69

6.6. Simulated differential luminosity function. . . . . . . . . . . . . 73

6.7. Probability distributions of average luminosity of discrete sources for various luminosity function slopes . . . . . . . . . . . . . . . . 81

6.8. Dependence of most probable value on number of the sources. . . . . . . . 82

6.9. Ratio of most probable value of total luminosity to its expectation value versus number of sources. . . . . . . . . . . . . . . . 83

6.10. Probability distribution of the maximum luminosity. . . . . . . . . . . . . 84 


\section{List of Tables}

2.1. List of sources used to estimate systematic errors. . . . . . . . . . . . . 14

2.2. Best fit values for $\log (\mathrm{N})-\log (\mathrm{S})$ of Galactic sources. . . . . . . . . . . . 19

3.1. The parameters of the standard Galaxy model. . . . . . . . . . . . . . 26

3.2. Most luminous LMXB sources. . . . . . . . . . . . . . . . . . . . . 34

3.3. Most luminous HMXB sources. . . . . . . . . . . . . . . . . . . . . . . . . . . . . . . . . . . . . . 35

3.4. X-ray binaries with super-Eddington flux periods. . . . . . . . . . . 38

5.1. The primary sample of local galaxies used to study the luminosity function of HMXB sources. . . . . . . . . . . . . . . . . . . 50 50

5.2. The secondary sample of local galaxies used to complement the primary sample in the analysis of the $L_{X}$-SFR relation. . . . . . . . . . . . . . 51

5.3. Star formation rates for galaxies from local sample. . . . . . . . . . . . . 55

6.1. Sample galaxies from the Hubble Deep Field North and Lynx Field. . . . . 71

8.1. List of all galactic sources observed with ASM . . . . . . . . . . . 91 


\section{Zusammenfassung}

Diese Arbeit beschäftigt sich mit der Population von Röntgendoppelsternen in der Milchstraße und dem Zusammenhang der Sternentstehungsrate einer Galaxis mit dem Auftreten Sternsysteme mit einem massiven optischen Begleiter.

Aufgrund ihrer speziellen Natur sind Röntgendoppelsterne für viele verschiedene Bereiche der Physik und Astrophysik von Interesse. Dies reicht von der Beschreibung der Verhältnisse innerhalb einer Akkretionsscheibe mittels der Methoden der Plasmaphysik bis zur Untersuchung stellarer Populationen in einer Galaxis.

Röntgendoppelsterne bestehen aus einem kompakten Objekt, einem Neutronenstern oder Schwarzen Loch, das als Primärobjekt bezeichnet wird, und einem stellaren Begleiter, dem Sekundärobjekt, der durch alle Sterntypen repräsentiert sein kann, von einem Braunen Zwerg über Hauptreihensterne bis zu Weißen Zwergen. Aufgrund seiner starken Gravitation entzieht das Primärobjekt seinem Begleiter Masse, die es zumindest teilweise akkretiert. Während die Materie sich im Gravitationspotential auf das Primärobjekt zubewegt, wird aufgrund magnetischer Viskosität Energie freigesetzt, die letztlich als elektromagnetische Strahlung emittiert wird. Die Strahlung wird dabei immer höherenergetischer je näher die Abstrahlung am Primärobjekt erfolgt. Aufgrund der Tiefe des Potentialwalles werden in Röntgendoppelsternen Photonen mit Energien von einigen Kiloelektronvolt, d.h. Röntgenstrahlung, emittiert.

Der Massentransfer kann auf zwei verschiedene Weisen stattfinden. Wenn der Begleitstern groß genug ist und sein Rochevolumen ausfüllt, fließt Materie über den inneren Lagrangepunkt des Systems in das Rochevolumen des kompakten Objekts. Aufgrund des nicht vernachlässigbaren Drehimpulses der Materie bildet sich eine Akkretionsscheibe um das kompakte Objekt. Innerhalb der Scheibe kommt es durch magnetische Reibung zu einem Transport von Drehimpuls von innen nach außen, wodurch ein Großteil der Materie sich auf das kompakte Objekt zubewegt. Eine weitere Möglichkeit des Massentransfers bietet ein Sternwind. In diesem Fall verliert der optische Stern Masse über einen sogenannten stellaren Wind, d.h. Materie wird über magnetische Effekte vom Stern weg beschleunigt. Das kompakte Objekt kann einen Teil dieses Windes einfangen. Wie weit der Einfluß des kompakten Objekts dabei reicht, hängt einerseits von der Masse des kompakten Objekts ab, und andererseits von der Geschwindigkeit des Windes. Je massiver das kompakte Objekt, desto weiter reicht sein Einfluß, und je schneller der Wind ist, desto kleiner ist der Radius innerhalb dessen Windmaterie akkretiert wird.

Aufgrund der mannigfaltigen Parameter eines Röntgendoppelsterns gibt es zahlreiche Klassifizierungsschemata abhängig von der Eigenschaft, an der man interessiert ist wie z.B. die Natur des kompakten Objekts, die Röntgenaktivität oder die Natur des Sekundär- 
objekts. In der vorliegenden Arbeit ist besonders die Unterteilung an Hand des Sekundärobjekts von Bedeutung. Grob kann man Röntgendoppelsterne so in zwei bis drei Kategorien einteilen. Röntgendoppelsterne mit einem Begleiter schwerer als ungefähr 5 Sonnenmassen sogenannte "High-mass X-ray binaries" (HMXB) und Röntgendoppelsterne mit einem Begleiter unter einer Sonnenmasse "Low-mass X-ray binaries" (LMXB). Für Röntgendoppelsterne mit Begleitern zwischen diesen Massenbereichen gibt es die Bezeichnung "Intermediate-mass X-ray binaries"(IMXB). Allerdings ist diese Kategorie in der Milchstraße kaum vorhanden.

Dieses Klassifizierungsschema trägt auch der Lebenserwartung eines Röntgendoppelsterns Rechnung. Da die Zeit, die ein Stern als Hauptreihenstern verbringt ungefähr umgekehrt proportional zum Quadrat der Masse ist, unterscheiden sich die Lebenserwartung eines Sterns mit einer Sonnenmasse, ca. 10 Milliarden Jahre, und die eines Sterns mit 10 Sonnenmassen, 100 Millionen Jahre, um einen Faktor 100. Die kürzere Lebenszeit hat wichtige Implikationen für den Röntgendoppelstern. Röntgendoppelsterne können sich nicht weit von dem Ort ihrer Entstehung entfernen und die gesamte Population ist abhängig von der Sternentstehungsrate. Daher sind HMXBs sowohl eine örtliche als auch zeitliche Spur von Sternentstehung.

Die vorliegende Arbeit unterteilt sich in zwei Teile. Im ersten Teil beschäftige ich mich mit der Verteilung und den Eigenschaften von Röntgendoppelsternn in der Milchstraße. Der zweite Teil der Arbeit ist den Korrelationen von HMXBs und der Sternentstehungsrate in einer Galaxis gewidmet. Mit Hilfe der Daten des hochauflösenden Chandra-Röntgenobservatoriums ist es möglich, Röntgendoppelsterne in anderen Galaxien zu beobachten und damit die Zusammenhänge zwischen der Population von Röntgendoppelsternen und den Verhältnissen in diesen Galaxis zu untersuchen.

Eine hinreichend detaillierte Untersuchung der Verteilung von Röntgendoppelsternen in der Milchstraße ist erst in letzter Zeit möglich geworden, da bis vor wenigen Jahren nicht genügend Entfernungsbestimmungen und/oder optische Beobachtungen für eine größere Zahl von Röntgendoppelsternen verfügbar waren. Der Fortschritt bei Entfernungsbestimmungen erlaubt nun erstmals, die dreidimensionale Verteilung von Röntgendoppelsternen in unserer Milchstraße zu untersuchen. Dies erlaubt den Vergleich des Erscheinungsbildes unserer Milchstraße mit Beobachtungen des Chandra-Röntgenteleskops von anderen Galaxien. Außerdem können Rückschlüsse auf die Entstehung von Sternpopulationen gezogen werden. Beispielsweise ist die Verteilung von LMXBs in der Milchstraße stark im galaktischen Zentrum konzentriert, aber LMXBs sind in allen Teilen der Milchstraße vorhanden, HMXBs dagegen sind lediglich in der Milchstraßenscheibe zu finden. Diese Beobachtung ist im Einklang mit der Erwartung, daß der größte Teil der Sternentstehung in der Milchstraße in der galaktischen Scheibe stattfindet. Außerdem ist die Verteilung von HMXBs und LMXBs in der galaktischen Scheibe verschieden. HMXBs haben eine Skalenhöhe senkrecht zur galaktischen Scheibe von lediglich 150 pc, während LMXBs eine Skalenhöhe von 410 pc aufweisen. Darüberhinaus weist die räumliche Verteilung von Röntgendoppelsternen Spuren der Spiralstruktur der galaktischen Scheibe auf.

Die zahlreich vorhandenen Entfernungsbestimmungen erlauben zudem erstmals die Er- 
stellung einer Leuchtkraftverteilung von Röntgendoppelsternen in der Milchstraße. Dies ist von besonderer Bedeutung für den Vergleich mit anderen Galaxien, da in diesen Fällen aufgrund der großen Entfernungen die beobachtete Helligkeitsverteilung gleich der Leuchtkraftverteilung ist. Die Leuchtkraftverteilungen in der Milchstraße sind relativ flach. Der Exponent der kumulativen Verteilung ist -0.64 für HMXBs und -0.26 für LMXBs. Darüberhinaus zeigt die Leuchtkraftverteilung für LMXBs einen scharfen Abschluß bei einer Leuchtkraft von ca. $2.7 \cdot 10^{38} \mathrm{erg} / \mathrm{s}$.

Die Leuchtkraftverteilung erlaubt auch die Bestimmung der Gesamtleuchtkraft der Röntgendoppelsterne. Man erwartet, daß die Gesamtleuchtkraft von HMXBs mit der Sternentstehungsrate und die von LMXBs mit der stellaren Masse einer Galaxis skaliert. Daher können diese beiden Werte für die Milchstraße zur Kalibrierung dieser Relationen für andere Galaxien verwendet werden. Aufgrund der Flachheit der Leuchtkraftverteilungen wird die Gesamtleuchtkraft von relativ wenigen leuchtkräftigen Quellen dominiert. Die Gesamtleuchtkraft von Röntgendoppelsternen in der Milchstraße beträgt ca. $2-3$. $10^{39} \mathrm{erg} / \mathrm{s}$. LMXBs tragen dazu ungefähr $90 \%$ bei. Normiert auf die stellare Masse beziehungsweise die Sternentstehungsrate beträgt die Leuchtkraft von LMXBs ca. $5 \cdot 10^{28}$ $\operatorname{erg~s}^{-1} / \mathrm{M}_{\odot}$ und von HMXBs ca. $5 \cdot 10^{37} \mathrm{erg} \mathrm{s}^{-1} /\left(\mathrm{M}_{\odot} / \mathrm{a}\right)$. Da diese Quellen stark variabel auf Zeitskalen von Sekunden bis Jahren sind, ist auch die Gesamtleuchtkraft stark variabel auf diesen Zeitskalen.

Der zweite Teil der Arbeit beschäftigt sich mit dem Zusammenhang zwischen der Population von HMXBs und der Sternentstehungsrate in Galaxien.

Wie oben erwähnt, erwartet man, daß die Population von HMXBs mit der Sternentstehungsrate gekoppelt ist, da der optische Begleitstern eine niedrige Lebenserwartung hat. Aufgrund der niedrigen Lebenserwartung werden HMXBs sehr kurz nach der Entstehung der Systeme zu beobachten sein und nach einer relativ kurzen Zeit von einigen Jahrmillionen wieder verschwinden, wenn der Sekundärstern ebenfalls in einer Supernova explodiert.

Die einfachste Annahme ist, daß die Zahl der HMXBs proportional zur Sternentstehungsrate der letzten wenigen Jahrmillionen ist. Dies wird durch eine Normierung der Leuchtkraftfunktion ausgedrückt, die proportional zur Sternentstehungsrate ist.

Um diesen Zusammenhang zu untersuchen, habe ich die Leuchtkraftfunktionen mehrerer Galaxien, die mit dem Chandra-Röntgenteleskop beobachtet worden sind, verwendet. Aufgrund der exzellenten Aufösung können Leuchtkraftfunktionen für diese Galaxien konstruiert werden, solange die Galaxis nicht weiter als 20-30 Mpc entfernt ist. Außerdem habe ich auch Daten weit entfernter Galaxien, hauptsächlich aus dem sogenannten Hubble Deep Field, verwendet, um zu untersuchen, ob die von lokalen Galaxien gewonnene Relation für die Gesamtleuchtkraft und Sternentstehungsrate auch bei höheren Rotverschiebungen gültig ist.

Die Untersuchung von Galaxien in diesem Zusammenhang, unterliegt hauptsächlich zwei Einschränkungen. In nahe liegenden Galaxien kann man nicht zwischen LMXBs und HMXBs unterscheiden. Da LMXBs aber nicht mit der aktuellen Sternentstehungsrate korrelieren, sind sie als Untergrund zu betrachten. Dieses Problem kann gelöst werden, indem lediglich Galaxien mit einem ausreichend hohen Verhältnis von Sternentstehungsrate 
zu stellarer Masse verwendet werden. Die stellare Masse ist dabei ein Maß für den Beitrag von LMXBs zur Röntgendoppelstern-Population einer Galaxis. Ein aktiver Galaxienkern, der die Emission von Röntgendoppelsternen leicht übertreffen kann, ist in diesen Galaxien kein Problem, da es aufgrund der Auflösung von Chandra möglich ist, diese Quelle aus der Leuchtkraftfunktion zu eliminieren.

In Galaxien bei hohen Rotverschiebungen ist ein aktiver Kern ein Problem, daß mit Hilfe anderer Diagnostikmethoden wie optischen Spektren eliminiert werden kann.

Die Untersuchung der verschiedenen Leuchtkraftverteilungen der verwendeten Galaxien ergibt, daß die Form der Leuchtkraftverteilung für alle Galaxien gleich ist und lediglich die Normierung sich proportional zur Sternentstehungsrate ändert. Die allgemeine Form der Leuchtkraftfunktion ist eine Exponentialfunktion in der Leuchtkraft mit einem Exponenten von -0.61 in der kumulativen Form, einer Normierung direkt proportional zur Sternentstehungsrate und einem scharfen Abschluß bei ca. $2 \cdot 10^{40} \mathrm{erg} / \mathrm{s}$.

Die Universalität der Leuchtkraftfunktion ergibt, daß die Anzahl und Gesamtleuchtkraft von HMXBs ein guter Indikator für die Sternentstehungsrate in Galaxien ist.

Betrachtet man den Zusammenhang von Gesamtleuchtkraft und Sternentstehungsrate, so zerfällt dieser in zwei Bereiche, einen nicht-linearen bei niedrigen Sternentstehungsraten unter ca. 4-5 $\mathrm{M}_{\odot} / \mathrm{a}$ und einen linearen Teil bei höheren Sternentstehungsraten. Diese Zweiteilung ergibt sich aus einem statistischen Effekt der Form der Leuchtkraftfunktion, der in Kapitel 6.8 diskutiert wird. Die Existenz des linearen Teils ist darüberhinaus eine Bestätigung für die Existenz einer maximalen Leuchtkraft in der Leuchtkraftfunktion bei ca. $2 \cdot 10^{40} \mathrm{erg} / \mathrm{s}$. Beobachtungen des Hubble Deep Field-North ergeben, daß der ermittelte Zusammenhang zwischen Gesamtleuchtkraft und Sternentstehungsrate auch bei hohen Rotverschiebungen gültig ist. Das heißt auch, daß die Entstehung von Röntgendoppelsternen bei hohen Rotverschiebungen ähnlich verlaufen muß wie im lokalen Universum.

Teile der vorliegenden Arbeit wurden in den Artikeln Grimm et al. (2002) und Grimm et al. (2003) veröffentlicht. 


\section{Introduction}

\subsection{X-ray binaries}

$\mathrm{X}$-ray binaries were among the first objects detected, in the form of the source Sco X1 , the brightest X-ray source in the sky, when the first X-ray missions were launched to space (Giacconi et al. 1962). They are the brightest compact sources in the medium X-ray range $(2-10 \mathrm{keV})$. The nature of these sources was recognised only shortly later (Shklovsky 1967; Prendergast \& Burbidge 1968). Since then a large number of satellite missions, from UHURU, Ariel V, Ginga, EXOSAT to ROSAT and many other spacecraft have greatly extended our knowledge about the X-ray and physical properties of X-ray binaries.

$\mathrm{X}$-ray binaries are objects that, due to their peculiar nature, are of interest for very different branches of physics and astronomy. An X-ray binary consists of a compact object, either a neutron star or a black hole called the primary, and a companion star from which the compact object accretes mass. The companion, or secondary, can be any stellar object, from a brown dwarf (e.g. Bildsten \& Chakrabarty (2001)) to a main sequence star to a degenerate white dwarf (e.g. Tutukov et al. (1987)). The special conditions necessary to make these systems result in a rather small number in a given galaxy, despite most stars being in binary or multiple systems (Illarionov \& Sunyaev 1975). The systems have to have parameters that allow mass transfer to occur from the secondary to the primary in a more or less stable way. That means the system has to be separate in order not to merge in a common envelope phase, but also close enough after the supernova explosion of the primary to allow mass transfer. Since these parameters depend on the masses of the objects, on the orbital parameters, and on the evolutionary status of the secondary, and in general the history of the system, the conditions for a more or less stable mass transfer are fulfilled only for very few systems. Population synthesis models of binaries predict of order a few thousand systems in total in the Milky Way, see e.g. Dalton \& Sarazin (1995); Icko Iben et al. (1995).

Also in practice white dwarfs can also be the primary object in an accreting binary system. If the white dwarf is accreting this type of system is called cataclysmic variable $(\mathrm{CV})$. The end result of such a system might be a supernova type Ia. However due to the rather small mass and large size of the white dwarf, its potential well is relatively more shallow than for neutron stars and black holes. Therefore most of the emitted radiation from the accreted material is released in the optical and UV, with only a minor fraction released in X-rays. For that reason they are not generally referred to as X-ray binaries.

Despite their relatively low number, X-ray binaries have been classified in various ways, depending on the property of interest. The sum of diverse companion stars, orbital param- 
eters, different modes of accretion, and the resulting multitude of X-ray properties have led to a zoo of nomenclature for different types of X-ray binaries that are classified either by their secondary, e.g. Be X-ray binaries, or by their activity, e.g. soft X-ray transients or X-ray pulsars. A more thorough description of the nomenclature and references about the nature and multitude of X-ray binary systems can be found e.g. in Lewin et al. (1994).

In this dissertation the most important distinction is the mass of the secondary. In general, X-ray binaries are divided in two or three different classes, depending on the mass of the secondary. There is the class of low mass X-ray binaries (LMXBs) where the optical companion has a mass, $\mathrm{M}_{2}$, below $\sim 1 \mathrm{M}_{\odot}$. At the other extreme are high mass X-ray binaries (HMXBs) with a companion mass above $\sim 5-10 \mathrm{M}_{\odot}$. In between are so-called intermediate mass X-ray binaries (IMXBs) in which the companion lies between $\sim 1-5$ $\mathrm{M}_{\odot}$. This last class is only sparsely populated in the Milky Way.

This distinction is not just an arbitrary categorisation. To obtain a neutron star or black hole the star, whose remnant is the primary object, must be more massive than $\sim 8 \mathrm{M}_{\odot}$. This entails a very short lifetime since the time spent on the main sequence is roughly proportional to $\mathrm{M}^{-2}$. Thus the lifetime of the progenitor of the primary is very short for all possible masses of the primary progenitor, and of the order a few million years. The mass of the secondary on the other hand defines the future of the X-ray binary system in a stronger way than the mass of the primary, or the predecessor of the primary. Due to the steep dependence of the main sequence lifetime on the mass, however, the evolutionary timescale of stars below $1 \mathrm{M}_{\odot}$ and stars above $10 \mathrm{M}_{\odot}$ is different by a factor of $\sim 100$. These strongly differing timescales are of great importance for the understanding of formation processes of X-ray binaries. Due to their short lifetime HMXBs are good indicators of recent star forming activity in galaxies. To investigate this relation, as it will be discussed in chapter 6, it is helpful to know about the HMXB population in the Milky Way.

HMXBs were first recognised as short-lived objects fed by the gas supply of a massive star as a result of the discovery of Cen X-3 as an X-ray pulsar by UHURU. The X-ray source is in a binary system with an optical companion of more than $17 \mathrm{M}_{\odot}$ (Schreier et al. 1972). The localisation and mass estimate of the $\mathrm{Cyg} \mathrm{X}-1 \mathrm{BH}$ was obtained from observations of a soft/hard state transition occurring simultaneously with a radio flare (Tananbaum et al. 1972), and following optical observations of a bright massive counterpart (Bolton 1972; Lyutyi et al. 1973). Dynamics of interacting galaxies, e.g. Antennae, provide an additional upper limit on the evolution and existence time scale of HMXBs since the tidal tails and wave patterns in which star formation is most vigorous are very short-lived phenomena, of the order of a crossing time of interacting galaxies (Toomre \& Toomre 1972; Eneev et al. 1973).

Another classification scheme for X-ray binaries is connected with the nature of the compact object. This classification encompasses black hole X-ray binaries and neutron star X-ray binaries. From an observational point of view the main differences between the different categories are the mass of the compact object and the existence or lack of a hard surface. For neutron stars the canonical mass is $\sim 1.4 \mathrm{M}_{\odot}$, the so-called Chandrasekhar mass, but, due to uncertainties in the equation of state of neutron stars, compact objects between 1.4 and $3 \mathrm{M}_{\odot}$ are labelled as neutron stars. $3.3 \mathrm{M}_{\odot}$ is the maximum mass for a 
neutron star with a stiff equation of state to be stable against further gravitational collapse (Stergioulas 1998). Consequently compact objects with a mass above $3 \mathrm{M}_{\odot}$ are believed to be black holes.

To estimate the mass of a compact object in a binary system optical observations, i.e. spectroscopy, are necessary. These estimates for black hole and neutron star masses reveal an apparent gap between the average mass of a neutron star, around $1.4 \mathrm{M}_{\odot}$, and the masses of black holes which are above $\sim 4 \mathrm{M}_{\odot}$ (Orosz 2002). This mass separation would make the distinction between black holes and neutron stars easier if confirmed by more precise data.

The observational differences between black hole and neutron star binaries are more subtle than in the case of the optical companion and also less certain. Nevertheless there is a trend that black hole X-ray binaries generally have softer X-ray spectra than neutron star binaries, which might be related to the fact that on the one hand a neutron stars have in general a strong magnetic field and a hard surface whereas black holes on the other hand have no magnetic field and an event horizon. Moreover there is strong indication that the variability of the X-ray emission from neutron stars extends to higher frequencies than in black hole systems, also likely due to the presence of a hard surface (Sunyaev \& Revnivtsev 2000). For a neutron star half of the energy release takes place on the surface of the neutron star. The resulting plasma column on top of the surface may be the origin of variability and oscillations, so-called kilo-Hertz quasi periodic oscillations ( $\mathrm{kHz} \mathrm{QPO}$ ), that have no counterpart in black holes accretion where matter simply traverses the event horizon without any interaction.

\subsection{Accretion}

The processes by which X-ray binaries become observable for us are related to the accretion of matter from the secondary onto the compact object. The theory of accretion goes back to Bondi \& Hoyle (1944) who dealt with spherical accretion of gas onto a compact object. However, this kind of accretion does not release sufficient amounts of energy to explain the most luminous sources observed in the Galaxy and, moreover, does not account for angular momentum of the accreting matter which in most astrophysically interesting scenarios is important. In terms of accretion of matter with angular momentum Shakura \& Sunyaev (1973) described a geometrically thin, optically thick disk of gas that is either gas pressure or, in the inner parts, radiation pressure dominated and has an anomalous viscosity that is assumed to be connected with the magnetic field of the accreting matter. This viscosity allows matter to transfer angular momentum outwards and thus accrete onto the compact object. The energy released during this process is emitted from the surface of the disk, and is emitted at many wavelengths. Because the disk temperature is inversely dependent on the radius, X-rays are emitted predominantly from the inner regions, where the disk is hottest.

This model has been refined and modified to include other processes and different boundary conditions. An important modification, for example, is the accretion onto a strongly 
magnetised neutron star. If the magnetic field is strong enough, roughly above $10^{10}-10^{11}$ Gauss, it disrupts the flow of the accretion disk and diverts the flow along the magnetic field lines to the magnetic poles of the neutron star. These objects can be observed as so-called X-ray pulsars (Truemper et al. 1978). The emission from these sources can be used to measure the magnetic field strength of the neutron star (Gnedin \& Suniaev 1974).

Other modifications involve the inclusion of a hot $(\sim 100 \mathrm{keV})$ plasma above the accretion disk, in the form of a corona or an atmosphere, that is responsible for emission of hard X-rays due to Comptonisation of photons emitted from the disk (Sunyaev \& Titarchuk 1985). A more complete and detailed discussion of accretion theory with references can be found in the book of Frank et al. (1992).

An important concept in the context of accretion is the so-called Eddington luminosity. The idea is that an object can accrete matter only as long as the gravitational attraction of the object is stronger than the radiation pressure produced by the emitted photons from infalling matter further in. If the radiation pressure becomes dominant the infalling material is dispersed and accretion ceases. The photon pressure works predominantly on the electrons in the plasma and the electric field that is subsequently generated pulls on the nuclei. Therefore the Eddington luminosity depends also on the chemical composition of the plasma. In the ideal case of hydrogen plasma the equilibrium state is described by the formula:

$$
\begin{aligned}
\vec{F}_{\text {grav }} & =-\vec{F}_{\text {rad }} \\
\frac{G M m}{r^{2}} & =\frac{q \sigma_{T}}{c} .
\end{aligned}
$$

In the spherically symmetric case

$$
q=\frac{L}{4 \pi r^{2}}
$$

and the Eddington luminosity,

$$
L_{E d d}=\frac{4 \pi G M m c}{\sigma_{T}},
$$

is independent of distance, where $G$ is the Newton's gravitational constant, $M$ the mass of the accreting object, $m$ the mass of the accreting particle, generally the proton, $r$ the distance from the object, $q$ the photon flux, $\sigma_{T}$ the cross section of the photon-particle interaction, generally taken to be the Thomson cross section, and $c$ the speed of light. The Eddington luminosity $L_{E d d}$ is therefore the maximum luminosity an object can emit. For example a $1.4 \mathrm{M}_{\odot}$ neutron star with the above mentioned parameters and solar chemical abundance has an Eddington luminosity of $\sim 2 \cdot 10^{38} \mathrm{erg} / \mathrm{s}$. Moreover, there are two modes of accretion that are mostly related to the nature of the secondary. Accretion can take the form of Roche lobe overflow, i.e. the secondary fills its Roche lobe, either due to evolutionary processes or due to shrinking of the Roche lobe. Or the compact object can accrete from the wind of the secondary. The latter mode is obviously only important for stars that have an appreciable mass loss rate due to a wind, which is only the case for stars more massive than $\sim 5 \mathrm{M}_{\odot}$. Therefore wind accretion takes place only in HMXBs, 
not LMXBs. For suitable orbital parameters the wind accretion rate can reach very high values since the mass loss rate of a massive star can also be very high, up to $\sim 10^{-4} \mathrm{M}_{\odot} / \mathrm{yr}$ for Wolf-Rayet stars. However, the accretion rate is also dependent on the capture radius, i.e. the radius at which the gravitational attraction is able to force the matter of the wind in a bound orbit. The capture radius is given by

$$
r_{\text {capture }}=\frac{2 G M}{v_{0}^{2}}
$$

where $G$ is Newton's gravitational constant, $M$ is the mass of the compact object and $v_{0}$ is the velocity of the wind at the orbit of the compact object. Matter within the capture radius is accreted, matter outside escapes the system. The capture radius is a function of the mass of the compact object and the wind velocity, which can be several thousand kilometres per second. Therefore wind accretion is more effective for black holes than for neutron stars.

On the other hand for systems accreting via Roche lobe overflow the mass of the compact object does not play such a crucial role. If a star fills its Roche lobe matter can flow freely from the surface of the star into the potential well of the compact object through the inner Lagrange point. Due to its non-zero angular momentum with respect to the compact object it forms an accretion disk around the compact object.

\subsection{Motivation and Outline}

This thesis can be divided into 2 parts that are both observationally motivated. The first part deals with the Galactic X-ray binary population. Despite being known as celestial objects for almost 40 years now, a detailed study of the Galactic X-ray binary population became possible only recently with the huge progress in obtaining optical identifications and distance determinations. This allows the study of the spatial distribution of different classes of Galactic X-ray binaries and thus the construction of luminosity functions. Moreover it leads to a picture of the appearance of the Galaxy in X-rays for an outside observer, like what we observe today with the CHANDRA (Weisskopf et al. 2000) and XMM-Newton (Jansen et al. 2001) X-ray observatories. A study of the X-ray binaries in the Milky Way also simplifies the investigation of X-ray binary populations in other galaxies, an example of which is presented in the second part of this thesis.

\subsubsection{Galactic X-ray binary population}

The combination of data from optical and IR observations and, foremost, the long time data from the all sky monitor of RXTE (Bradt et al. 1993) allow, for the first time, a more detailed study of the Galactic X-ray binary population. There have been attempts to study the the 3D spatial distribution of X-ray binaries by van Paradijs \& White (1995) and White \& van Paradijs (1996). However these studies suffered from the smaller number 
of sources and/or distances available. We present here the first comprehensive compilation of X-ray binaries with optical identifications and distance estimates.

Since its launch, the CHANDRA X-ray observatory has observed numerous galaxies of every type, from early-type ellipticals to late-type spirals and irregulars. A main discovery of these CHANDRA observations was the existence of numerous point-like sources with luminosities in the CHANDRA spectral band considerably higher than the Eddington luminosity of a $1.4 \mathrm{M}_{\odot}$ neutron star. Observations of nearby galaxies have a great advantage compared with observations of X-ray sources in our Galaxy: All objects observed in a particular galaxy are equidistant and therefore it is straightforward to construct the luminosity function. However, even with the angular resolution and sensitivity of CHANDRA we are restricted to nearby galaxies $(d \lesssim 20-30 \mathrm{Mpc})$ and we are able to observe only the high luminosity end of the luminosity function. Observations of compact sources inside our Galaxy thus open the unique possibility to construct a luminosity function in a much broader range of luminosities.

In the first part of this thesis, chapter $2-4$, we use data of the All-Sky Monitor (ASM) (Levine et al. 1996) aboard the Rossi X-ray Timing Explorer (Brandt et al. 1996) to investigate the following topics.

- Using ASM data we construct the $\log (\mathrm{N})-\log (\mathrm{S})$ relation of different classes of Galactic X-ray sources, chapter 2. This allows to draw some first conclusions about the X-ray binary population and, with help of other observations, lays the basis to study the spatial distribution of X-ray binaries in the Milky Way.

- Using distance determinations and optical identifications to a large subset of ASM sources we investigate the spatial distribution of HMXBs and LMXBs. Our analysis of ASM data permits us to show how our Galaxy would look from outside in different projections. This will allow us to compare data about our Galaxy with new CHANDRA observations, chapter 3.1. Our results confirm earlier theoretical expectations in terms of the spatial distribution of X-ray binaries, i.e. that LMXBs are concentrated in the central part of the Milky Way, whereas HMXBs only populate the disk of the Galaxy.

- Using ASM data, existing information about the source distances and a model of the mass distribution in the Milky Way we construct the luminosity function of HMXBs and LMXBs in our Galaxy, chapter 3. Since in nearby galaxies we observe the luminosity function directly, this allows a direct comparison of the Galactic luminosity functions with other galaxies.

- The distribution and the number of HMXBs are expected to reflect the rate of star formation trace its location. On the other hand, the luminosity of the LMXB component is expected to be proportional to the total mass of the old stellar population of the Milky Way. Therefore our Galaxy will become an important point in the future calibration curves of $L_{\mathrm{HMxB}} / S F R$ and $L_{\mathrm{LMXB}} / M_{\text {galaxy }}$, as discussed in chapter 3.2.2. 
- The few most luminous Galactic X-ray binaries practically dominate the X-ray luminosity of our Galaxy. At the same time the majority of the brightest X-ray binaries are extremely variable on all time scales from milliseconds to years-tens of years. Therefore the luminosity of our Galaxy as a whole would also be subject to strong variability, which may allow in the future to distinguish low-luminosity AGN from X-ray binary dominated galaxies, chapter 3.2.2.

- Our analysis of ASM data and data from other spacecraft shows that at least for 17 X-ray sources in our Galaxy, ASM or other spacecraft detected flux reaching or exceeding the level corresponding to the Eddington critical luminosity for a $1.4 \mathrm{M}_{\odot}$ neutron star, see Table 3.2.3. Maximal fluxes detected were up to 10 times higher than the Eddington value for a neutron star. In at least 7 sources the compact object has been identified as a neutron star based on the detection of X-ray pulsations or X-ray bursts, therefore we know with certainty that the peak luminosity exceeded the Eddington limit. Moreover, the total number of super-Eddington sources might be higher because we know from broad band observations that the bulk of the luminosity can be emitted outside the ASM sensitivity band, chapter 3.2.3.

\subsubsection{HMXB-SFR connection}

The second part of the thesis, chapter 5-6 is concerned with the connection of star formation rate (SFR) and the luminosity function of HMXBs. X-ray observations open a new way to determine the star formation rate in young very distant galaxies. CHANDRA observations of actively star forming galaxies in our vicinity and RXTE/ASM, ASCA, and MIR-KVANT/TTM data on HMXB populations in our Galaxy and the Magellanic Clouds provide a possibility to calibrate the dependence of SFR on the X-ray luminosity of a galaxy due to HMXBs. For nearby, spatially resolved galaxies for which CHANDRA is able to resolve individual X-ray binaries we also have the opportunity to calibrate the dependence of SFR on the total number of HMXB sources.

In the absence of a bright AGN, the X-ray emission of a galaxy is known to be dominated by the collective emission of its X-ray binary populations (see e.g. Fabbiano (1994)). As mentioned above, the difference in the mass of the secondary results in drastically different evolution time scales for low and high mass X-ray binaries and, hence, different relations between both their number and collective luminosity, and to the instantaneous star formation activity and the stellar content of the parent galaxy. In the case of a HMXB, having a high mass companion, generally $M_{\text {optical }}>10 \mathrm{M}_{\odot}$ (Verbunt \& van den Heuvel 1994), the characteristic time scale is at most the nuclear time scale of the optical companion which does not exceed $\sim 2 \cdot 10^{7}$ years. For an LMXB, however, $M_{\text {optical }}$ is generally less than $1 \mathrm{M}_{\odot}$, and the timescale is on the order of $\sim 10^{10}$ years.

The prompt evolution of HMXBs makes them a potentially good tracer of the very recent star formation activity in a galaxy (Sunyaev et al. 1978) whereas, due to slow evolution, LMXBs display no direct connection to the present value of SFR. LMXBs rather are connected to the total stellar content of a galaxy determined by the sequence of star 
formation episodes experienced by a galaxy during its lifetime (Ghosh \& White 2001; Ptak et al. 2001; Grimm et al. 2002).

Several calibration methods are employed to obtain SFRs using UV, FIR, and radio flux from distant galaxies. Many of these methods rely on a number of assumptions about the environment in the galaxy and suffer from various uncertainties, e.g. the influence of dust, escape fraction of photons, supernova relation to SFR, or the shape of the initial mass function (IMF). An additional and independent calibrator might therefore become a useful method for the determination of SFR. Such a method, based on the X-ray emission of a galaxy, might circumvent one of the main sources of uncertainty of conventional SFR indicators - absorption by dust and gas. Indeed, galaxies are mostly transparent to X-rays above about $2 \mathrm{keV}$, except for the densest parts of the most massive molecular clouds.

The existence of various correlations between X-ray and optical/far-infrared properties of galaxies has been noted and studied in the past. Based on Einstein observations of normal and star-burst galaxies from the IRAS Bright Galaxy Sample, Griffiths \& Padovani (1990) and David et al. (1992) found correlations between the soft X-ray luminosity of a galaxy and its far-infrared and blue luminosity. Due to the limited energy range $(0.5-3 \mathrm{keV})$ of the Einstein observatory data one of the main obstacles in quantifying and interpreting these correlations was proper accounting for absorption effects in soft X-rays and intrinsic spectra of the galaxies which resulted in considerable spread in the derived power law indices of the $\mathrm{X}$-ray - FIR correlations, $\sim 0.7-1.0$. Moreover, supernova remnants are bright in the soft band of the Einstein observatory. CHANDRA, however, is able to distinguish SNRs from other sources due to its sensitivity to harder X-rays. Although the X-ray data were not sufficient to discriminate between contributions of different classes of X-ray sources, David et al. (1992) suggested that the existence of such correlations could be understood with a two component model for X-ray and far-infrared emission from spiral galaxies, consisting of old and young populations of the objects having different relations to the current star formation activity in a galaxy. The uncertainty related to absorption effects in soft Xrays was recently eliminated by Ranalli et al. (2003), who extended these studies to the harder energy band of 2-10 keV based on BeppoSAX and ASCA data. In particular, they found a linear correlation between total X-ray luminosity of a galaxy and both radio and far-infrared luminosities and suggested that the X-ray emission might be directly related to the current star formation rate in a galaxy and that such a relation might also hold at higher redshifts.

One of the main results presented here in the context of the luminosity-SFR relation is that in the low SFR regime the relation between SFR and collective luminosity of HMXBs is non-linear, $L_{X} \propto \mathrm{SFR}^{\sim 1.7}$, and becomes linear only for sufficiently high star formation rates, when the total number of HMXB sources becomes sufficiently large. The non-linear $L_{X}-S F R$ dependence is caused by the fact that we measure the collective luminosity, which strongly depends on the brightest objects, of a population of discrete objects. This effect is discussed in detail in chapter 6.8.

There are, however, two main obstacles to use the X-ray luminosity of a galaxy as a SFR indicator. Firstly, if an active nucleus (AGN) is present in a galaxy it can easily outshine HMXBs in X-rays. In principle, the presence of an AGN component might be 
identified and, in some cases separated, due to the distinctive X-ray spectra of AGN and $\mathrm{X}$-ray binaries, provided the energy coverage is sufficiently broad. Secondly, there is the dichotomy into LMXBs and HMXBs, which both have somewhat similar spectra, that also could probably be distinguished provided sufficiently broad band coverage and sufficient signal-to-noise ratio. To estimate the SFR one is interested only in the luminosity of HMXBs, therefore the LMXB contribution needs to be subtracted. This could, in principle, be done based on an estimate of the stellar mass of a galaxy. The results of the study of the X-ray binary population of our Milky Way, chapters 2-4, and knowledge of the Galactic SFR allow to estimate at which point the emission of HMXBs dominates the emission of a galaxy. A threshold which obviously depends on the ratio of SFR to stellar mass of a galaxy. We found, that roughly at a ratio of $\sim 0.05 \mathrm{M}_{\odot} /$ yr per $10^{10} \mathrm{M}_{\odot}$ of total dynamical mass, or $\sim 0.5 \mathrm{M}_{\odot} /$ yr per $10^{10} \mathrm{M}_{\odot}$ of stellar mass, the emission of HMXB sources begins to dominate the X-ray emission of a galaxy (where the SFR value refers to a formation rate of stars more massive than $\sim 5 \mathrm{M}_{\odot}$ ). It should be emphasised, however, that even in the worst case the X-ray luminosity based SFR estimate should be able to provide an upper limit on the ongoing star formation activity in a galaxy.

Future observations with CHANDRA and XMM-Newton, and upcoming X-ray missions, such as Astro-E, Constellation-X and XEUS, the last having 1 arc sec angular resolution and a 100 times larger effective area than CHANDRA, will permit us to derive the SFR of galaxies from X-rays even at high redshifts. We know from optical and radio data that the SFR was much higher in galaxies at $\mathrm{z} \sim 2-5$ (Madau \& Pozzetti 2000). Therefore we could expect that in these galaxies the contribution of HMXBs was strongly exceeding the contribution of LMXBs. 
1. Introduction 
Part I.

The Milky Way 



\section{Milky Way $\log (\mathrm{N})-\log (\mathrm{S})$}

\subsection{RXTE All-Sky Monitor Data}

In order to construct the $\log (\mathrm{N})-\log (\mathrm{S})$ distributions and luminosity functions we used the publicly available data of ASM. The ASM instrument is sensitive in the 2-10 keV energy band which is divided into 3 broad energy channels and provides $80 \%$ sky coverage for every satellite orbit ( $\sim 90$ minutes). Due to its all-sky nature and long operational time, $\sim 5$ years, the ASM instrument is ideally suited for studying time averaged properties of sources. The light curves are obtained by RXTE GOF (Levine et al. 1996) for a preselected set of sources from the ASM catalogue. The catalogue consists of sources which have reached an intensity of more than 5 mCrab at any time (Lochner \& Remillard 1997), and as of June 2000 included 340 sources of which 217 are galactic and 112 extragalactic, and 10 unidentified. The distribution of galactic sources on the sky is shown in Fig. 2.1. For a detailed description of selection criteria and a list of sources see Lochner \& Remillard (1997). The 1 day sensitivity of ASM is $\approx 10$ mCrab corresponding to a count rate of $0.75 \mathrm{cts} \mathrm{s}^{-1}$. The ASM count rate has been converted to energy flux assuming a Crab-like spectrum and using the observed Crab count rate:

$$
F\left[\mathrm{erg} \mathrm{s}^{-1} \mathrm{~cm}^{-2}\right]=3.2 \cdot 10^{-10} \cdot R\left[\operatorname{cts~s}^{-1}\right] .
$$

The 1-dwell ASM light curves have been retrieved from the RXTE public archive ${ }^{1}$ at HEASARC and cover a time period from the start of the mission through 27/04/00. In order to construct $\log (\mathrm{N})-\log (\mathrm{S})$ the light curves have been averaged over the entire period of available data which might differ for different sources. We did not account in any way for orbital variations or eclipses, as e.g. in Cen X-3. Important for the analysis presented below are the questions of systematic errors in the light curves and of the completeness limit of the ASM catalogue.

\subsubsection{Systematic errors}

The ASM light curves are assumed to have a systematic error at the level of $\sim 3 \%$ which is added in quadrature to the statistical errors in the light curves provided by the RXTE GOF. The systematic error has been estimated using Crab data and refers to the $\sim$ dwellday time scales. The formal errors for the average fluxes calculated from the entire ASM light curves are very small $\sim 0.1-0.2 \mathrm{mCrab}\left(\sim 1-2 \cdot 10^{-2}\right.$ cts s $\left.^{-1}\right)$. In the presence

\footnotetext{
${ }^{1} \mathrm{ftp}$ ///legacy.gsfc.nasa.gov/xte/data/archive/ASMProducts/definitive_1dwell/
} 


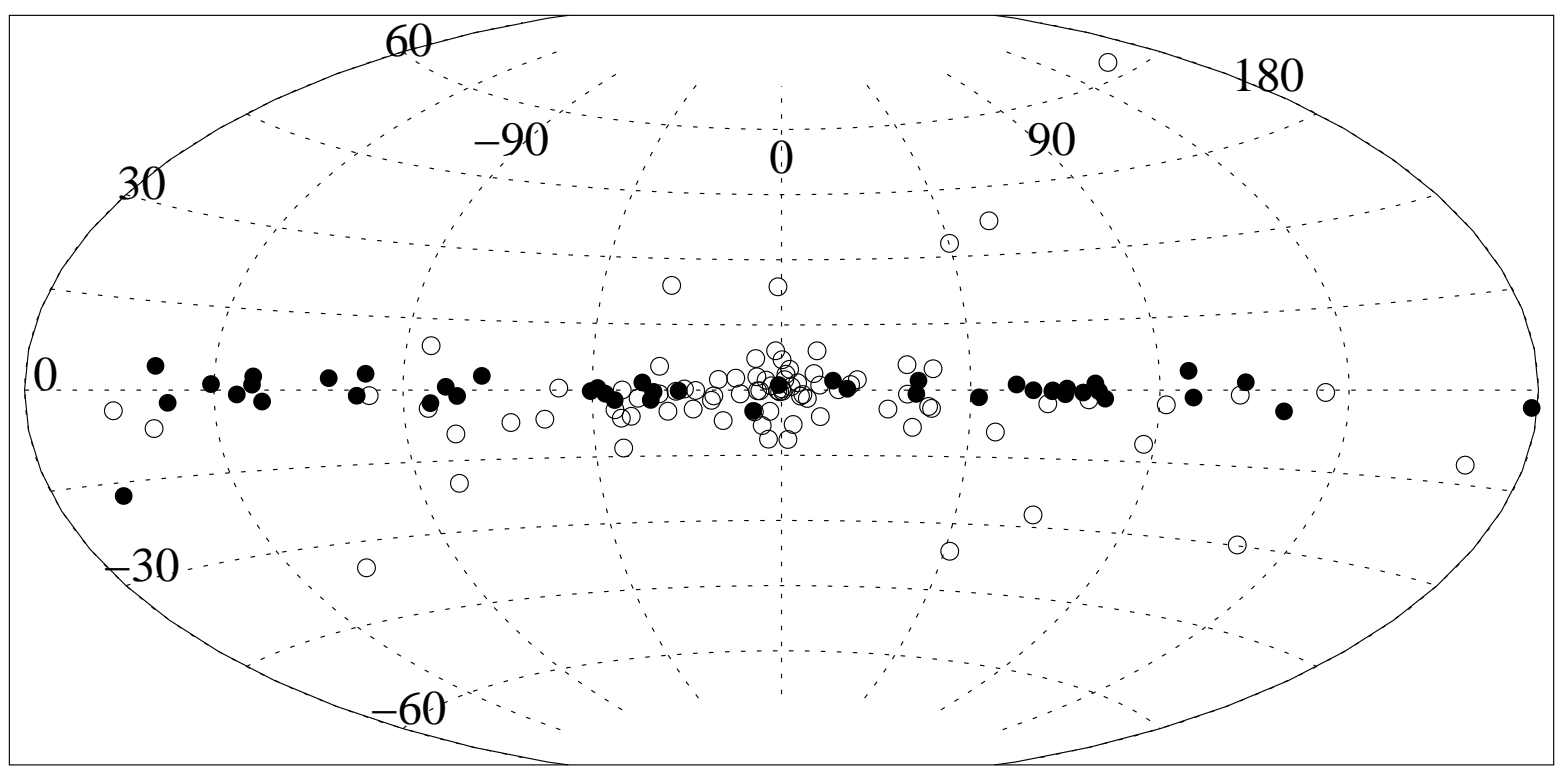

Figure 2.1.: Distribution of LMXBs (open circles) and HMXBs (filled circles) in the Galaxy. In total 86 LMXBs and 52 HMXBs are shown. Note the significant concentration of HMXBs towards the Galactic Plane and the clustering of LMXBs in the Galactic Bulge.

of systematic errors this might not correctly characterise the accuracy of the average flux estimate, especially for weak sources. The contribution of systematic errors to the average flux estimate depends on their statistical properties, in particular their correlation time scale. In order to investigate these properties we selected several sources believed to have constant X-ray flux, like SNRs or rotation powered pulsars, see Table 2.1, and rebinned their light curves with different bin durations ranging from 1 to 200 days.

\begin{tabular}{|l|cc|}
\hline Source & $\begin{array}{c}\text { average flux } \\
{\left[\text { cts s }^{-1} \text { ] }\right.}\end{array}$ & $\begin{array}{c}\text { excess RMS } \\
\text { [cts s }^{-1} \text { ] }\end{array}$ \\
\hline Cas A & $4.9 \pm 0.007$ & $\sim 0.08$ \\
Tycho SNR & $1.3 \pm 0.007$ & $\sim 0.04$ \\
Puppis A & $0.84 \pm 0.008$ & $\sim 0.05$ \\
Vela pulsar & $0.75 \pm 0.008$ & $\sim 0.01$ \\
CTB 33 & $0.35 \pm 0.014$ & $\sim 0.07$ \\
PSR 1259-63 & $0.18 \pm 0.012$ & $\sim 0.01$ \\
NGC 2024 & $0.09 \pm 0.008$ & $\sim 0.02$ \\
PSR J1713+0747 & $0.07 \pm 0.015$ & $\sim 0.01$ \\
PSR 1957+20 & $0.06 \pm 0.012$ & $\sim 0.02$ \\
XTE J1906+090 & $0.04 \pm 0.011$ & $\sim 0.03$ \\
\hline
\end{tabular}

TABLE 2.1.: List of sources used to estimate systematic errors.

${ }^{(a)}$ the errors are formally calculated using the errors in the light curves.

${ }^{(b)}$ upper limit on the unaccounted contribution of the systematic errors to the averaged flux, estimated from Fig. 2.2 . 


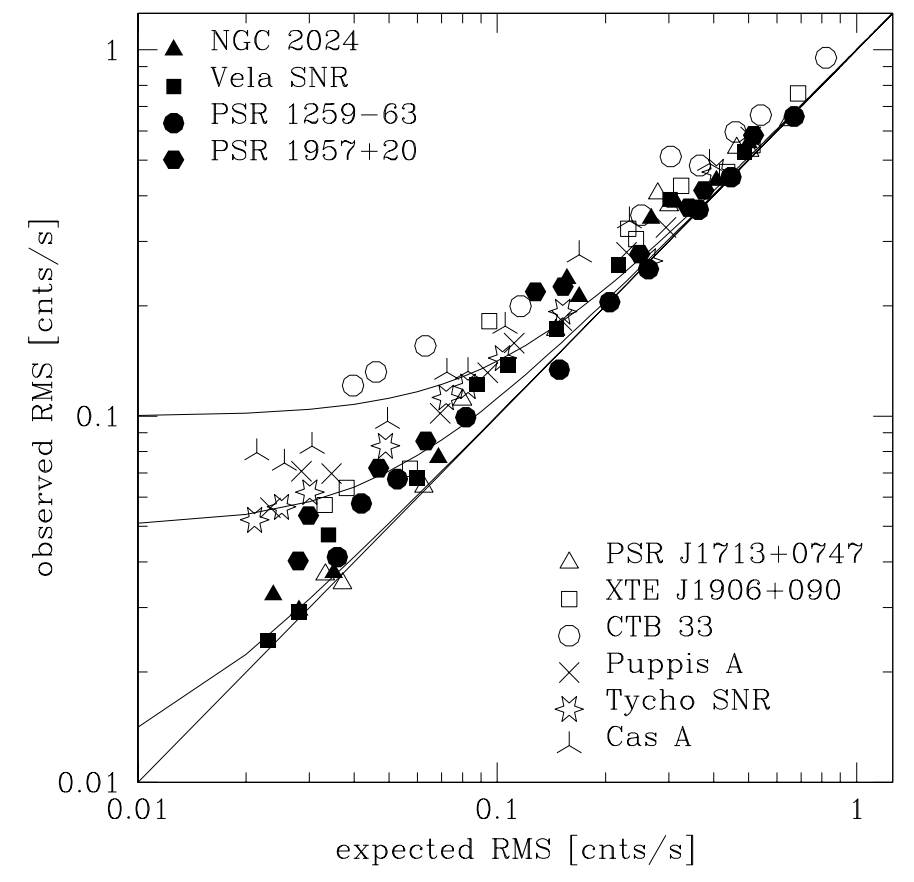

Figure 2.2.: Observed versus expected RMS for 10 different sources and for different time binnings. The bin duration varies from dwell time scale, i.e. $\sim 90$ seconds (upper right corner), to 200 days (lower left corner). Although there is considerable spread, the observed RMS is generally higher than expected, especially at large bin durations exceeding 50 days (expected RMS $<0.1 \mathrm{cts} \mathrm{s}^{-1}$ ). Assuming that systematic and statistical errors are independent the systematic error may be added to the statistical error in quadrature. This is shown by the solid curves for three different values of the systematic error: $0.01,0.05$ and $0.1 \mathrm{cts} \mathrm{s}^{-1}$.

For each binned light curve we computed the expected RMS from the errors given with the light curves and compared it with the observed RMS. The results are shown in Fig. 2.2. Ideally there should be a one-to-one correspondence between expected and observed RMS (straight line in Fig. 2.2). As can be seen from Fig. 2.2 this is not the case. The observed RMS somewhat exceeds the expected value, the discrepancy increasing towards large bin durations ( 50-200 days). The excess variance at large bin durations (lower-left part in Fig. 2.2) gives an upper limit on the unaccounted systematic error in the averaged flux estimate. As can be seen from Fig. 2.2 the particular value of the systematic error, though varying from source to source, is in the range of $0.01-0.1 \mathrm{cts} \mathrm{s}^{-1}$. We assumed a value of $0.05 \mathrm{cts} \mathrm{s}^{-1}$ (to be added in quadrature to the statistical error). We further verified that our conclusions are not sensitive to the value of the systematic error.

For 15 sources we obtain statistically significant, $\geq 3 \sigma$, negative average count rates. The majority of these sources, namely 14, are located in the Small and Large Magellanic Cloud and their negative average flux is apparently caused by source interference in these crowded regions. The remaining source also appears to suffer from interference with nearby sources. In particular, we have noticed that some of the light curves show a clear drop below zero count rate coincident in time with addition of new sources located nearby to the ASM catalogue. All these sources are excluded from our analysis.

\subsubsection{Completeness}

Important for the analysis presented below are two aspects of completeness: 


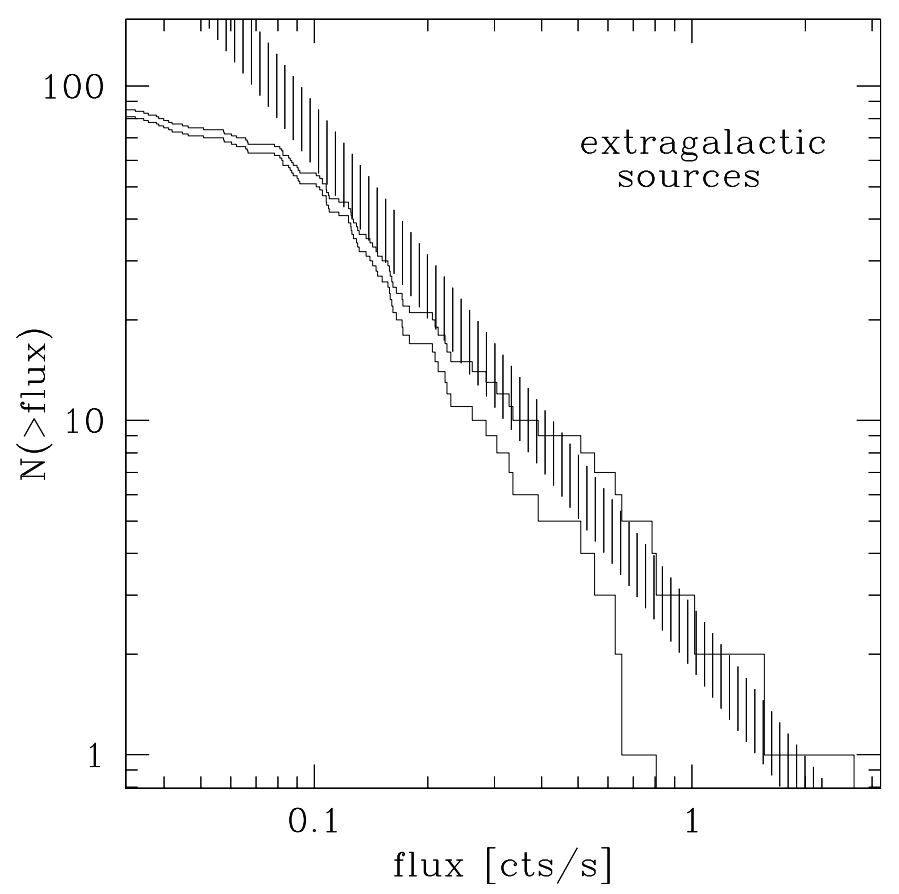

Figure 2.3.: $\log (\mathrm{N})-\log (\mathrm{S})$ distribution of extragalactic sources. Magellanic Cloud sources have been omitted. The upper histogram contains all extragalactic sources, the lower histogram excludes 4 nearby galaxy clusters (Perseus, Virgo/M 87, Coma and Centaurus). The shaded region shows the $\log (\mathrm{N})-\log (\mathrm{S})$ obtained by HEAO-1 A-2 for high latitude $\left(|b|>20^{\circ}\right)$ sources (Piccinotti et al. 1982). The width of the shaded region roughly accounts for the uncertainty of the RXTE/ASM and HEAO-1 A-2 calibration.

1. completeness flux limit of the ASM sample of the X-ray sources

2. completeness of the sample of galactic X-ray binaries which are optically identified and for which distance measurements are available

The first problem arises for example in studying $\log (\mathrm{N})-\log (\mathrm{S})$ distribution of all galactic sources and is addressed below. The second problem is important in analysing $\log (\mathrm{N})-$ $\log (\mathrm{S})$ distributions of various types of galactic X-ray sources and especially their luminosity functions. It is discussed in chapter 3.1.

Due to the present method of construction of the ASM catalogue its completeness limit is difficult to assess in any straightforward way. By definition the ASM sample includes all sources, galactic and extragalactic, which have reached an intensity of 5 mCrab at any time, which corresponds to a completeness limit of $\sim 0.37 \mathrm{cts} \mathrm{s}^{-1}$. On the other hand we know from the same ASM light curves that non-transient Galactic X-ray binaries have typical values of the ratio of maximum flux (on the time scale of dwell- $\sim$ day) to average flux of the order of few. Therefore, in terms of long term average values the ASM catalogue might be complete down to lower fluxes.

In order to indirectly probe the completeness limit of the ASM sample we use the fact that the $\log (\mathrm{N})-\log (\mathrm{S})$ relation for extragalactic sources is well known and follows a power law with index $-3 / 2$ (Forman et al. 1978), down to $\sim 3.8 \cdot 10^{-14} \mathrm{erg} \mathrm{s}^{-1} \mathrm{~cm}^{-2}$ (Ogasaka et al. 1998) which corresponds to ASM count rate of $1.2 \cdot 10^{-4}$ cts s $^{-1}$. The $\log (\mathrm{N})-$ $\log (\mathrm{S})$ relation for extragalactic sources based on ASM data is compared with HEAO A-1 
and ASCA results in Fig. 2.3. One can see that flattening of the source counts caused by incompleteness of the sample begins at a count rate of $\sim 0.1 \mathrm{cts} \mathrm{s}^{-1}$.

Therefore we set, somewhat arbitrarily, the completeness limit of the ASM sample of the X-ray sources at $0.2 \mathrm{cts} \mathrm{s}^{-1}$. We verified that our conclusions are not sensitive to the exact value.

\subsection{The $\log (\mathrm{N})-\log (\mathrm{S})$ distributions}

In order to calculate the number-flux relations the ASM light curves were averaged over the entire time span of available data for each source. The resulting $\log (\mathrm{N})-\log (\mathrm{S})$ relation for galactic sources is shown in Fig. 2.4. The differentiation between galactic and extragalactic sources was done using SIMBAD database. The overall shape and normalisation of the $\log (\mathrm{N})-\log (\mathrm{S})$ relation of Galactic sources is similar to that obtained by UHURU (Forman et al. 1978) and ARIEL V (Warwick et al. 1981). The UHURU result (Matilsky et al. $1973)$ is schematically shown in Fig. 2.4 by the solid line. The $\log (\mathrm{N})-\log (\mathrm{S})$ relation for different types of Galactic sources is also shown in Fig. 2.4.

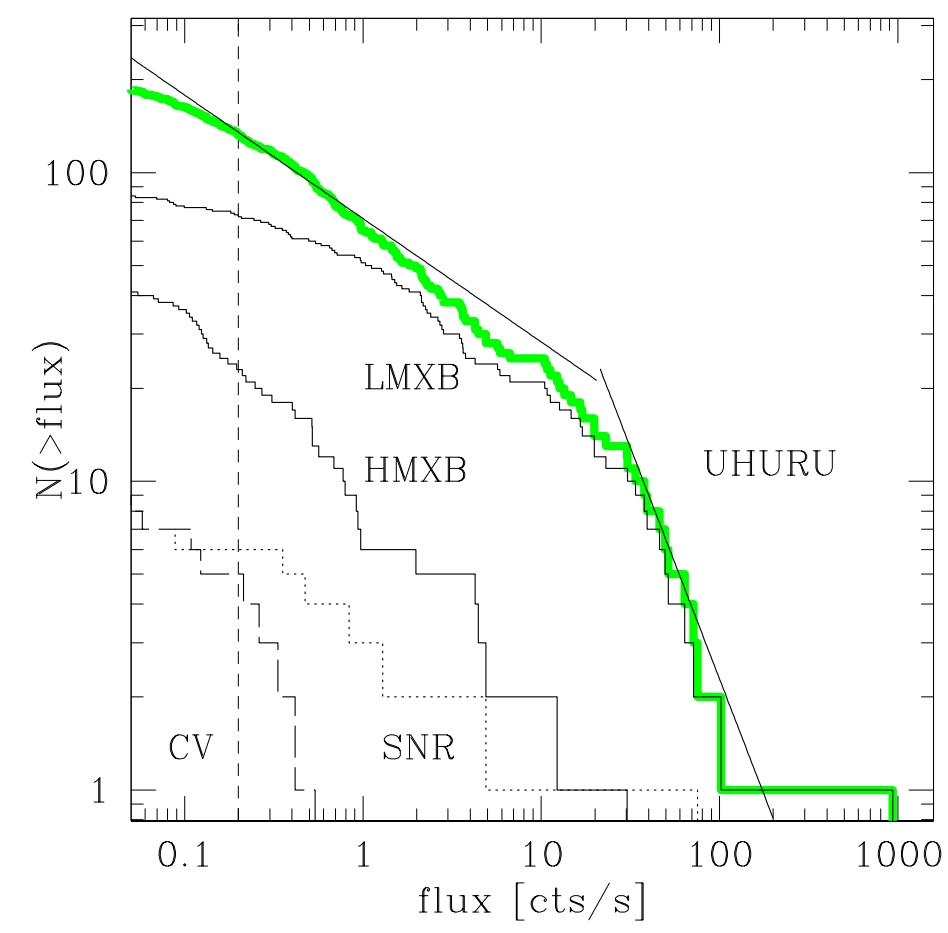

Figure 2.4.: Number-flux relation for all galactic sources derived from the entire ASM sample. The broken solid line shows schematically the number-flux relation for the low-latitude $|b|<$ $20^{\circ}$ sources obtained by UHURU (Matilsky et al. 1973). The vertical dashed line shows approximate completeness limit of the ASM sample. The thick grey histogram shows the $\log (\mathrm{N})-\log (\mathrm{S})$ for all Galactic sources observed by ASM. The four lower histograms show the contributions of different classes of sources to the total galactic $\log (\mathrm{N})-\log (\mathrm{S})$.

We further selected X-ray binaries from the sample and divided them into low mass (LMXB) and high mass (HMXB) binaries according to the mass of the optical companion, using the mass of the secondary, $\mathrm{M}_{2}$, of $2.5 \mathrm{M}_{\odot}$ to separate high and low mass systems. The precise value of this boundary affects classification of only few X-ray binaries (Her 
X-1, GX 1+4, GRO J1655-40 etc.). In doing so we used SIMBAD database, the Catalogue of X-ray Binaries (van Paradijs 1994), the Catalogue of CV, LMXB and related objects (Ritter \& Kolb 1998), the catalogues of low-mass X-ray binaries (Liu et al. 2001) and high-mass X-ray binaries (Liu et al. 2000) and in some cases publications on individual sources. Recently the donor star in GRS $1915+105$ was identified to be a K or M giant (Greiner et al. 2001) so this source is classified as an LMXB. Of 115 galactic X-ray binaries with average ASM flux exceeding our completeness limit of $0.2 \mathrm{cts} \mathrm{s}^{-1}$ only 6 sources were left unclassified. The fraction of unclassified sources is $\sim 5 \%$ and they have fluxes in the $3 \cdot 10^{-1}-13$ cts s $^{-1}$ range and therefore should not affect our conclusions in any significant way. The compilation of galactic X-ray binaries with type, optical companion, average flux and, if available, distance and average luminosity is given in the appendix, chapter 8 . The resulting $\log (\mathrm{N})-\log (\mathrm{S})$ relations for LMXBs and HMXBs are shown in Fig. 2.5.
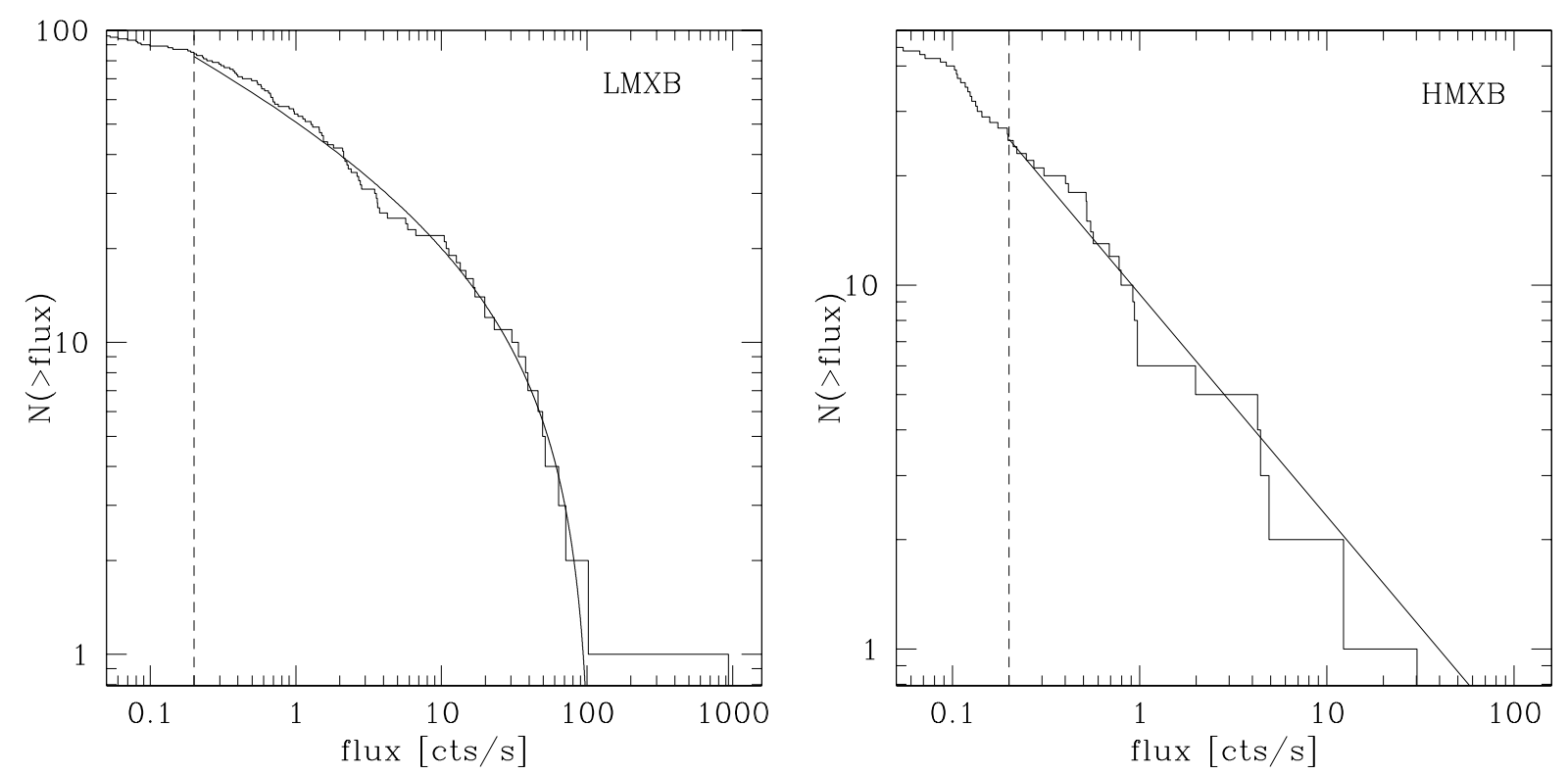

Figure 2.5.: Number-flux relation for galactic X-ray binaries. The vertical dashed line corresponds to our completeness limit of $0.2 \mathrm{cts} \mathrm{s}^{-1}$. The solid lines are the best fit models to the ASM data - a power law for HMXBs and a power law with cutoff in the differential $\log (\mathrm{N})-$ $\log (\mathrm{S})$ distributions at $110 \mathrm{cts} \mathrm{s}^{-1}$ for LMXBs (see Eqs.(2.2) and (2.3)).

To fit the observed $\log (\mathrm{N})-\log (\mathrm{S})$ distributions we used the usual power law in the form:

$$
N(>S)=k \cdot S^{-a}
$$

where $N(>S)$ is the number of sources with fluxes higher than $S, a$ is the slope, and $k$ the normalisation. $S$ is measured in ASM cts s${ }^{-1}$. In order to calculate the best fit values of the parameters we use a Maximum-Likelihood method in the form suggested by Murdoch \& Crawford (1973). This implementation of the M-L method takes into account the errors 
associated with the flux. Since the systematic error dominates the averaged flux error we used the value of $0.05 \mathrm{cts} \mathrm{s}^{-1}$ from chapter 2.1.1 as an estimate of the error. The error is assumed to be Gaussian. Only sources with an averaged flux above $0.2 \mathrm{cts} \mathrm{s}^{-1}$ were used in the fit. The best fit values for different types of Galactic sources are given in Table 2.2. The errors given are an estimate of the $1 \sigma$ errors for one parameter of interest derived from the Maximum-Likelihood method. In order to characterise the quality of the fit we used the Kolmogorov-Smirnov test.

TABLE 2.2.: The best fit values for the number-flux relation for different classes of galactic sources from the ASM catalogue.

\begin{tabular}{|c|r|c|c|c|c|}
\hline Subsample & $\begin{array}{r}\text { Source number } \\
\text { /all sources }\end{array}$ & $\begin{array}{c}\text { cutoff } \\
\left.\text { [cts s }^{-1}\right]\end{array}$ & normalisation & slope & $\begin{array}{c}\text { fit quality } \\
\text { (K-S test) }\end{array}$ \\
\hline all galactic & $131 / 217^{(2)}$ & 110 & 88 & $0.34 \pm 0.05$ & $92 \%$ \\
& $132 / 217$ & - & 72 & $0.41 \pm 0.04$ & $51 \%$ \\
LMXB & $83 / 105^{(2)}$ & 110 & 83 & $0.2 \pm 0.06$ & $71 \%$ \\
& $84 / 105$ & - & 56 & $0.3 \pm 0.05$ & $0.5 \%$ \\
HMXB & $25 / 51$ & - & 9.4 & $0.61_{-0.12}^{+0.14}$ & $46 \%$ \\
SNR & $6 / 7$ & - & 4.8 & $0.36_{-0.19}^{+0.22}$ & $98 \%$ \\
CV & $5 / 10$ & - & 0.5 & $1.68 \pm 0.61$ & $98 \%$ \\
\hline
\end{tabular}

(1) Number of sources above the completeness limit of $0.2 \mathrm{cts} \mathrm{s}^{-1}$.

(2) For fits with a cutoff the brightest source, Sco X-1, was excluded.

As is obvious from Fig. 2.5 and the results of the K-S test (Table 2.2) a simple power law distribution does not describe the observed $\log (\mathrm{N})-\log (\mathrm{S})$ relation for LMXBs. A gradual steepening of the $\log (\mathrm{N})-\log (\mathrm{S})$ relation occurs towards higher fluxes. Similar behaviour was also found by UHURU (Matilsky et al. 1973) and OSO-7 (Markert et al. 1979). We therefore modified the simple power law in the form:

$$
N(>S)=k \cdot\left(S^{-a}-S_{\text {max }}^{-a}\right)
$$

This corresponds to a cutoff in the differential $\log (\mathrm{N})-\log (\mathrm{S})$ relation at flux $S=S_{\text {max }}$. The value of the cutoff was chosen to $S_{\max }=110 \mathrm{cts} \mathrm{s}^{-1}$. The results, however, are not very sensitive to the actual value of $S_{\max }$. The above value of $S_{\max }$ corresponds to the ASM flux from a $1.4 \mathrm{M}_{\odot}$ neutron star located at a distance of $6.5 \mathrm{kpc}$ (average distance of LMXBs from the Sun) and radiating at Eddington luminosity. For fitting the $\log (\mathrm{N})-\log (\mathrm{S})$ of all galactic and LMXB sources with cutoff we excluded the brightest source, Sco X-1, from the sample since its flux is far higher than the cutoff. As can be seen from Table 2.2 and Fig. 2.5 introduction of the cutoff significantly improves the quality of the fit for LMXBs. On the other hand it does not change significantly the results for other types of Galactic sources, especially HMXBs. Note that the steepening of the $\log (\mathrm{N})-\log (\mathrm{S})$ for LMXBs is not an artifact of the incompleteness of the source sample at low fluxes. The numbers do not change qualitatively if we increase the low flux limit by a factor of 2 - the values of K-S probability are $6 \%$ and $68 \%$ for a single power law and a power law with cutoff in the form of Eq. (2.3), respectively. 


\section{Milky Way Luminosity functions}

\subsection{Distribution of $\mathrm{X}$-ray binaries in the Galaxy}

Progress in the number of distance determinations and identifications of secondary stars in X-ray binaries in the last decade opens the opportunity to study the 3-D distribution of XRBs in more detail than was previously possible. Notwithstanding the still relatively small number of X-ray sources and the sometimes poor accuracy of distance determinations it is now possible to compare the observed distribution of XRBs with theoretical expectations. This is not only interesting in itself but, because of the flux limited nature of the ASM sample, knowledge of the spatial distribution is required in order to derive the luminosity function. Due to the above mentioned uncertainties and the flux limitation of the sample it is still not possible to unambiguously determine shape and parameters of the XRB distribution. We therefore adopted an approach in which we use the standard model of the stellar mass distribution in the Galaxy as a starting point and adjust, whenever possible, its parameters to fit observed distributions of low and high mass X-ray binaries. As the luminosity function depends somewhat on the assumed spatial distribution, we verify that variations of the parameters, which can not be determined from the data do not affect derived luminosity functions significantly.

\subsubsection{Angular distribution of $\mathrm{X}$-ray binaries}

The all-sky map shown in Fig. 2.1 demonstrates vividly that the angular distributions of high and low mass X-ray binaries over the sky differ significantly. This fact is further illustrated by the angular distributions against Galactic latitude and longitude shown in Fig. 3.1. The figures illustrate the well-known fact that HMXBs are strongly concentrated towards the Galactic plane. In addition drastic difference in the longitude distributions of HMXBs and LMXBs can be noticed, with the latter significantly concentrated towards the Galactic Centre/Bulge and the former distributed in clumps approximately coinciding with the location of tangential points of the spiral arms,see e.g. Englmaier \& Gerhard (1999); Simonson (1976).

\subsubsection{Source distances and 3-D distribution of $X$-ray binaries}

In order to study the spatial distribution of X-ray binaries we collected source distances from the literature. We found distances for 140 X-ray binaries from the ASM sample. For X-ray binaries with an average flux above the ASM completeness limit, used for constructing the 

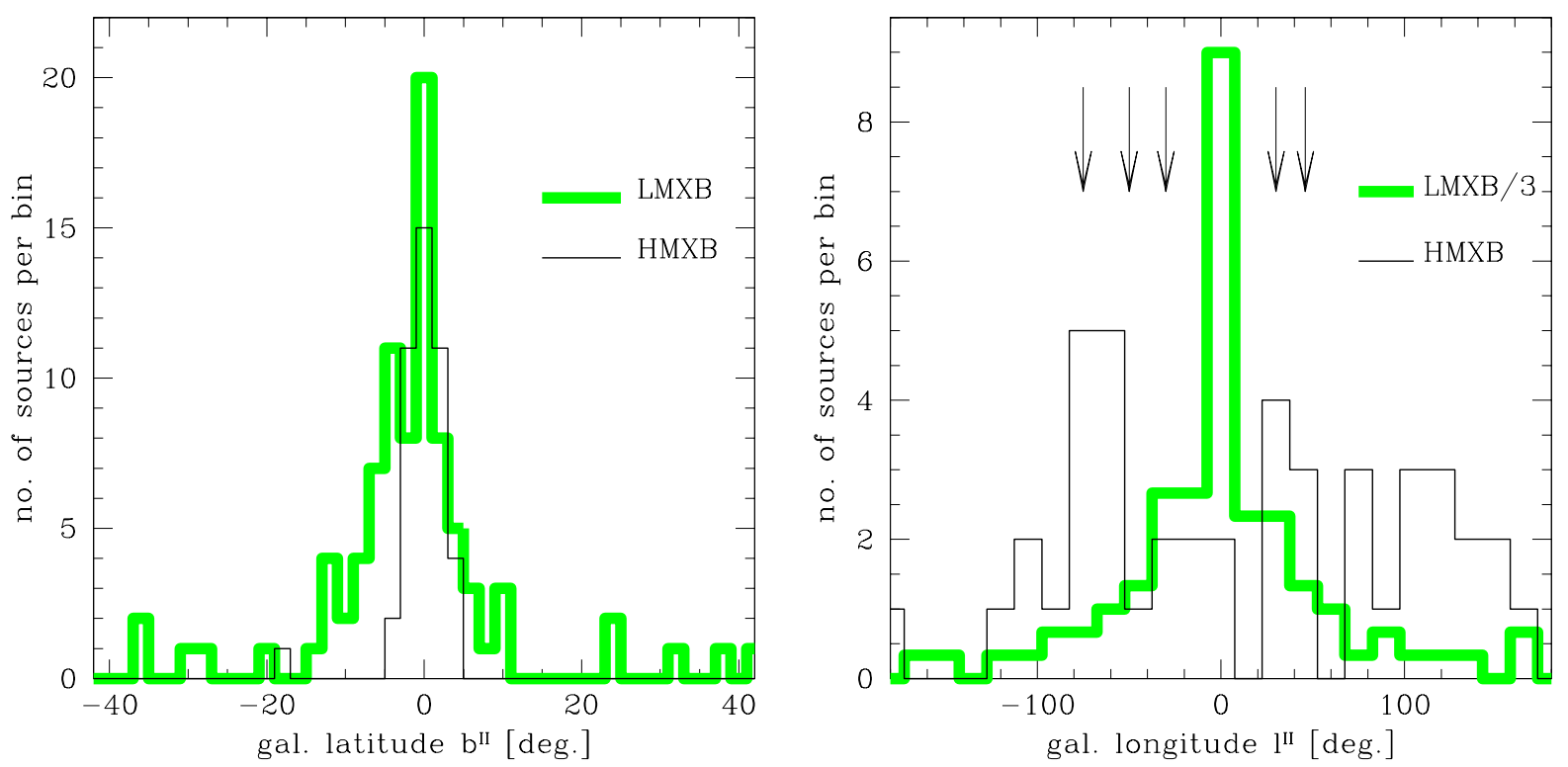

Figure 3.1.: The distribution of Galactic HMXBs (solid lines) and LMXBs (thick grey lines) against Galactic latitude $b^{I I}$ (left panel) and longitude $l^{I I}$ (right panel). The distribution against $b^{I I}$ of HMXBs shows a stronger concentration towards the Galactic plane compared to LMXBs. Along $l^{I I}$ LMXBs show a strong concentration in the direction towards the Galactic centre. The arrows in the right panel mark the positions of the tangential points of spiral arms. The broad hump in the HMXB distribution at $l^{I I}=100^{\circ}-160^{\circ}$ is mostly composed of relatively low luminosity sources in the Perseus and Cygnus arms. Note that on the right panel the number of LMXBs is divided by 3 .

luminosity functions in chapter 5.1.2, distances were determined for all but 8 sources. In cases when the published distance estimates disagree significantly we used the least model dependent estimates or their average. For the compilation of the source distances see the appendix, chapter 8 . The spatial distribution of X-ray binaries in various projections is shown in Fig. 3.2-3.4.

In terms of the spatial distribution of X-ray binaries this thesis elaborates on works done earlier that also distinguished between low and high mass systems but used substantially smaller samples.

Previously White et al. (1980), Lamb et al. (1980), Nagase (1989) and Verbunt (1996) noted the correlation of the positions of accreting X-ray pulsars with high mass companions with the location of spiral arm features of the Milky Way. Based on a larger sample of HMXBs with measured distances we show that indeed the spatial distribution of HMXBs follows the spiral structure of the Galaxy.

Using distance estimates and angular distribution of LMXBs van Paradijs \& White (1995) and White \& van Paradijs (1996) investigated the spatial distribution of LMXBs and BHC in our Galaxy, particularly in the Galactic disk. They estimated values for the vertical (290 pc and $710 \mathrm{pc}$ for BHC and NS binaries) and radial scales (4.5 kpc for NS 


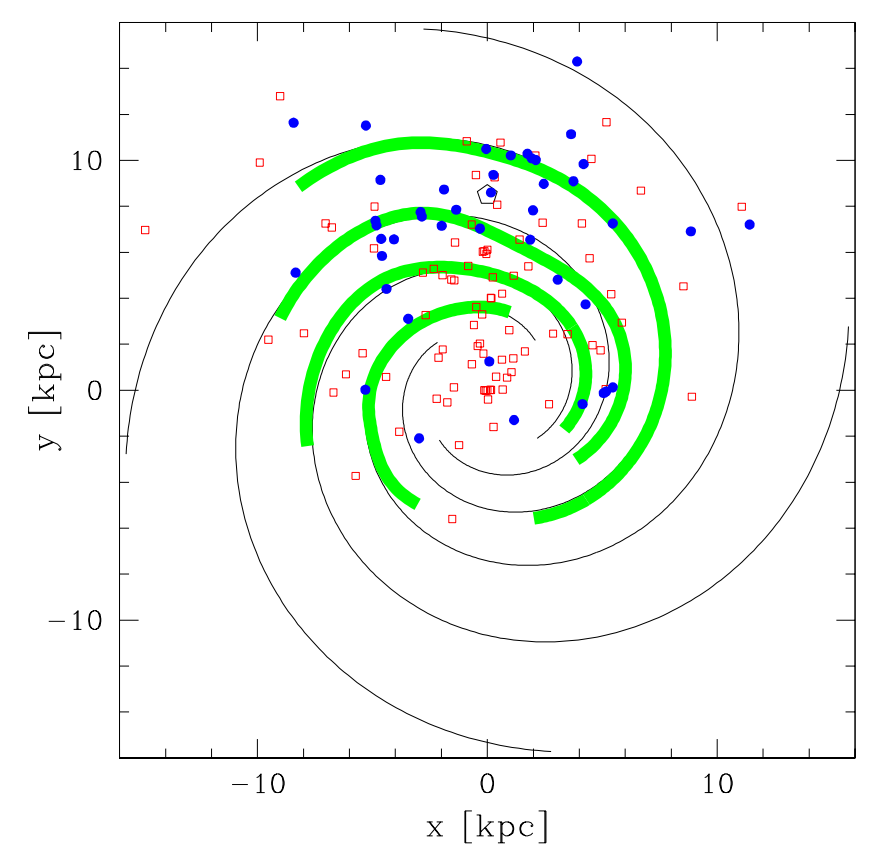

Figure 3.2.: Face-on view of the Galaxy - distribution of low mass (open squares) and high mass (filled circles) X-ray binaries. The origin of the coordinate is at the Galactic Centre. The Sun is located at $\mathrm{x}=0, \mathrm{y}=8.5$ (marked by the pentagon). The thin solid line shows logarithmic 4armed $(m=4)$ spiral model with pitch angle of $12^{\circ}$ (e.g. Vallée (1995)). The thick solid lines show the spiral model of the Galaxy based on optical and radio observation of the giant HII regions (Georgelin \& Georgelin (1976), Taylor \& Cordes (1993)). The fact that the majority of sources is located at $y>0$ is due to the flux limited nature of the ASM sample and incompleteness of the optical identifications/distance measurements at the large distances from the Sun (see discussion in the text).

binaries) of the disk. These values are in general agreement with those obtained in this thesis, that are based on a considerably larger number of sources. Grebenev et al. (1996) found good agreement between the source distribution observed by ART-P/GRANAT in the Galactic Centre region and the stellar mass distribution in the Galactic Bulge. We thus have a reasonably good knowledge about the distribution of LMXBs in the Galaxy.

\subsubsection{The Galaxy model}

As a starting point in constructing the spatial distribution of X-ray binaries we employ the standard three component model of the stellar mass distribution in the Galaxy (Bahcall \& Soneira 1980), consisting of bulge, disk and spheroid. The parameterisation of bulge and disk is taken from Dehnen \& Binney (1998) and for the spheroid we take the model of Bahcall \& Soneira (1980):

$$
\begin{gathered}
\rho_{\text {Bulge }}=\rho_{0, \text { Bulge }} \cdot\left(\frac{\sqrt{r^{2}+\frac{z^{2}}{q^{2}}}}{r_{0}}\right)^{-\gamma} \cdot \exp \left(-\frac{r^{2}+\frac{z^{2}}{q^{2}}}{r_{t}^{2}}\right) \\
\rho_{\text {Disk }}=\rho_{0, \text { Disk }} \cdot \exp \left(-\frac{r_{m}}{r}-\frac{r}{r_{d}}-\frac{|z|}{r_{z}}\right) \\
\rho_{\text {Sphere }}=\rho_{0, \text { Sphere }} \cdot \frac{\exp \left(-b \cdot\left(\frac{R}{R_{e}}\right)^{1 / 4}\right)}{\left(\frac{R}{R_{e}}\right)^{7 / 8}},
\end{gathered}
$$




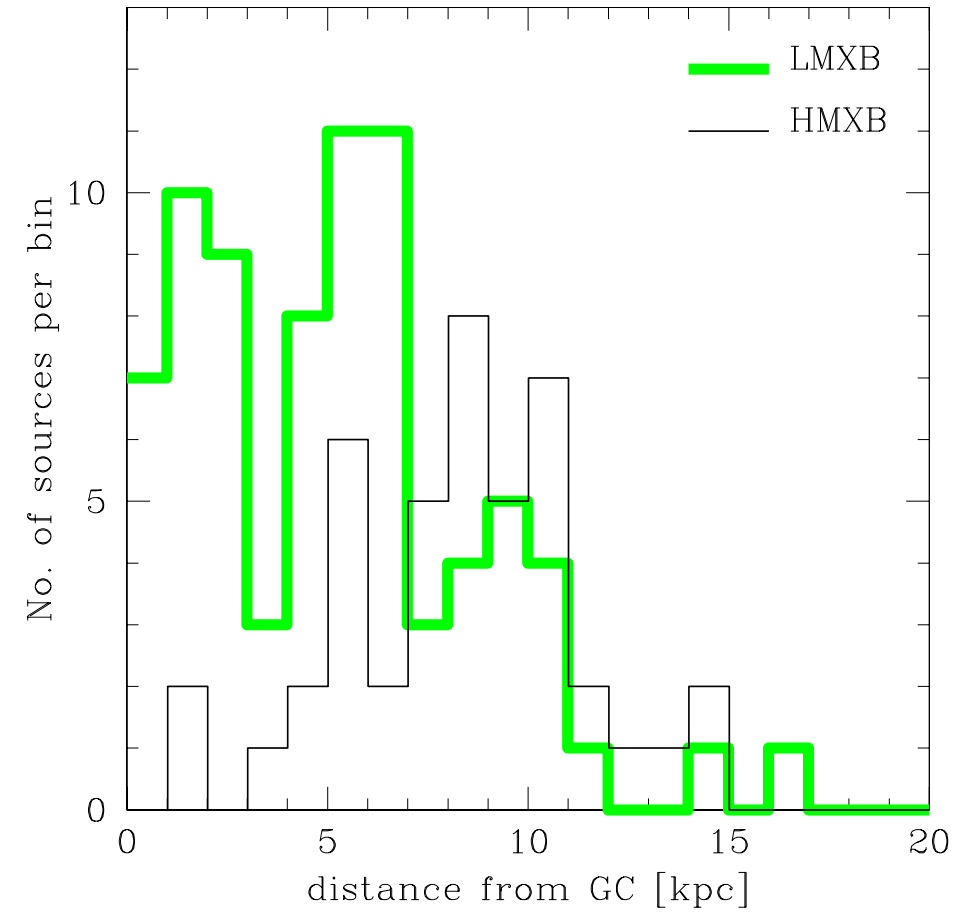

FiguRE 3.3.: Radial distributions of high mass (solid histogram) and low mass (thick grey histogram) Xray binaries. The projected distance is defined as $\sqrt{x^{2}+y^{2}}$, where $x$ and $y$ are Cartesian coordinates in the Galactic plane, see Fig. 3.2. Note that the plotted distributions are not corrected for the volume of cylindrical shells $(\propto r)$.

where $\rho_{0, \text { Bulge }}, \rho_{0, \text { Disk }}$ and $\rho_{0, \text { Sphere }}$ are the normalisations, $r$ is the distance in the plane from the galactic centre, $z$ is the distance perpendicular to the galactic plane, and $R$ is the distance from the galactic centre in spherical coordinates. All distances are in kiloparsec. Meaning and values for other parameters are given in Table 3.1.

In the standard Galaxy model the mass ratios of the components are about 2:1:0.3 for disk:bulge:spheroid. These numbers follow from the model using normalisations for the disk, $\rho_{0, \text { Disk }}=0.05 \mathrm{M}_{\odot} \mathrm{pc}^{-3}$, and spheroid population, $\rho_{0, \text { Sphere }}=1 / 500 \cdot \rho_{0, \text { Disk }}$, observed in the vicinity of the Sun (Zombeck 1990) and a bulge mass of about $\sim 1.3 \cdot 10^{10} \mathrm{M}_{\odot}$ (Dwek et al. 1995). All these masses refer to baryonic mass in the stars.

All three components of the standard Galaxy model were used to construct the spatial distribution of LMXB. The spheroid component with appropriately adjusted normalisation was used to account for the population of globular cluster sources. Based on the observed distribution and theoretical expectation that HMXBs trace the star forming regions in the Galaxy, only the disk component was used for the spatial distribution of HMXBs.

Several parameters, namely vertical scale height of the disk and relative normalisation of the spheroid for the LMXBs, can be determined directly from our sample of X-ray binaries. For these parameters we used the best fit values inferred by the data. For the rest of the parameters we accepted standard values for the stellar mass distribution in the Galaxy. The final set of the parameters is summarised in Table 3.1.

The disk component of the standard Galaxy model was modified in order to account for the Galactic spiral structure. The description of the spiral arms is based on the model of 

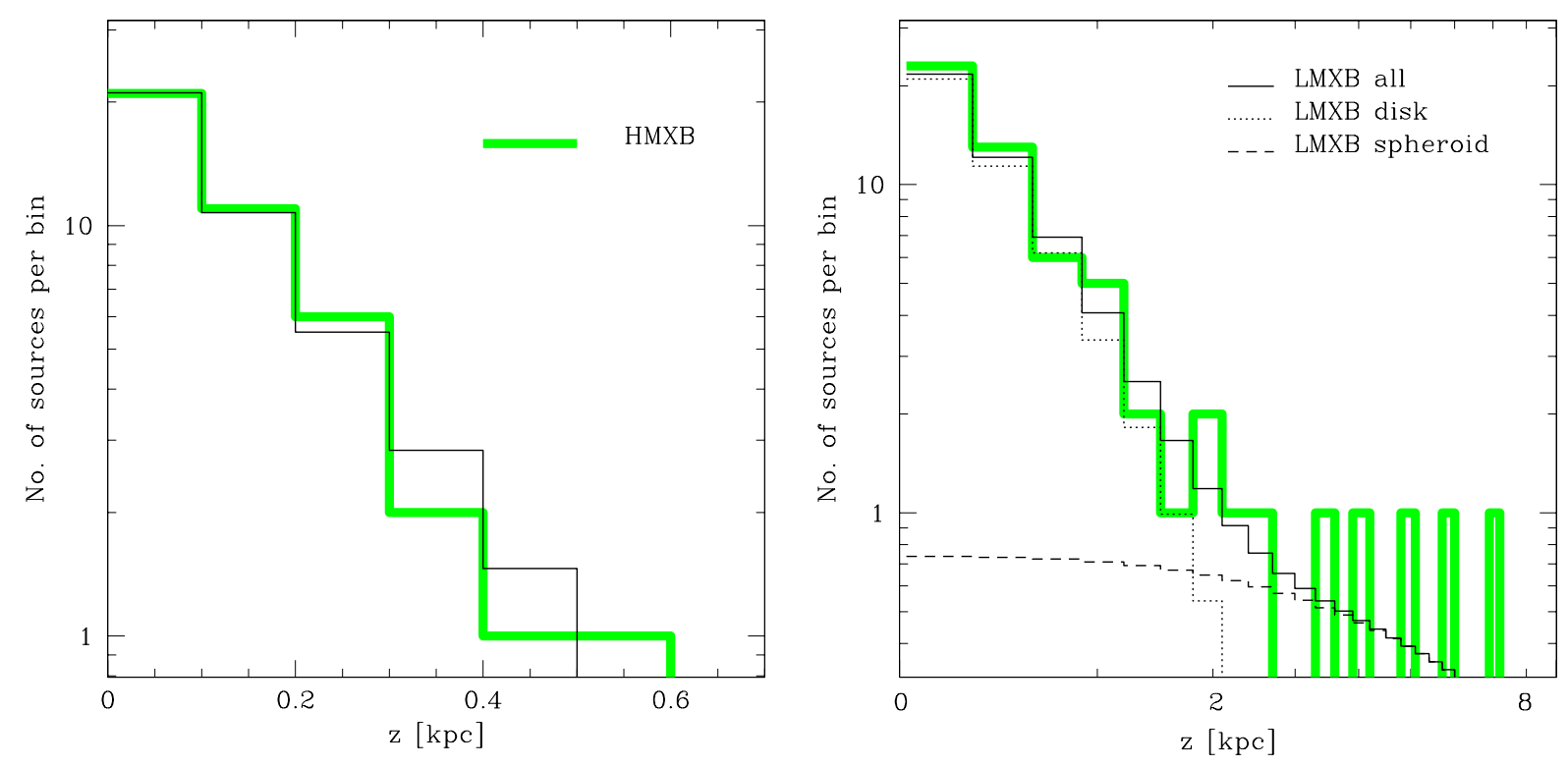

Figure 3.4.: Vertical distributions of high mass (left panel) and low mass (right panel) X-ray binaries. The vertical distributions were summed over northern and southern galactic hemispheres. In the case of LMXBs only sources with $R>3.5 \mathrm{kpc}$ were used, to exclude bulge sources. The thick grey solid lines show the observed distributions and the thin solid and dashed lines the expected distributions for an exponential disk with $150 \mathrm{pc}$ scale height for HMXBs, and an exponential with scale height $410 \mathrm{pc}$ and a $25 \%$ contribution of the spheroid for LMXBs, respectively. For the assumed model see Eqs. $(3.2,3.3)$.

Georgelin \& Georgelin (1976) derived from the distribution of HII regions. To include it into our Galaxy model we used the FORTRAN code provided by Taylor \& Cordes (1993). The spiral arms computed in this way are shown in Fig. 3.2 by thick grey lines. This empirical model is close but not identical to a 4 arm logarithmic spiral with pitch angle of $12^{\circ}$ (e.g. Vallée (1995)) shown in Fig. 3.2 by thin solid lines.

In the following two sub-chapters we discuss spatial distribution of HMXBs and LMXBs in more detail.

\subsubsection{High mass $X$-ray binaries}

The angular distribution of HMXBs in Fig. 3.1 shows signatures of the Galactic spiral structure. These signatures are clearly seen in the distribution of sources over galactic longitude which shows maxima approximately consistent with directions towards tangential points of the spiral arms. No significant peak in the direction to the Galactic centre is present. The signatures of the spiral structure become more evident in the 3-D distribution of the smaller sample of sources for which distance measurements are available, Figs. 3.2,3.3. The radial distribution (Fig. 3.3) shows pronounced peaks at the locations of the major spiral arms and is similar to that of primary tracers of the Galactic spiral structure 
TABLE 3.1.: The parameters of the standard Galaxy model.

\begin{tabular}{|c|c|c|c|}
\hline parameter & meaning & \multicolumn{2}{|c|}{ value } \\
\cline { 3 - 4 } & & HMXB & LMXB \\
\hline$q$ & oblateness of bulge & - & 0.6 \\
$\gamma$ & - & - & 1.8 \\
$R_{e}$ & scale length of spheroid & - & $2.8 \mathrm{kpc}$ \\
$b$ & - & - & 7.669 \\
$r_{0}$ & scale length of bulge & - & $1.9 \mathrm{kpc}$ \\
$r_{t}$ & truncation radius of bulge & $3.5 \mathrm{kpc}$ & $3.5 \mathrm{kpc}$ \\
$r_{d}$ & scale length of disk & $150 \mathrm{pc}$ & $410 \mathrm{pc}$ \\
$r_{z}$ & vertical scale of disk & $6.5 \mathrm{kpc}$ & $6.5 \mathrm{kpc}$ \\
$r_{m}$ & inner disk cut-off & $1: 0: 0$ & $2: 1: 0.8$ \\
$R_{\text {mass }}$ & mass ratios Disk:Bulge:Spheroid & &
\end{tabular}

- giant HII regions (e.g. Downes et al. (1980)) and warm molecular clouds (e.g. Solomon et al. (1985)). In particular, the central $\sim 3-4 \mathrm{kpc}$ region of the Galaxy is almost void of HMXB well in accordance with the radial distribution of the giant HII regions and warm $\mathrm{CO}$ clouds. This appears to correspond to the interior of the 4-kpc molecular ring.

The vertical distribution of HMXBs is significantly more concentrated towards the Galactic Plane and sufficiently well described by a simple exponential with a scale height of 150 pc as shown in the left panel of Fig. 3.4.

Based on theoretical expectations and on the data shown in Fig. 2.1,3.1,3.3,3.4 we included only the disk component in the volume density distribution HMXBs. It is clear however that a simple exponential disk is not a good description for the radial distribution of HMXB. Therefore, following Dehnen \& Binney (1998) we assumed the disk density distribution in the form given by Eq. (3.2), where the first term in the exponential allows for the central density depression. To describe the observed central depression for HMXBs a rather large value of $r_{m} \approx 6-7 \mathrm{kpc}$ is required (cf. $r_{m}=4 \mathrm{kpc}$ from Dehnen \& Binney (1998)). The spiral arms were assumed to have a Gaussian density profile along the Galactic Plane:

$$
\rho_{\text {Spiral }} \propto \sum_{j=1}^{j=4} \exp \left(-\left(\frac{s_{j}}{w_{a}}\right)^{2}\right),
$$

where $w_{a}=600 \mathrm{pc}$ is the width of the spiral arm, and $s_{j}$ is the distance to the nearest point of the spiral arm $j$ projected to the Galactic Plane:

$$
s_{j}=\sqrt{\left(x-x_{j}^{\prime}\right)^{2}+\left(y-y_{j}^{\prime}\right)^{2}} .
$$

In order to account for the spiral structure the disk density, Eq. (3.2), was multiplied by $\rho_{\text {Spiral }}$ :

$$
\rho_{\text {Disk }}^{H M X B} \propto \rho_{\text {Disk }} \cdot \rho_{\text {Spiral }}
$$




\subsubsection{Low mass $X$-ray binaries}

Contrary to HMXB, the angular distribution of LMXBs is strongly peaked in direction to the Galactic centre and declines gradually along the Galactic plane, see Fig. 3.1. The central $\sim 2$ kpc region is densely populated with Galactic Bulge LMXB sources and contains $\sim 1 / 3$ of the LMXBs from our flux limited sample (Fig. 3.3). A noticeable feature of the radial distribution of LMXB is the pronounced minimum at $\sim 3-4 \mathrm{kpc}$. This minimum approximately coincides with the $\sim 1-3 \mathrm{kpc}$ gap in the distribution of the molecular gas and the $\sim 2.2 \mathrm{kpc}$ minimum in the density of infrared light distribution in the Galaxy (Binney et al. 1997) and probably separates bulge sources from the disk population. Similar to HMXBs, the signatures of the spiral structure might be present in the radial distribution although they are less pronounced.

The vertical distribution outside the bulge (Fig. 3.4) is significantly broader than that of HMXBs and includes a number of sources at high galactic $z$. A formal fit to the observed distribution with an exponential law results in a large scale height of $950 \pm 130 \mathrm{pc}$, which is close to the value of 710 pc obtained by van Paradijs \& White (1995) for NS LMXBs. However, due to presence of a tail of sources at $|z|>1.5-2 \mathrm{kpc}$, the observed z-distributions cannot be adequately described by a simple exponential law. As only three out of nine sources at $|z|>2 \mathrm{kpc}$ are located in globular clusters, this tail of high-z sources cannot be solely due to the globular cluster component. A possible mechanism - a kick received by a compact object during the SN explosion, was considered e.g. by van Paradijs \& White (1995). The relatively small number of high-z sources does not allow one to determine the shape of their distribution based on the data only. In order to account for the high-z sources and the LMXB sources in globular clusters we chose to include in the spatial distribution of LMXBs the spheroid component described by a de Vaucouleurs profile (Eq. (3.3)). Note that a de Vaucouleurs profile correctly represents the distribution of globular clusters. The overall vertical distribution can be adequately represented by a sum of an exponential law with a scale height of $410_{-80}^{+100} \mathrm{pc}$ and a de Vaucouleurs profile with the parameters given in Table 3.1. The spheroid component represented by the de Vaucouleurs profile contains $\mathrm{a} \sim 25 \%$ of the total number of LMXBs. Note, that this number is by a factor of $\sim 2-3$ larger than the mass fraction of the stellar spheroid in the standard Galaxy model. The enhanced fraction of the spheroid component is generally consistent with the fact, that the number of X-ray sources per unit mass is $\sim 100$ times higher in the globular clusters than in the Galactic disk and 12 out of 104 LMXBs in our sample are globular cluster sources.

The angular resolution of the ASM instrument does not permit to study in detail the very central region of the Galaxy which is characterised by the highest volume and surface density of X-ray binaries. Based on GRANAT/ART-P data having significantly better sensitivity and angular resolution, Grebenev et al. (1996) showed that the distribution of the surface density of X-ray binaries in the central $8^{\circ} \times 8^{\circ}$ of the Galaxy is consistent with the stellar mass distribution in the Galactic Bulge.

To conclude, our model of the volume density distribution of LMXBs includes all three components of the standard model of the Galaxy: bulge, disk and spheroid with the diskto-spheroid mass ratio decreased to $4: 1$. Similarly to HMXBs, $r_{m} \approx 6-7 \mathrm{kpc}$ is required 
to describe the central density suppression of the disk population. The modulation of the disk component by the spiral pattern at the $20 \%$ level was also included:

$$
\rho_{\text {Disk }}^{\mathrm{LMXB}} \propto \rho_{\text {Disk }} \cdot\left(1+0.2 \cdot \rho_{\text {Spiral }}\right)
$$

where $\rho_{\text {Disk }}$ is given by Eq. (3.2) and $\rho_{\text {Spiral }}$ - by Eq. (3.4).

\subsubsection{Completeness of the sample of the distance measurements.}

The fact that the majority of the sources in Fig. 3.2 is located at $y>0$ is related to the flux limited nature of the ASM sample (obviously it is easier to observe weak sources located closer to the Sun) and to the incompleteness of the available distance measurements (more difficult to measure the distance to a more distant source). The 3-D distribution of X-ray binaries enables one to check the latter effect.

Plotted in Fig. 3.5 is the distribution of LMXB sources with luminosities $L_{X}>4 \cdot 10^{36}$ erg s${ }^{-1}$ over the distance from the Sun. For the ASM completeness flux limit of 0.2 cts $\mathrm{s}^{-1}$, sources with $L_{X}>4 \cdot 10^{36} \mathrm{erg} \mathrm{s}^{-1}$ should be visible up to a distance of $\approx 20 \mathrm{kpc}$. However, comparison with the expected distribution computed using the LMXB volume density distribution constructed in chapter 3.1.5 shows an increasing deficiency of sources at distances $\gtrsim 10-15 \mathrm{kpc}$. In total $\sim 14$ sources in the distance range of $10-20 \mathrm{kpc}$ are "missing". These "missing" sources should be hidden among the $\sim 20$ unclassified sources in the ASM catalogue for which no optical identification/distance determinations are available.

Recent observations by Kuijken \& Rich (2001) lend support to this interpretation. They measured proper motions of blue and red giants in direction to the Galactic centre. The red giants, concentrated in the Galactic bulge, have a velocity dispersion in Galactic coordinates, $b^{I I}$ versus $l^{I I}$, symmetric around zero. However, blue giants, located in the disk, have a velocity dispersion asymmetric around zero with respect to $l^{I I}$ which means that there is a net motion of the observed blue giants in one direction. Interpreting this as the motion of the disk around the Galactic centre, it also means that there is a deficit of the observed blue giants on the far side of the Galaxy (cf. Fig. 3.5).

This comparison (Fig. 3.5) shows that our sample of optical identifications/distance measurements for LMXB sources is complete up to a distance of $\sim 10 \mathrm{kpc}$. The significantly smaller number of HMXBs above the ASM completeness flux limit did not permit us to perform a similar analysis for HMXB sources. However, one might expect that due to the higher luminosity of the optical companion the limiting distance for HMXBs is not smaller than for LMXBs. We therefore accepted a value of $D_{\max }=10 \mathrm{kpc}$ as a maximum source distance for the luminosity function calculation for both types of sources described in the next chapter. 


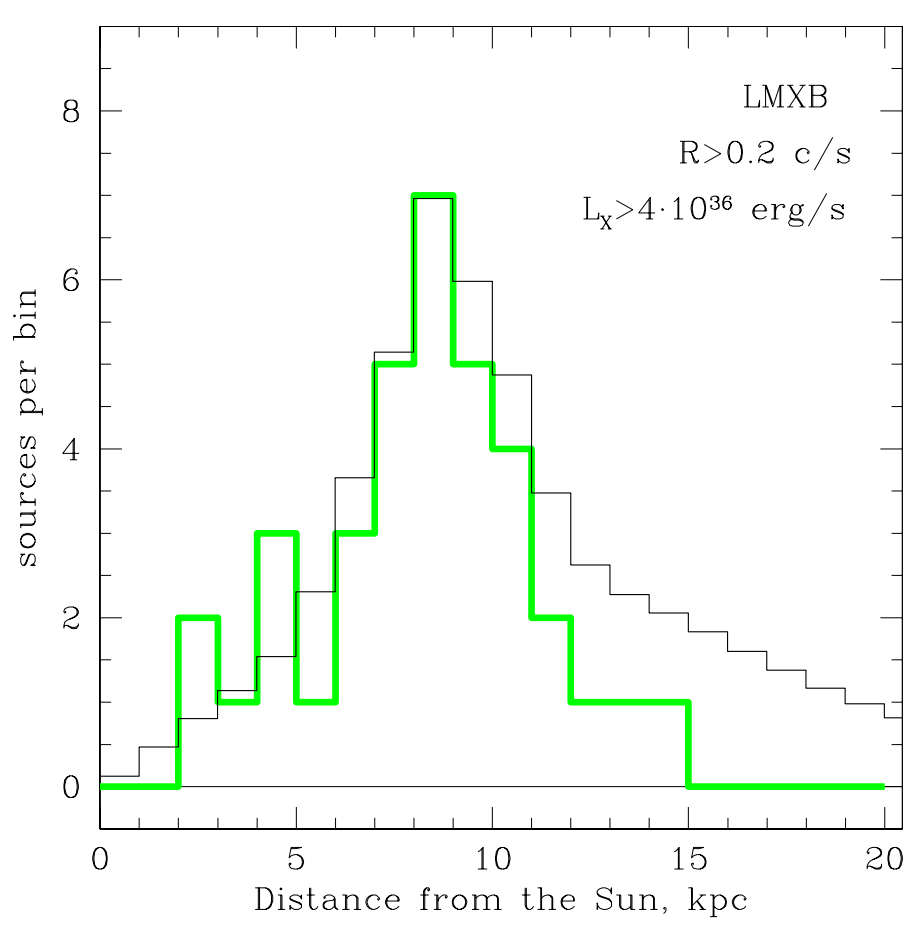

FIgURE 3.5.: Distribution of the LMXB sources over distance from the Sun (thick grey histogram). Only sources with luminosity $L_{X}>4 \cdot 10^{36}$ $\operatorname{erg~s}^{-1}$ are plotted. Given the ASM completeness flux limit of $0.2 \mathrm{cts} \mathrm{s}^{-1}$, sources with $L_{X}>4 \cdot 10^{36} \mathrm{erg} \mathrm{s}^{-1}$ should be visible from the distance of upto $\approx 20 \mathrm{kpc}$. The thin solid histograms shows the expected distribution of the sources in the model constructed in chapter 3.1.5. Deviation of the observed distribution from the prediction becomes visible at the distance $>10-15 \mathrm{kpc}$.

\subsection{Luminosity function}

Due to the flux limited nature of the ASM sample and incompleteness of the optical identifications/distance measurements beyond $\sim 10 \mathrm{kpc}$, the apparent luminosity function which can be derived straightforwardly from the ASM flux measurements and the source distances (thin line histograms in Fig. 3.7 and 3.8) needs to be corrected for the fraction of the Galaxy observable by ASM. This correction can be performed using the model of the spatial distribution of X-ray binaries constructed in the previous sub-chapters:

$$
\frac{d N}{d L}=\left(\frac{d N}{d L}\right)_{\mathrm{obs}} \times \frac{M(<D(L))}{M_{\text {tot }}}
$$

where $\frac{d N}{d L}$ is the true luminosity function, $\left(\frac{d N}{d L}\right)_{\text {obs }}$ - apparent luminosity function constructed using ASM flux measurements and the source distances, $M(<D)$ - mass of the Galaxy inside distance $D$ from the Sun computed using the volume density distributions for HMXB and LMXB sources from the chapter 3.1, $M_{\text {tot }}$ - total mass of the Galaxy, $D(L)$ is defined by:

$$
D(L)=\min \left(\frac{L}{\sqrt{4 \pi F_{\text {lim }}}}, D_{\max }\right)
$$

where $F_{\text {lim }}$ is the limiting (minimum) flux and $D_{\max }$ - the maximum distance from the Sun of the sources used for constructing the luminosity function. As discussed in the previous chapters we accepted the following selection criteria: $F_{\text {lim }}=0.2 \operatorname{cts~s}^{-1} \approx 6.4 \cdot 10^{-11} \mathrm{erg}$ 


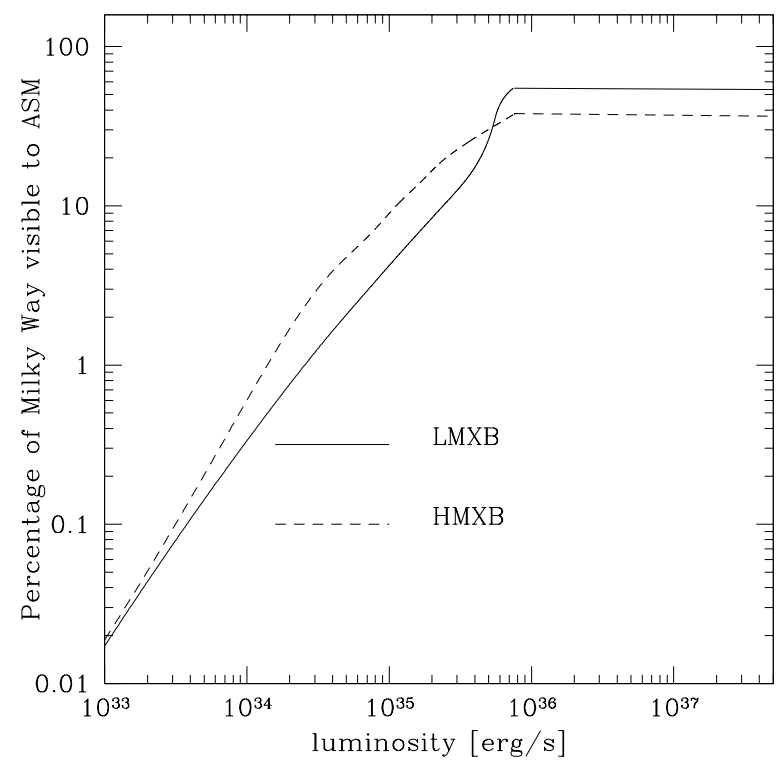

FiguRE 3.6.: Fraction of the mass of the Galaxy visible to ASM with account for the selection criteria described in the text as a function of source luminosity.

$\mathrm{s}^{-1} \mathrm{~cm}^{-2}$, i.e. equal to the completeness flux limit of the ASM catalogue, and $D_{\max }=10$ $\mathrm{kpc}$ - a completeness limit of distance measurements estimated in chapter 3.1.6.

Obviously, for a given flux limit $F_{\text {lim }}$ the mass fraction of the Galaxy $\frac{M(<D(L))}{M_{\text {tot }}}$ is a decreasing function of the source luminosity as shown in Fig. 3.6. For the ASM sensitivity/completeness limit of $\approx 6.4 \cdot 10^{-11} \mathrm{erg} \mathrm{s}^{-1} \mathrm{~cm}^{-2}$ the entire volume inside $D_{\max }=10 \mathrm{kpc}$ from the Sun is observable down to a luminosity of $\approx 10^{36} \mathrm{erg} \mathrm{s}^{-1}$ (the flat part of the curves in Fig. 3.6) below which the mass fraction of the observable part of the Galaxy begins to decrease. As the spatial distributions of HMXB and LMXB sources differ significantly, the volume correction and the luminosity function were calculated separately for HMXBs and LMXBs. The apparent and volume corrected (true) cumulative luminosity functions are presented in Fig. 3.7. Fig. 3.8 shows the corresponding differential distributions binned logarithmically over luminosity.

The cumulative luminosity function of HMXBs (Fig. 3.7, right panel) does not seem to contradict to a power law distribution down to a luminosity of $\sim 2 \cdot 10^{35} \mathrm{erg} \mathrm{s}^{-1}$ with some indication of flattening at lower luminosity. However, limited sensitivity of ASM and correspondingly large values of the correction factor (Fig. 3.6) at low luminosities do not allow one to draw a definite conclusion regarding the shape of the luminosity function at these low luminosities (see comparison with ASCA source counts in chapter 4.1). We therefore fitted the luminosity function of HMXBs in the $L>2 \cdot 10^{35} \mathrm{erg} \mathrm{s}^{-1}$ range with a power law distribution. Using a Maximum-Likelihood method the best fit parameters are:

$$
N(>L)=20 \cdot\left(\frac{L}{10^{36} \mathrm{erg} \mathrm{s}^{-1}}\right)^{-0.64 \pm 0.15}
$$

where $L$ is the source luminosity in $\operatorname{erg~s}^{-1}$ and $N(>L)$ - total number of sources on the sky with luminosity greater than $L$. 

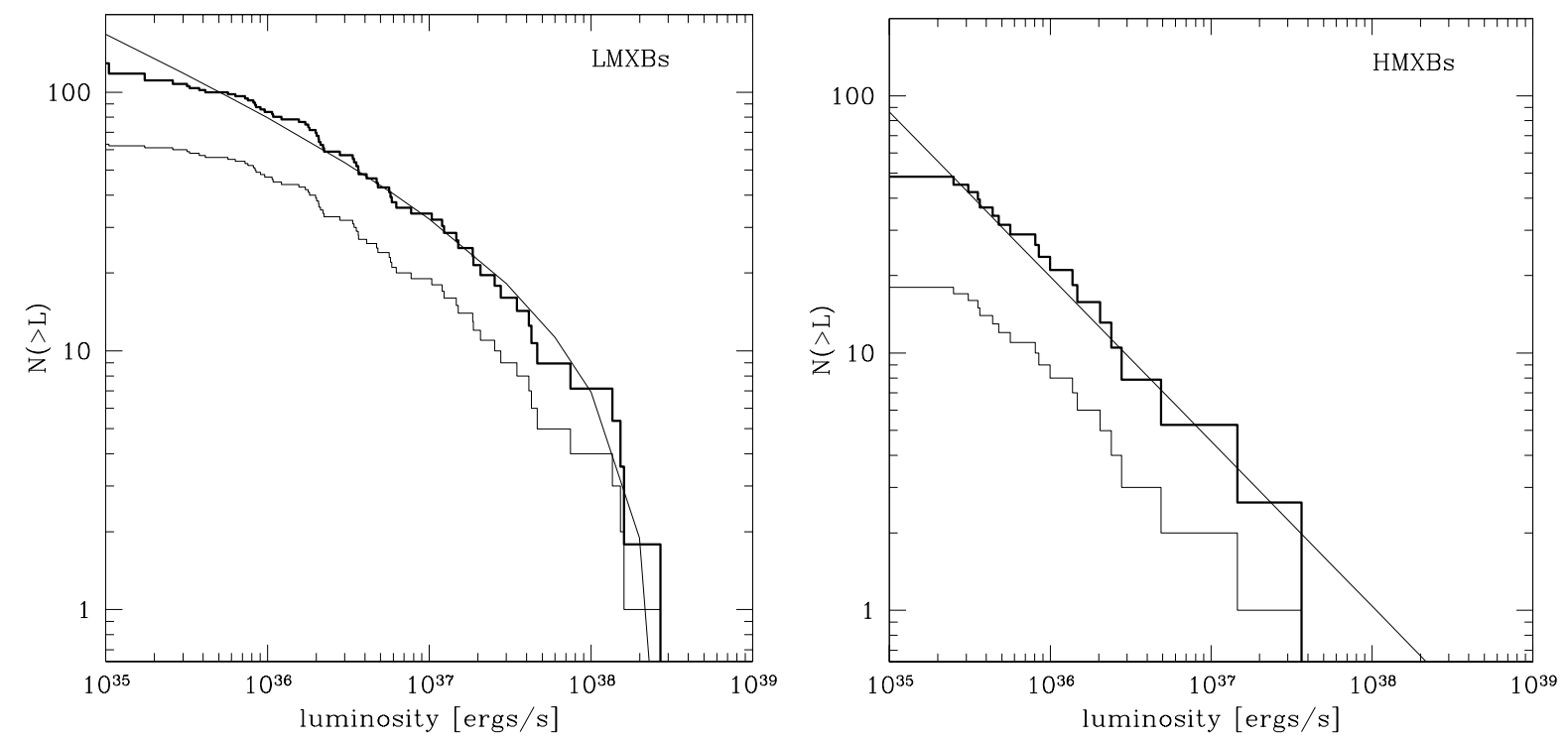

FiguRE 3.7.: The apparent (thin histogram) and volume corrected (thick histogram) cumulative luminosity function for LMXBs and HMXBs. The solid lines are the best fits to the data.
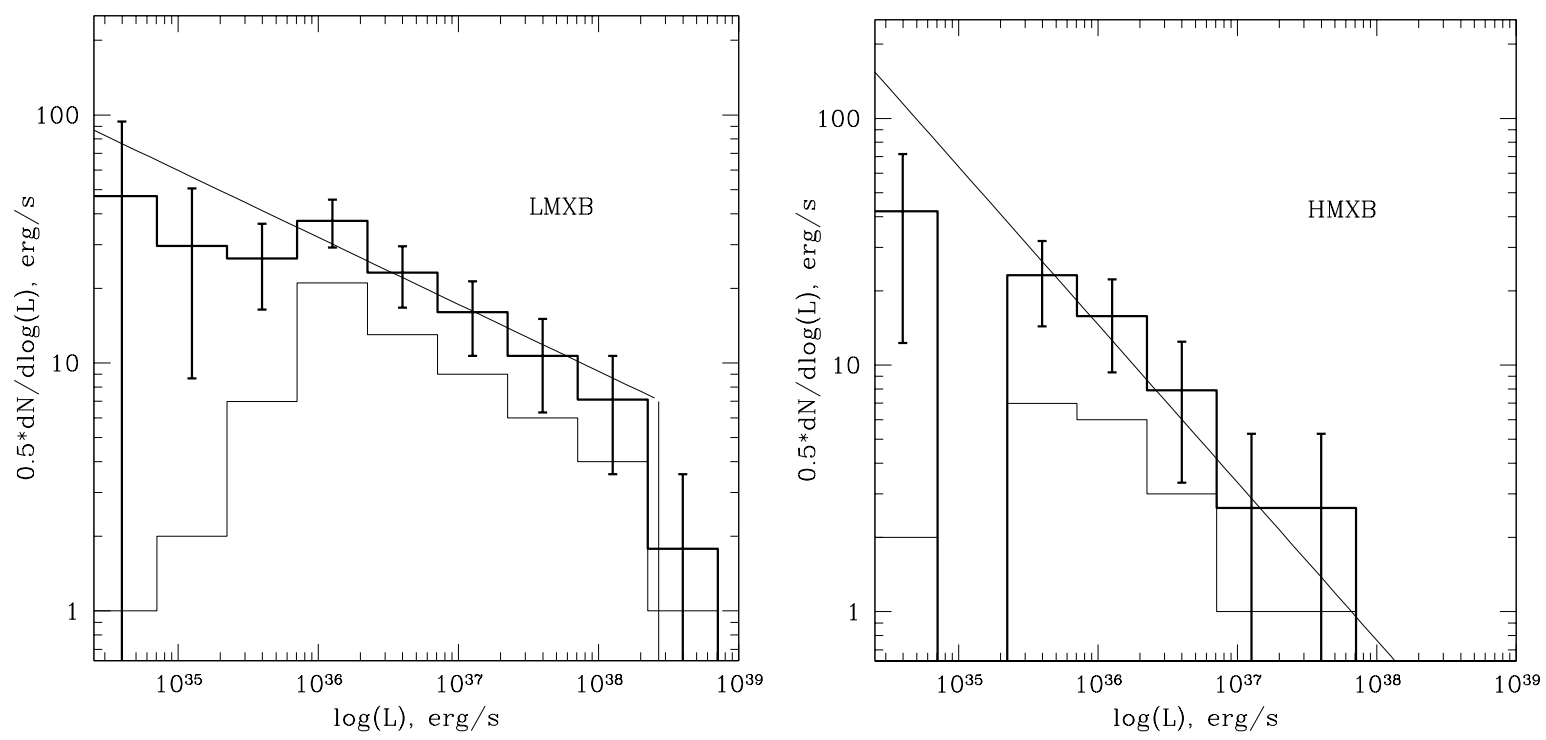

FiguRE 3.8.: The apparent (thin histogram) and volume corrected (thick histogram) differential luminosity function for LMXBs and HMXBs binned into bins with logarithmic with of 0.5. The solid lines are the best fits to the cumulative distributions. The fall-over of the apparent distributions below $\sim 10^{36} \mathrm{erg} \mathrm{s}^{-1}$ are due to the flux limited nature of the ASM sample (see Fig. $3.6)$ 
The shape of both cumulative and differential luminosity function for LMXBs (Figs. 3.7, 3.8, left panels) indicates the presence of a high luminosity cut-off. We fitted the unbinned cumulative distribution with the functional form

$$
N(>L)=A \cdot\left(L^{-\alpha}-L_{\max }^{-\alpha}\right) .
$$

corresponding to a power law differential luminosity function with a sharp cut-off at $L_{\max }$. The value of the cutoff was set equal to to $2.7 \cdot 10^{38} \mathrm{erg} \mathrm{s}^{-1}$ which corresponds to the luminosity of the most luminous source within $10 \mathrm{kpc}$, Sco X-1. The best fit values of other parameters are:

$$
N(>L)=105 \cdot\left(\left(\frac{L}{10^{36} \mathrm{erg} \mathrm{s}^{-1}}\right)^{-0.26 \pm 0.08}-270^{-0.26}\right) .
$$

Note that the smaller number of sources and the steeper slope of luminosity function make the HMXB data insensitive to a high luminosity cut-off above $\sim$ few $\times 10^{36} \mathrm{erg} \mathrm{s}^{-1}$.

\subsubsection{Effect of the Galaxy model on the luminosity function}

From Eq. (3.8) it is clear that the luminosity function depends on the spatial distribution of XRBs in the Galaxy. As discussed above, using the distance measurements available, we were able to determine some of the parameters of their distribution. But the data are not sufficient to determine the entire distribution unambiguously. Thus we had to assume a spatial distribution of XRBs in the Galaxy. In order to investigate the effect of the adopted spatial distribution of X-ray sources on the derived luminosity function we varied our model and computed the respective luminosity functions.

For our analysis we used three different distributions for LMXBs and HMXBs. In the case of HMXBs, only the disk component was included in each of the three distributions. The modulation of the disk distribution by the spiral pattern, when present, was $100 \%$ for HMXB and $20 \%$ for LMXB. The models are:

- Model A: Our primary model constructed in chapter 3.1 and used to derive the luminosity function above (shown as a solid histogram in Fig. 3.9).

- Model B: The same as the model A, except that the inner cut-off of the disk was set to $r_{m}=4 \mathrm{kpc}$ in accordance with the result of Dehnen \& Binney (1998)(dotted histogram in Fig. 3.9).

- Model C: The spheroid component is the same as in Model A. The disk radial distribution is without the inner cut-off, i.e. $r_{m}=0$ and without modulation by the spiral structure. No bulge component is included for either LMXBs or HMXBs. The resulting density distribution is similar to that derived by van Paradijs \& White (1995) for NS LMXBs (dashed histogram in Fig. 3.9). 

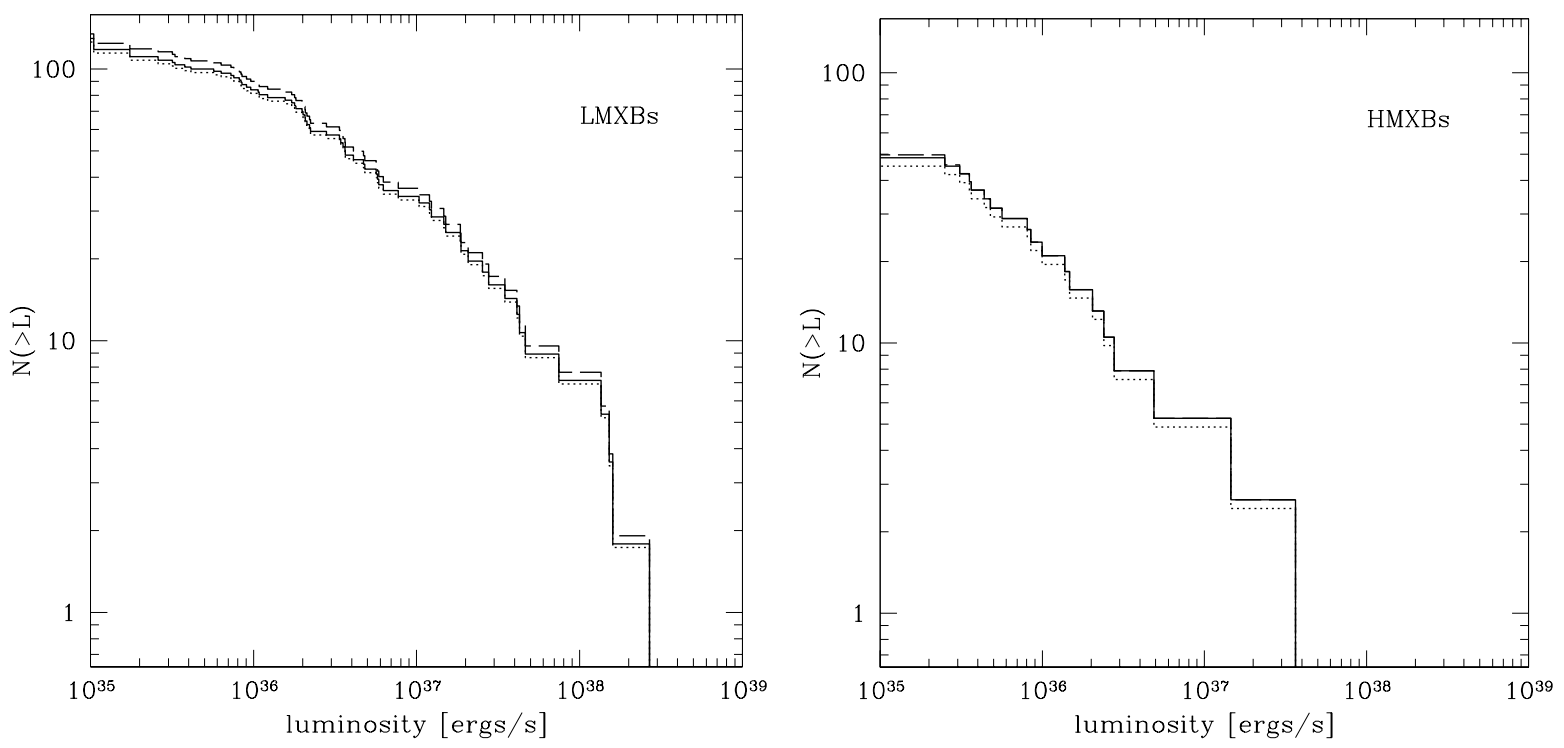

Figure 3.9.: Dependence of the luminosity function on the adopted model of the spatial distribution of XRBs. The figures show the luminosity functions of LMXBs (left panel) and HMXBs (right panel) for three different Galaxy models. The solid, dotted and dashed lines in both panels correspond to the models $\mathrm{A}, \mathrm{B}$ and $\mathrm{C}$.

The resulting luminosity function for each of the three models are shown in Fig. 3.9. It is obvious that there is no strong dependence of the luminosity function on the mass distribution. The slopes vary in the range from $1.28-1.30$ for LMXBs and $1.64-1.72$ for HMXBs. The total number of sources varies from 88 to 90 for LMXBs and from 21 to 26 for HMXBs. It is worth noting that the spiral pattern is no significant factor in the determination of the luminosity function of HMXBs although the spatial distribution shows clear signs of them.

\subsubsection{Total X-ray luminosity of Galactic $\mathrm{X}$-ray binaries}

The total luminosity of all X-ray binaries in the Galaxy is calculated in the following way. Down to a luminosity of $10^{36} \mathrm{erg} \mathrm{s}^{-1}$ we sum the measured luminosities of the individual sources to obtain a more precise number. For the lower luminosities that contribute only a small fraction to the total luminosity we use the analytical description of the luminosity function given by Eqs. (3.10) and (3.12).

The integrated luminosity of HMXBs and LMXBs in the 2-10 keV ASM band calculated in such way are $\approx 2 \cdot 10^{38} \mathrm{erg} \mathrm{s}^{-1}$ and $\approx 2.5 \cdot 10^{39} \mathrm{erg} \mathrm{s}^{-1}$, respectively. Note that these numbers refer to the luminosity averaged over the period from 1996-2000. The variability of individual sources or an outburst of a bright transient can change the luminosity by a factor of up to $\sim 2-3$. Due to the shallow slopes of the luminosity functions the integrated X-ray emission of the Milky Way is dominated by the $\sim 5-10$ most luminous sources 


\begin{tabular}{|c|c|c|c|c|c|}
\hline \multirow[t]{2}{*}{ Source } & \multicolumn{3}{|c|}{$L_{X}\left[10^{38} \mathrm{erg} \mathrm{s}^{-1}\right]$} & \multirow{2}{*}{$\begin{array}{l}\text { dist. } \\
{[\mathrm{kpc}]}\end{array}$} & \multirow[t]{2}{*}{ Ref. } \\
\hline & avg. & $\min .^{(a)}$ & $\max .(a)$ & & \\
\hline Cir X-1 & 4.4 & 0.3 & 10 & 10.9 & 1 \\
\hline GRS $1915+105$ & 3.7 & 1 & 11 & 12.5 & 2 \\
\hline Sco X-1 & 2.7 & 2 & 4.5 & 2.8 & 3 \\
\hline Cyg X-2 & 1.8 & 0.9 & 3.4 & 11.3 & $1,4-7$ \\
\hline GX $349+2$ & 1.6 & 1.1 & 2.7 & 9.2 & $1,7,8$ \\
\hline GX $17+2$ & 1.5 & 1.1 & 2.4 & 9.5 & $\begin{array}{l}1,7,9 \\
10\end{array}$ \\
\hline GX 5-1 & 1.4 & 1 & 1.8 & 7.2 & 1,7 \\
\hline GX $340+0$ & 1.3 & 0.9 & 1.8 & 11.0 & 1,7 \\
\hline GX $9+1$ & 0.75 & 0.5 & 1.0 & 7.2 & 9 \\
\hline NGC 6624 & 0.47 & 0.15 & 0.8 & 8.0 & 10,11 \\
\hline Ser X-1 & 0.43 & 0.26 & 0.6 & 8.4 & 12 \\
\hline GX 13+1 & 0.41 & 0.25 & 0.6 & 7.0 & 13 \\
\hline X 1735-444 & 0.35 & 0.2 & 0.6 & 9.2 & 1 \\
\hline XTE J1550-564 & 0.35 & 0.005 & 2.1 & 5.3 & 14 \\
\hline KS 1731-260 & 0.28 & 0.06 & 0.6 & 8.5 & $15-17$ \\
\hline X 1705-440 & 0.25 & $<0.04$ & 0.6 & 7.4 & 18 \\
\hline X 1624-490 & 0.24 & $<0.13$ & 0.4 & 13.5 & 9 \\
\hline
\end{tabular}

TABLE 3.2.: List of the most luminous LMXB sources contributing $\approx 90 \%$ to the integrated luminosity of LMXBs in the $2-10 \mathrm{keV}$ band, averaged over 1996-2000. The 12 most luminous sources contribute $\approx 80 \%$ of the integrated luminosity.

(a) min. and max. luminosity were estimated by eye from the 1 day averaged light curves.

References for the distances: (1) - van Paradijs \& White (1995), (2) - Mirabel \& Rodriguez (1994), (3) - Bradshaw et al. (1999), (4) - Orosz \& Kuulkers (1999), (5) - Cowley et al. (1979), (6) - Smale (1998), (7) - Penninx (1989), (8) - Wachter \& Margon (1996), (9) - Christian \& Swank (1997), (10) - Djorgovski (1993), (11) - Webbink (1985), (12) - Ebisuzaki et al. (1984), (13) - Bandyopadhyay et al. (1999), (14) Orosz et al. (2002), (15) - Barret et al. (1998), (16) - Smith et al. (1997), (17) - Sunyaev (1990), (18) Haberl \& Titarchuk (1995)

(see Table 3.2.2 and 3.2.2). The maximum and minimum values for the luminosities were estimated by eye from the 1 day averaged light curves. The values in the tables therefore differ from the values in Table 3.2.3.

The majority of the brightest X-ray binaries are strongly variable on all time scales from milliseconds to years-tens of years. Therefore the luminosity of our Galaxy as a whole is also be subject to strong variability. This is important because with a powerful X-ray telescope such as XEUS it will be possible to detect X-ray flux from distant galaxies on the level of $L \sim 10^{40} \mathrm{erg} \mathrm{s}^{-1}$ but only short time scale variability would permit to distinguish the collective emission of X-ray binaries from the low luminosity, AGN-type activity of the nucleus. Black holes are unable to produce strong variability with characteristic times significantly shorter than a few 0.01s $\frac{M_{\mathrm{BH}}}{M_{\odot}}$ (Sunyaev \& Revnivtsev 2000). For super-massive black holes the characteristic time is of order or above $\sim 10^{3} \mathrm{~s}$.

Normalised to the star formation rate which is about $4 \mathrm{M}_{\odot} \mathrm{yr}^{-1}$ in the Milky Way $($ McKee \& Williams 1997) galactic HMXBs emit about $\sim 5 \cdot 10^{37} \mathrm{erg} \mathrm{s}^{-1} /\left(\mathrm{M}_{\odot} \mathrm{yr}^{-1}\right)$. The luminosity of LMXBs normalised to the stellar mass is about $\sim 5 \cdot 10^{28} \mathrm{erg} \mathrm{s}^{-1} \mathrm{M}_{\odot}^{-1}$, assuming a stellar 


\begin{tabular}{|c|c|c|c|c|c|}
\hline \multirow[t]{2}{*}{ Source } & \multicolumn{3}{|c|}{$L_{X}\left[10^{38} \mathrm{erg} \mathrm{s}^{-1}\right]$} & \multirow{2}{*}{$\begin{array}{l}\text { dist. } \\
{[\mathrm{kpc}]}\end{array}$} & \multirow[t]{2}{*}{ Ref. } \\
\hline & avg. & $\min .^{(a)}$ & $\max .{ }^{(a)}$ & & \\
\hline Cyg X-3 & 0.5 & 0.08 & 1.4 & 9.0 & 1 \\
\hline Cen X-3 & 0.15 & $<0.03$ & 0.7 & 9.0 & $2-5$ \\
\hline Cyg X-1 & 0.05 & 0.02 & 0.17 & 2.1 & 6 \\
\hline X $1657-415$ & 0.043 & $<0.02$ & 0.22 & 11.0 & 7 \\
\hline V4641 Sgr & 0.028 & $<0.02$ & 7.3 & 9.9 & 8 \\
\hline GX 301-2 & 0.02 & $<0.005$ & 0.4 & 5.3 & 9 \\
\hline XTE J1855-024 & 0.015 & $<0.01$ & 0.11 & 10.0 & 10 \\
\hline X1538-522 & 0.014 & $<0.008$ & 0.08 & 6.4 & 11 \\
\hline GS1843+009 & 0.01 & $<0.007$ & 0.11 & 10.0 & 12 \\
\hline $\mathrm{X} 1908+075$ & 0.008 & $<0.006$ & 0.05 & 6.4 & $\begin{array}{l}13 \\
14\end{array}$ \\
\hline
\end{tabular}

TABLE 3.3.: List of the most luminous HMXB sources that contribute $\approx$ $40 \%$ to the integrated luminosity of HMXBs in the 2-10 keV band, averaged over 1996-2000.

(a) min. and max. luminosity were estimated by eye from the 1 day averaged light curves.

References for the distances: (1) - Predehl et al. (2000), (2) - Krzeminski (1974), (3) - Hutchings et al. (1979), (4) - Motch et al. (1997),(5) - Bahcall (1978), (6) - Massey et al. (1995), (7) - Chakrabarty et al. (1993), (8) - Orosz et al. (2000), (9) - Kaper et al. (1995), (10) - Corbet et al. (1999), (11) - Reynolds et al. (1992), (12) - Israel et al. (2001), (13) - Wen et al. (2000), (14) - van Paradijs \& White (1995)

mass of the Galaxy of about $5 \cdot 10^{10} \mathrm{M}_{\odot}$.

The contribution of Be X-ray binaries from the ASM sample to the integrated luminosity of HMXBs is $\sim 5 \%$.

Note that poor knowledge of the shape of the luminosity function at low luminosities, $L \lesssim 10^{35} \mathrm{erg} \mathrm{s}^{-1}$ should not influence the total luminosity considerably unless the luminosity function steepens significantly at these low luminosities (see chapter 4.1).

The total number of X-ray binaries above $2 \cdot 10^{35} \mathrm{erg} \mathrm{s}^{-1}$ obtained from the luminosity functions is about $\sim 190$ of which $\sim 55$ are HMXBs and $\sim 135-$ LMXBs.

\subsubsection{Luminosity function and $\dot{M}$ distribution of X-ray binaries}

The X-ray luminosity function is obviously related to the distribution of X-ray binary systems over the mass loss rate of the secondary, $\dot{M}$. The simplest assumption would be that both distributions have the same slope in the range corresponding to luminosities of $\sim(0.01-1) L_{E d d}$. At larger luminosities, $L \gtrsim L_{E d d}$, the luminosity function has a break or cut-off, well in accordance with theoretical expectation, that the luminosity due to accretion cannot exceed the Eddington luminosity of the primary star by a large factor (see discussion in chapter 4.2). The donor star in a binary system, on the other hand, "does not know" about the Eddington critical luminosity, therefore the distribution of binary systems over the mass loss rate of the secondary, $\dot{M}$, is not expected to break near the Eddington value for the compact object. Thus the distribution of binary systems over $\dot{M}$ is expected to continue with the same slope well beyond the Eddington value.

Extremely super-Eddington values of the mass accretion rate $\dot{M}$ can result in quenching of the X-ray source and/or its obscuration by the matter expelled from the system by 
radiation pressure (Shakura \& Sunyaev 1973). This would lead to the appearance of a peculiar object, dim in X-rays and extremely bright in the optical and UV band - similar to SS 433 or the recent fast transient V4641 Sgr at the peak of its optical outburst. Such objects would emit only a negligible fraction in the X-ray band and would contribute to the lower luminosity end of the XRB luminosity function.

For moderately super-Eddington values of $\dot{M} \lesssim 10-100 \dot{M}_{E d d}$, however, one might expect the appearance of a near- or slightly super-Eddington source, therefore all such systems are expected to cluster near $L_{E d d}$, see Fig. 6.6 and the accompanying discussion. For a given slope of the luminosity function the number of such sources can be easily estimated. For the observed parameters of the LMXB luminosity function (slope $=1.3,42$ sources with $\left.36.5<\log \left(L_{X}\right)<38\right)$ and assuming that the $\dot{M}$ distribution continues with the same slope $=1.3$, the total number of sources with $\dot{M}$ corresponding to the range of luminosities of $10^{38}-10^{39}$ and $10^{39}-10^{40} \mathrm{erg} \mathrm{s}^{-1}$ is $\approx 10$ and $\approx 6$ correspondingly $(\approx 7$ sources are expected to have $\dot{M}$ corresponding to $L>10^{40} \mathrm{erg} \mathrm{s}^{-1}$ ). These estimates are in disagreement with the actually observed number of sources with $L \gtrsim 10^{38} \mathrm{erg} \mathrm{s}^{-1}$, which is equal to 8 . In order to reconcile the expected number of sources near $L_{E d d}$ with the observations, a slope of the $\dot{M}$ distribution of $\gtrsim 1.35-1.40$ is required which is somewhat steeper than the observed value of $\sim 1.3$. We note that the slope of $\sim 1.35$ is within $\sim 1 \sigma$ of the the observed value.

Finally, there are several effects that can suppress the number of the low luminosity sources, i.e. make the luminosity function flatter than the $\dot{M}$ distribution. The most obvious and important are discussed below.

- In the case of HMXBs the magnetosphere of the strongly magnetised, rapidly rotating neutron star can prevent the accretion at low $\dot{M}$ via the propeller effect (Illarionov \& Sunyaev 1975).

- Be-systems are characterised by regular outbursts corresponding to the passage of the neutron star through the equatorial stellar wind. Therefore for such sources the true value of the $\dot{M}$ in the binary system is measured by the peak luminosity during the outbursts whereas the long term averaged luminosity, used to construct the luminosity function, can give a significantly underestimated value.

- A common property of LMXBs, containing both neutron stars and black hole, is the presence of relativistic jets which might carry away a sizable fraction of the energy of accretion (Mirabel \& Rodríguez 1999). The presence of jets correlates with the X-ray spectral state: the jets are absent (and hence the true accretion efficiency is higher) in the soft spectral state corresponding to higher values of $\dot{M}$. The jets exist only in the hard spectral state (Fender 2001), thus decreasing the accretion efficiency at lower $\dot{M}$.

- In the case of black hole binaries an ADAF can form at low accretion rate in which case the accretion efficiency is proportional to $\dot{M}$ and the X-ray luminosity scales as $L \propto \dot{M}^{2}$ (Narayan \& Yi 1995). 
- At sufficiently low accretion rates a source becomes a transient with a recurrence time varying from $\sim 1$ to $\gtrsim 50$ years (White et al. 1984). This would decrease the number of low and intermediate luminosity sources in the luminosity function constructed on the several years baseline.

The number of luminous X-ray binaries in the Milky Way is insufficient to study the shape of the luminosity function near $L_{E d d}$ in detail. On the other hand within next several years CHANDRA X-ray observatory will study compact sources in a large number of nearby, $d \lesssim 20-30 \mathrm{Mpc}$ galaxies and the total number of the X-ray binaries detected in other galaxies can easily reach several hundred or thousand. In this context it might be interesting to construct a combined luminosity function of X-ray binaries in our and other galaxies to study its exact shape at the high luminosity end and search for a possible excess of sources near $L_{E d d}$. 


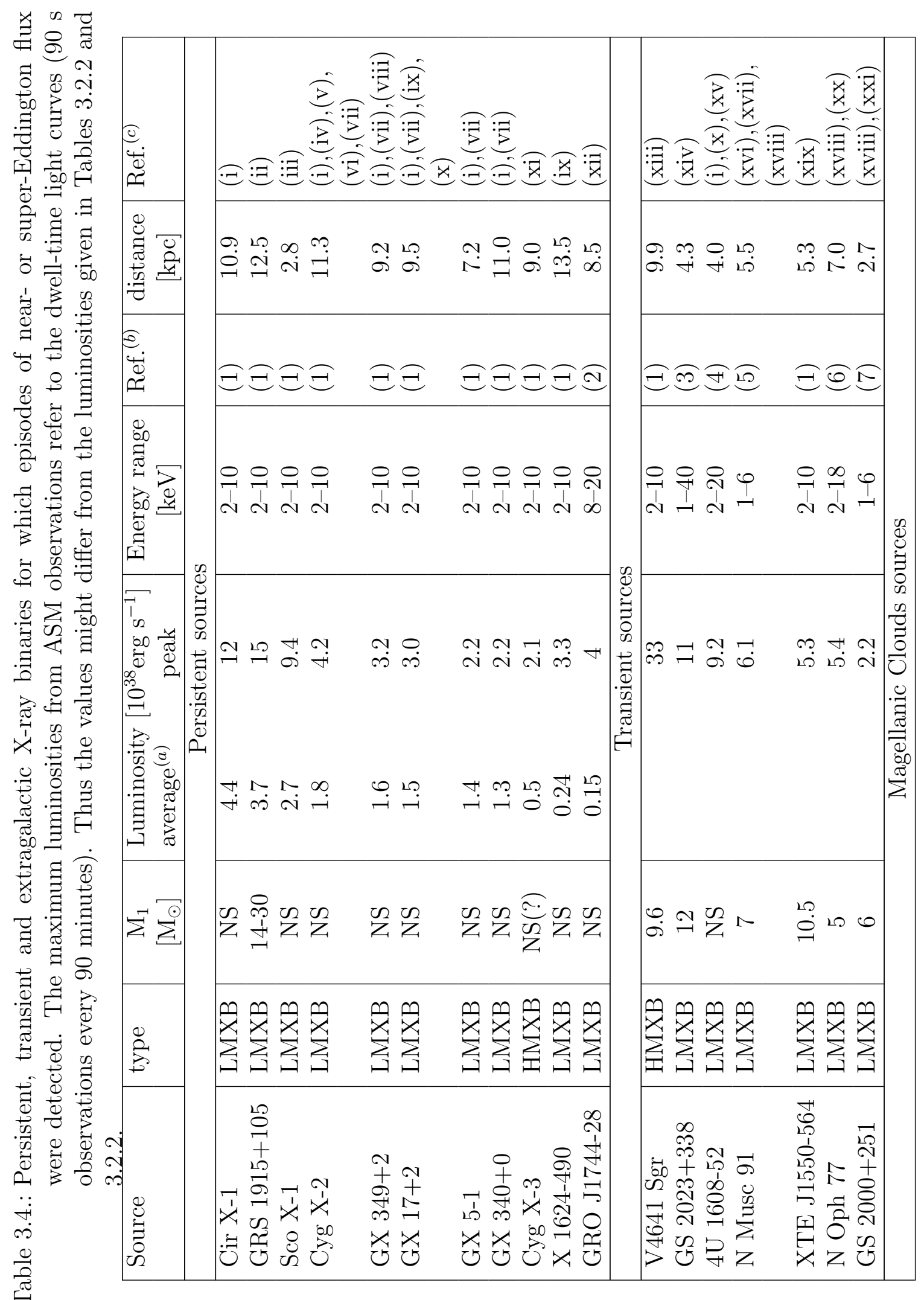



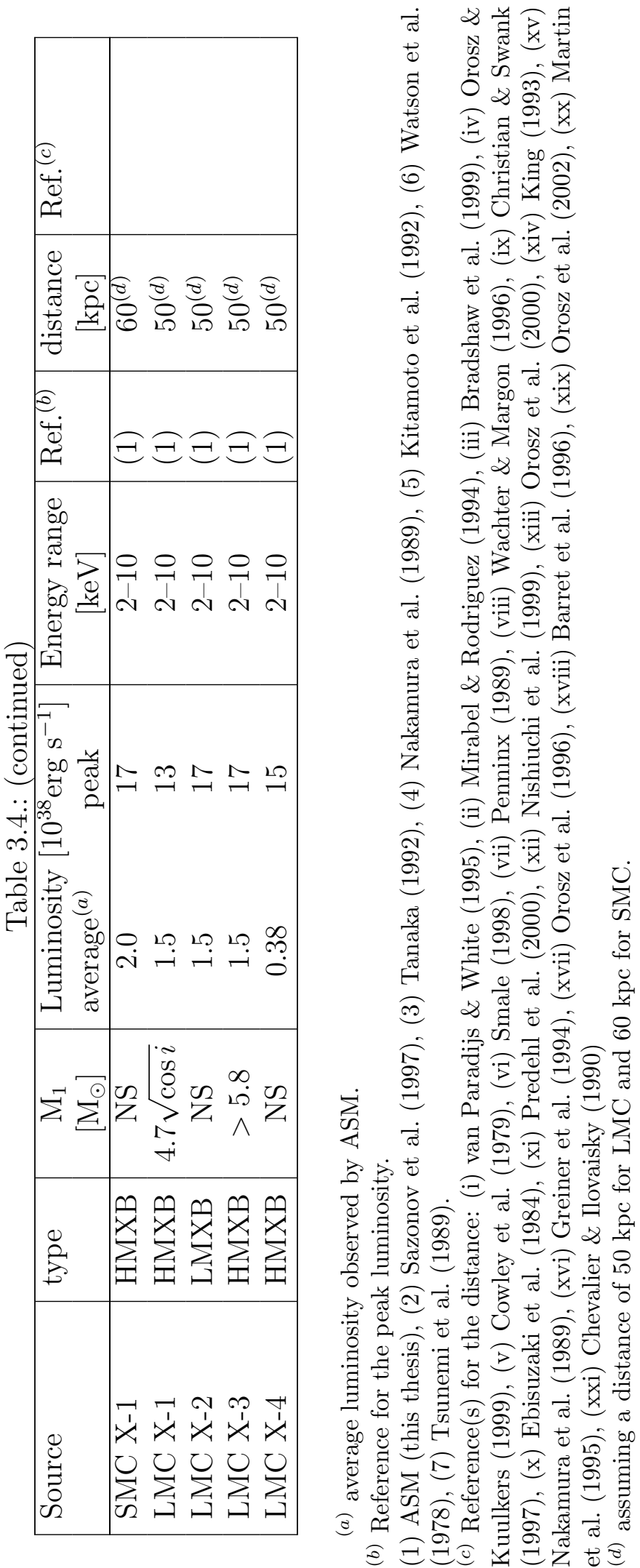


\section{Extension of luminosity functions}

\subsection{Low luminosity sources}

\subsubsection{Extension of $\log (\mathrm{N})-\log (\mathrm{S})$ towards lower fluxes}

Since the sensitivity of ASM is limited to relatively high flux sources it is interesting to investigate the behaviour of the $\log (\mathrm{N})-\log (\mathrm{S})$ at lower fluxes. Note that, given the slope observed by ASM (1.2 and 1.61 for LMXBs and HMXBs), the $\log (\mathrm{N})-\log (\mathrm{S})$ distribution should flatten at low fluxes since the total number of sources in the Galaxy is finite.

In order to study the low flux regime below the ASM completeness limit of $\approx 6.4 \cdot 10^{-11}$ erg $\mathrm{s}^{-1} \mathrm{~cm}^{-2}$, we use ASCA data from the Galactic Ridge Survey (Sugizaki et al. 2001) covering $\approx 40$ square degrees with the limiting sensitivity of $\sim 3 \cdot 10^{-13} \mathrm{erg} \mathrm{s}^{-1} \mathrm{~cm}^{-2}$. Since most of the sources in the ASCA survey are unidentified we followed the criterion suggested by Sugizaki et al. (2001) in order to discriminate X-ray binary candidates from other sources: that X-ray binary candidates have either a spectral photon index $\Gamma<1$, or a spectral photon index $\Gamma<3$ and a column density $N_{H}<0.8 \cdot 10^{22} \mathrm{~cm}^{-2}$. Excluding otherwise identified sources with these spectral properties there remain 28 sources. We fit the $\log (\mathrm{N})-\log (\mathrm{S})$ of the selected sources with the procedure similar to that used for ASM sources, modified to account for the flux dependent sky coverage of the ASCA survey (Fig. 7 in Sugizaki et al. (2001)). The resulting $\log (\mathrm{N})-\log (\mathrm{S})$ is:

$$
N(>S)=9.4 \cdot 10^{-5} \cdot S^{-0.42 \pm 0.08}
$$

where $S$ is flux in units of $10^{-12} \mathrm{erg} \mathrm{s}^{-1} \mathrm{~cm}^{-2}$. To compare ASCA data with an extrapolation of the ASM number-flux relation one needs to account for the difference in their sky coverage $\left(|l| \lesssim 40^{\circ}\right.$ and $|b| \lesssim 0.3^{\circ}$ for ASCA survey and entire sky for the ASM data). An approximate value of the correction factor can be estimated as the fraction of the mass of the Milky Way covered by the ASCA Galactic Ridge Survey with account for its sensitivity and the particular pattern of its sky coverage (Fig. 1 in Sugizaki et al. (2001)). The mass fraction was calculated using the Galaxy model described in chapter 3.1 and equals to $\sim$ 1:21. Converting the cumulative $\log (\mathrm{N})-\log (\mathrm{S})$ to differential $\log (\mathrm{N})-\log (\mathrm{S})$ for ASCA $\mathrm{X}$-ray binary candidates and all ASM X-ray binaries and multiplying the resulting ASCA $\log (\mathrm{N})-\log (\mathrm{S})$ by 21 we obtain the result shown in Fig. 4.1.

It is obvious that the agreement between ASM and ASCA data is sufficiently good. The slopes are different at the $\sim 2 \sigma$ level. On the other hand since the sources are all unidentified and their distances unknown it is not possible to distinguish between high and low mass X-ray binaries which have different slopes of their $\log (\mathrm{N})-\log (\mathrm{S})$ distributions 


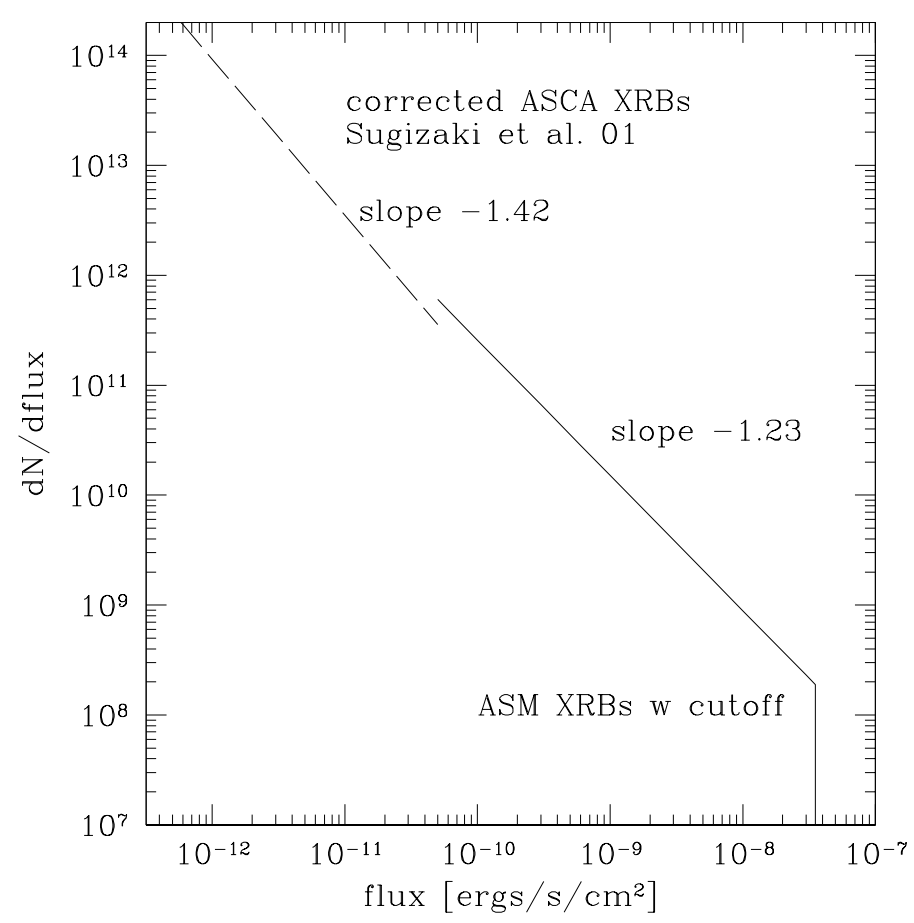

Figure 4.1.: Comparison of the differential $\log (\mathrm{N})-\log (\mathrm{S})$ relation for Galactic X-ray binaries obtained by ASM (solid line with break) and by ASCA Galactic Ridge Survey (dashed line). The ASCA numberflux relation was multiplied by an approximate correction factor accounting for the difference in the sky coverage of the ASM and ASCA surveys (see text for details).

in the ASM sample. Indeed, due to the small range in Galactic latitude $b^{I I}$ covered by the ASCA survey and due to the fact that HMXBs have a 3 times smaller vertical scale height (cf. chapter 3.1), the ratio of HMXBs to LMXBs should be different for the ASCA and ASM samples. The fraction of HMXBs, having steeper $\log (\mathrm{N})-\log (\mathrm{S})$, should be larger in the ASCA sample and thus the resulting $\log (\mathrm{N})-\log (\mathrm{S})$ should be somewhat steeper. We conclude that the data of the ASCA Galactic Ridge Survey indicate that there are no significant deviations in the $\log (\mathrm{N})-\log (\mathrm{S})$ from the extrapolations of the ASM data down to the sensitivity limit of the ASCA survey of $\sim 5 \cdot 10^{-13} \mathrm{erg} \mathrm{s}^{-1} \mathrm{~cm}^{-2}$.

\subsubsection{Low luminosity end of $\mathrm{X}$-ray binary luminosity function}

Knowledge of the $\log (\mathrm{N})-\log (\mathrm{S})$ observed by ASCA and the spatial distribution of sources in the Galaxy gives a possibility to constrain the low luminosity end of the luminosity function. If the luminosity function observed with ASM continues to lower luminosities then it should be possible to reproduce the $\log (\mathrm{N})-\log (\mathrm{S})$ observed by ASCA according to the formula

$$
N(>S)=\int_{L_{\min }}^{L_{\max }} \frac{d N}{d L} \cdot \frac{M(<r)_{\mathrm{ASCA}}}{M_{\text {total }}} d L,
$$

with

$$
r=\sqrt{\frac{L}{4 \pi \cdot S}}
$$


where $N(>S)$ is the number of sources with a flux higher than $S$ observed by ASCA, $\frac{d N}{d L}$ is the differential luminosity function, and $\frac{M(<r)_{\mathrm{ASCA}}}{M_{\text {total }}}$ is the fraction of mass within a radius $r$ from the Earth within the field of view of the ASCA survey, $L_{\max }$ is the high luminosity cut-off of the luminosity function (Eqs. (3.10) and (3.12)). The $L_{\min }$ is the low luminosity cut-off of the luminosity function below which it is assumed to be equal to zero. This quantity characterises roughly the luminosity level at which the luminosity function deviates significantly from the extrapolation of the ASM power law.

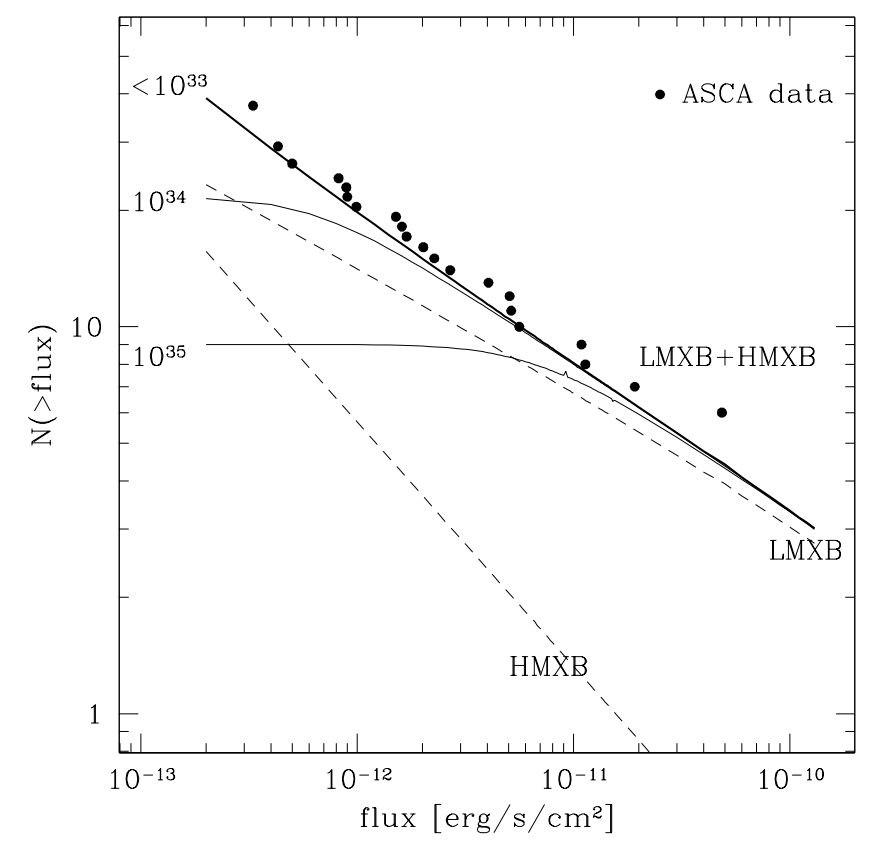

Figure 4.2.: Comparison of the number-flux relation observed in the ASCA Galactic Ridge Survey (points) and the predicted number-flux relation based on the extrapolation of the ASM luminosity function to low luminosities (lines). The vertical axis shows the number of sources in the entire field of the ASCA survey. The ASCA number-flux relation was corrected for the flux dependent sky coverage (Fig. 7 in Sugizaki et al. (2001)). The predicted number-flux relations were computed according to Eq. (4.2) using the extrapolation of the ASM luminosity functions and the volume density distributions of $\mathrm{X}$-ray binaries described in chapter 3.1. The thick solid lines show the combined $\log (\mathrm{N})-\log (\mathrm{S})$ of LMXBs and HMXBs for different values of the low luminosity cut-off. The thin dashed lines show the contributions of LMXBs and HMXBs separately for the case without cut-off.

The predicted $\log (\mathrm{N})-\log (\mathrm{S})$ calculated from Eq. (4.2) is compared with the $\log (\mathrm{N})-$ $\log (\mathrm{S})$ of X-ray binary candidates from the ASCA survey in Fig. 4.2. In plotting the ASCA data (solid circles) we added five bright sources located in the ASCA field of view that were excluded from the final catalogue in Sugizaki et al. (2001) and corrected for the flux dependent sky coverage of the ASCA survey (Fig. 7 in Sugizaki et al. (2001)). The predicted $\log (\mathrm{N})-\log (\mathrm{S})$ was calculated according to Eq. (4.2) separately for HMXB and LMXB using the extrapolation of the respective ASM luminosity functions. The mass integral $M(<r)$ in Eq. (4.2) was calculated taking approximately into account the actual pattern of ASCA pointings and using the volume density distributions constructed in chapter 3.1. The predicted combined $\log (\mathrm{N})-\log (\mathrm{S})$ of HMXB and LMXB sources is shown in Fig. 4.2 by the thick solid lines for different values of the low luminosity cut-off 
$L_{\min }$. The thin solid and dashed lines show the contributions of HMXBs and LMXBs respectively for the case without low luminosity cut-off.

It is clear from Fig. 4.2 that the predicted number-flux relation of X-ray binaries agrees with the ASCA data very well. Given the volume density distributions of X-ray binaries in the Galaxy, the low flux end of the ASCA $\log (\mathrm{N})-\log (\mathrm{S})$ is sensitive to sources with luminosities of $\sim 10^{34} \mathrm{erg} \mathrm{s}^{-1}$. The good agreement with the predicted $\log (\mathrm{N})-\log (\mathrm{S})$ distribution implies that the data do not require a low luminosity cut-off of the luminosity function down to $\sim 10^{34} \mathrm{erg} \mathrm{s}^{-1}$.

\subsubsection{Young objects in star forming regions}

Recent observations with the CHANDRA X-ray observatory of the Orion Nebula cluster allow one to estimate the contribution to the X-ray emission from young objects in the star forming regions. Schulz et al. (2001) observed the Orion Trapezium region and found 111 sources above the sensitivity threshold of $6.6 \cdot 10^{28} \mathrm{erg} \mathrm{s}^{-1}$, assuming a distance of 440 pc. The total luminosity of their sample is about $5.6 \cdot 10^{32} \mathrm{erg} \mathrm{s}^{-1}$. This luminosity is dominated by the brightest source in the Orion Nebula cluster, $\theta^{1}$ Ori C, which provides about $1.8 \cdot 10^{32} \mathrm{erg} \mathrm{s}^{-1}$. Extrapolating this result to the whole Orion Nebula Cluster in which CHANDRA observed about 1000 sources we obtain a total luminosity of the star cluster of about $4 \cdot 10^{33} \mathrm{erg} \mathrm{s}^{-1}$, counting the luminosity of $\theta^{1}$ Ori $\mathrm{C}$ only once and multiplying the rest by 10, assuming the luminosity function of the Trapezium region is representative for the whole Orion Nebula cluster. To estimate the X-ray luminosity of all star forming regions in the Galaxy one can proceed in two ways. Taking the mass of the molecular gas in the Orion cluster to be $\sim 10^{5} \mathrm{M}_{\odot}$ (Maddalena et al. 1986), and the total mass of the molecular gas in the Galaxy to be $\sim 10^{9} \mathrm{M}_{\odot}$ (Williams \& McKee 1997), the total luminosity is $\sim 4 \cdot 10^{37} \mathrm{erg} \mathrm{s}^{-1}$. On the other hand one can use the star formation rate in the Orion Nebula cluster and the Galaxy as the determining factor. Taking the total SFR in Orion to be $\geq 10^{-4} \mathrm{M}_{\odot} \mathrm{yr}^{-1}$ (Hillenbrand 1997), and the total SFR in the Galaxy to be $4 \mathrm{M}_{\odot} \mathrm{yr}^{-1}$ (McKee \& Williams 1997), the total luminosity of young objects in the star forming regions in the Galaxy is $\lesssim 1.6 \cdot 10^{38} \mathrm{erg} \mathrm{s}^{-1}$. Taking into account that the latter value is an upper limit, both numbers agree sufficiently well. Therefore star forming regions contribute less than $\sim$ few per cent to the integrated X-ray emission of the Galaxy but $\sim 20 \%$ or more to the luminosity of HMXBs in the energy range from $2-10 \mathrm{keV}$. On the other hand the spectrum of young stellar objects is much softer than the spectrum of $\mathrm{X}$-ray binaries.

\subsection{High luminosity sources}

In recent months the CHANDRA X-ray observatory was able to resolve single X-ray sources in other galaxies that appear to radiate at or above the Eddington limit for a $1.4 \mathrm{M}_{\odot}$ neutron star, i.e. $\sim 2 \cdot 10^{38} \mathrm{erg} \mathrm{s}^{-1}$. Similar behaviour is also observed in Galactic X-ray binaries by ASM. The slightly different spectral band used in these CHANDRA obser- 
vations, usually $0.3-10 \mathrm{keV}$ compared to $2-10 \mathrm{keV}$ for ASM, does not lead to significant differences in luminosity.

Table 3.2.3 lists the sources which were observed either by ASM or some other instrument to emit at or above the Eddington limit for a $1.4 \mathrm{M}_{\odot}$ neutron star. The spatial distribution of these sources is shown in Fig. 4.3 and can be compared to the distributions of the brightest sources observed by CHANDRA in other galaxies.
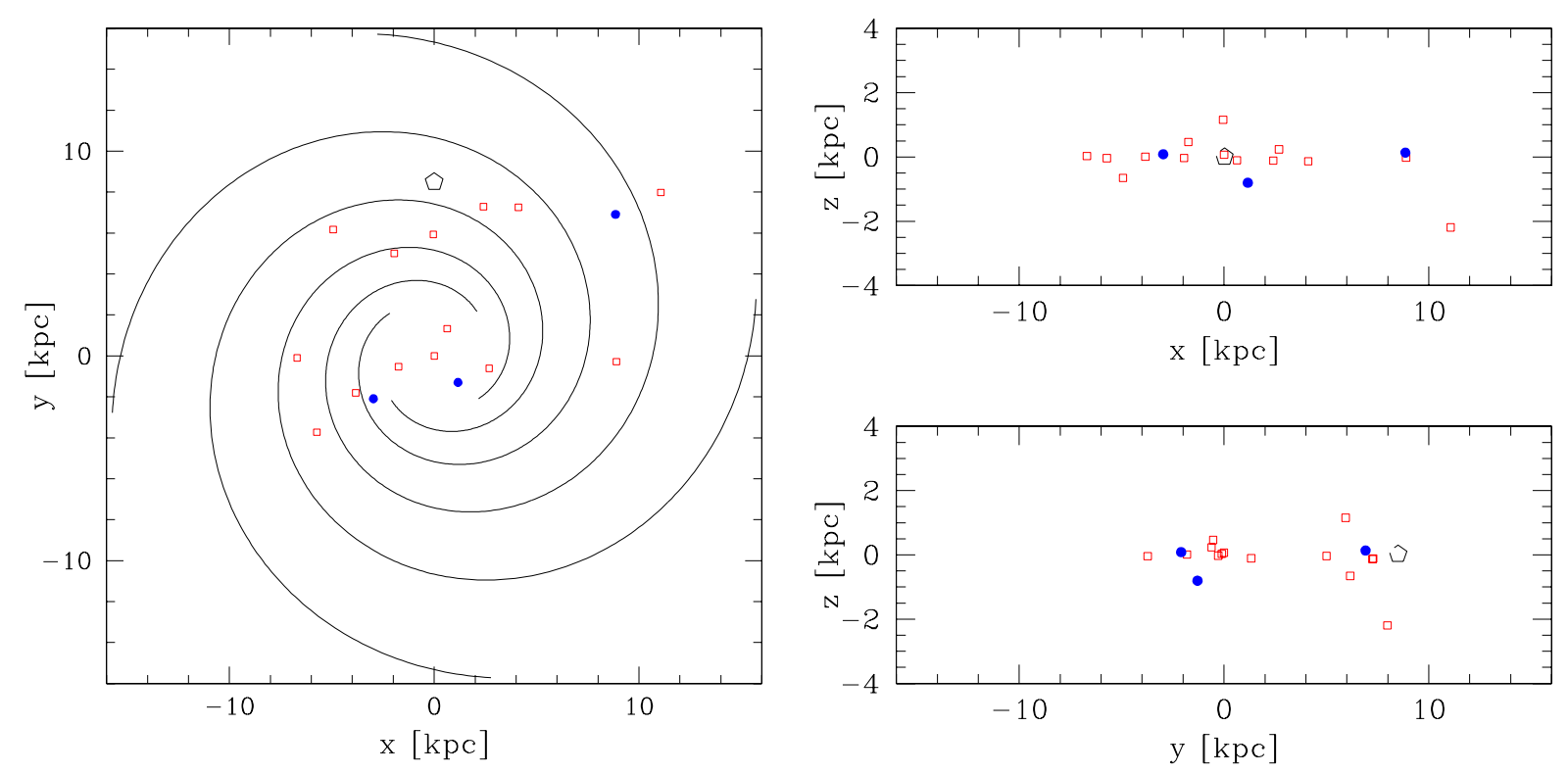

FiguRE 4.3.: The spatial distribution of Galactic X-ray binaries that have shown episodes of Eddington or super-Eddington luminosity for a $1.4 \mathrm{M}_{\odot}$ neutron star. The coordinate system is the same as in Fig. 3.2. Filled circles indicate HMXBs, open squares indicate LMXBs. Note that fact that the majority of the sources are located at $y>0$ reflects the flux limited nature of the ASM sample.

There are several reasons why sources can emit super-Eddington luminosity:

- For accreting black holes in high state radiation is coming from the quasi-flat accretion disk where electron scattering gives the main contribution to the opacity. Under these conditions the radiation is emitted according to

$$
f(\mu)=(1+2.08 \mu) \mu
$$

where $\mu=\cos (i)$ where $i$ is the inclination angle. It is easy to show that the radiation flux perpendicular to the plane of the disk exceeds the average value by 3 times (see Shakura \& Sunyaev (1973) for discussion).

- Some of the normal stars entering the X-ray binary phase are strongly evolved and have an unusual chemical abundance, e.g. if a He-enriched star supplies matter the 
Eddington luminosity is twice higher than for hydrogen plasma due to the change in cross-section per nucleus.

Just these two factors permit to surpass the classical Eddington limit by a factor of $\sim 6$.

- The star supplying material to the neutron star or black hole "does not know" about the existence of the Eddington luminosity limit due to accretion. Therefore some part of the matter will outflow forming a supercritical disk. In the approach of Shakura \& Sunyaev (1973) it is possible to gain a factor of $\ln (\dot{m}) \approx 3-5$ for $\dot{m}>>1$ with $\dot{m}=\frac{\dot{M}}{M_{E d d}}$. Paczynsky \& Wiita (1980) and Abramowicz et al. (1988) constructed the solution of slim disks which also permits luminosities higher than the Eddington luminosity.

- Many X-ray binaries show from time to time the acceleration of powerful jets (Mirabel \& Rodríguez 1999). These relativistic jets might produce strongly beamed X-ray emission with flux strongly exceeding the average and Eddington critical value for isotropic sources. See also the discussion by Koerding et al. (2001), Fabrika \& Mescheryakov (2000) and King et al. (2001).

- In the case of accretion on to a neutron star with strong magnetic field the accretion columns form near the surface of the neutron star in the polar regions. Such columns can have a super-Eddington luminosity, because photons are emitted perpendicular to the axis of the accretion column and the light pressure force is balanced by magnetic field (Basko \& Sunyaev 1976).

- In Z-sources (luminous accreting neutron stars with low magnetic field) the boundary layer width expands rapidly with increasing accretion rate reaching several star radii (Popham \& Sunyaev 2001). This quasi-flat continuation of accretion disk might also have super-Eddington luminosity of the type of the slim disk. 


\section{Part II.}

\section{Star-forming galaxies}





\section{Data on star forming galaxies}

\subsection{Sample of galaxies}

The list of galaxies used in the following analysis is given in Table 5.1 and Table 5.2 along with their Hubble type, distances and other relevant parameters.

As our primary sample of local galaxies, used to study the HMXB luminosity function and to calibrate the $L_{X}$-SFR relation, we chose a number of nearby late-type/star-burst galaxies observed by CHANDRA. We based our selection primarily on two criteria. Firstly, we selected galaxies that can be spatially resolved by CHANDRA sufficiently well so that the contribution of a central AGN can be discriminated and the luminosity functions of the compact sources can be constructed without severe confusion effects. We should note, however, that for the most distant galaxies from our primary sample (e.g. NGC 3256), the probability of source confusion might become non-negligible. Secondly, we limited our sample to galaxies known to have high star formation rates, so that the population of X-ray binaries is dominated by HMXBs and the contribution of low mass X-ray binaries can be safely ignored (see sub-chapter 5.1.5 for more detailed discussion).

In order to probe the HMXB luminosity function in the low SFR regime, we used the results of the X-ray binary population study in the Milky Way, chapters 2- 4, based on RXTE/ASM observations and the luminosity function of high mass X-ray binaries in the Small Magellanic Cloud obtained by ASCA (Yokogawa et al. 2000).

The galaxies from our primary sample are listed in Table 5.1.

In addition, in order to increase the local sample, we selected galaxies observed by other $\mathrm{X}$-ray missions, mainly ASCA, for which no luminosity function is available, but a total flux measurement. The selection was based on the requirement that no AGN-related activity had been detected and the SFR to total mass ratio is sufficiently high to neglect the LMXB contribution. These galaxies were used to complement the primary sample in the analysis of the $L_{X}-$ SFR relation. They are listed in Table 5.2.

Finally, in order to study the $L_{X}$-SFR relation in distant galaxies at redshifts of $z \sim$ $0.2-1.3$ we used a number of galaxies detected by CHANDRA in the Hubble Deep Field North, see Table 6.1. The selection criteria are similar to those applied to the local sample and are described in more detail in Sec. 6.6. 

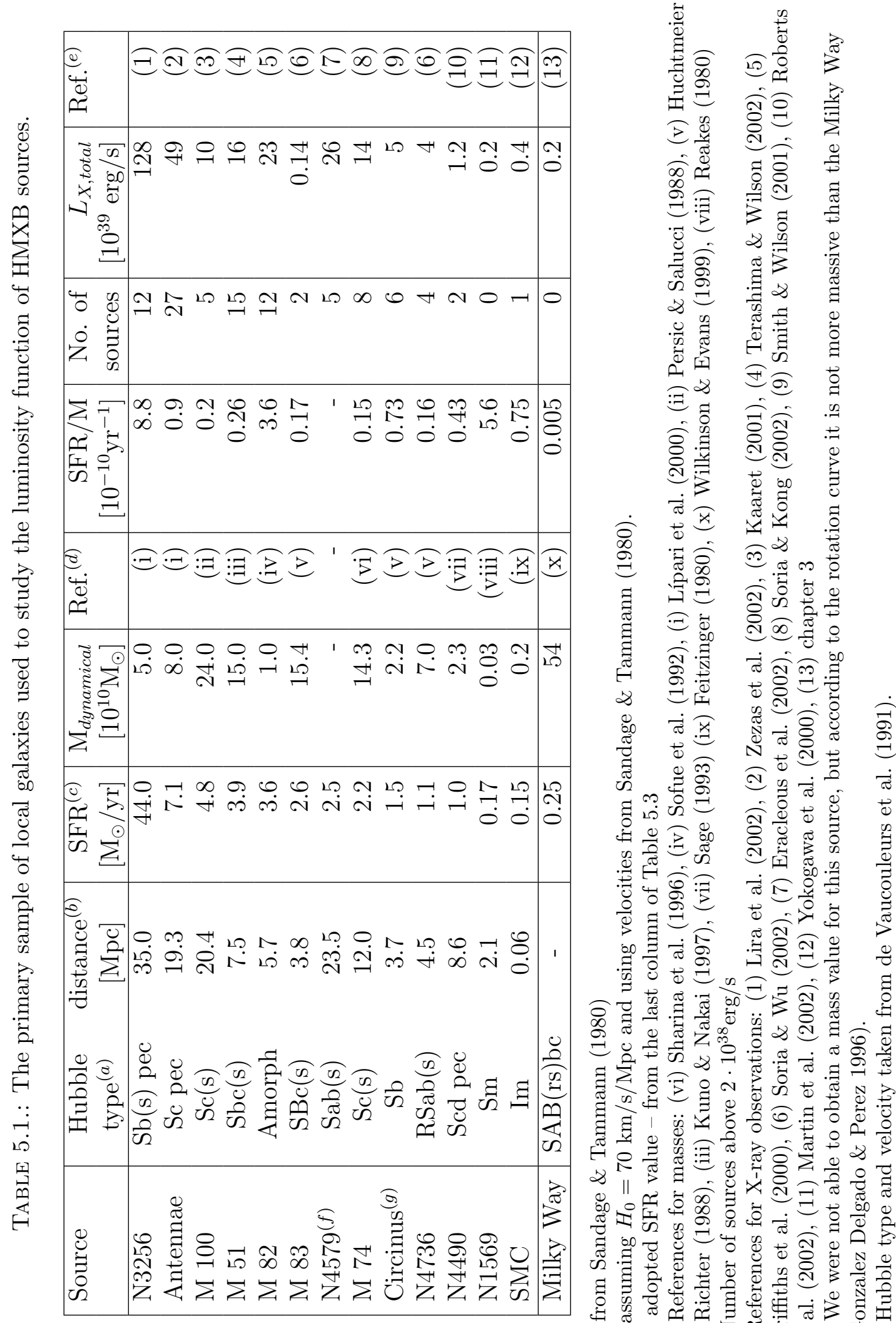

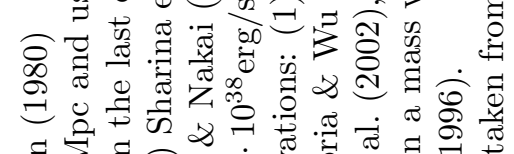

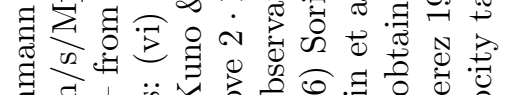
द्वे

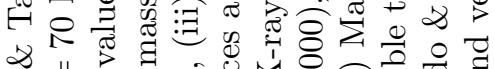

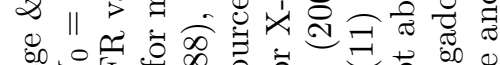

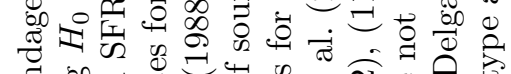

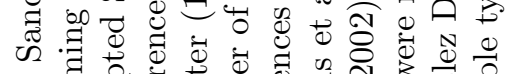

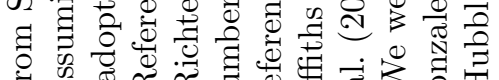

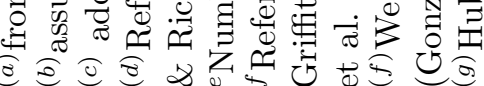




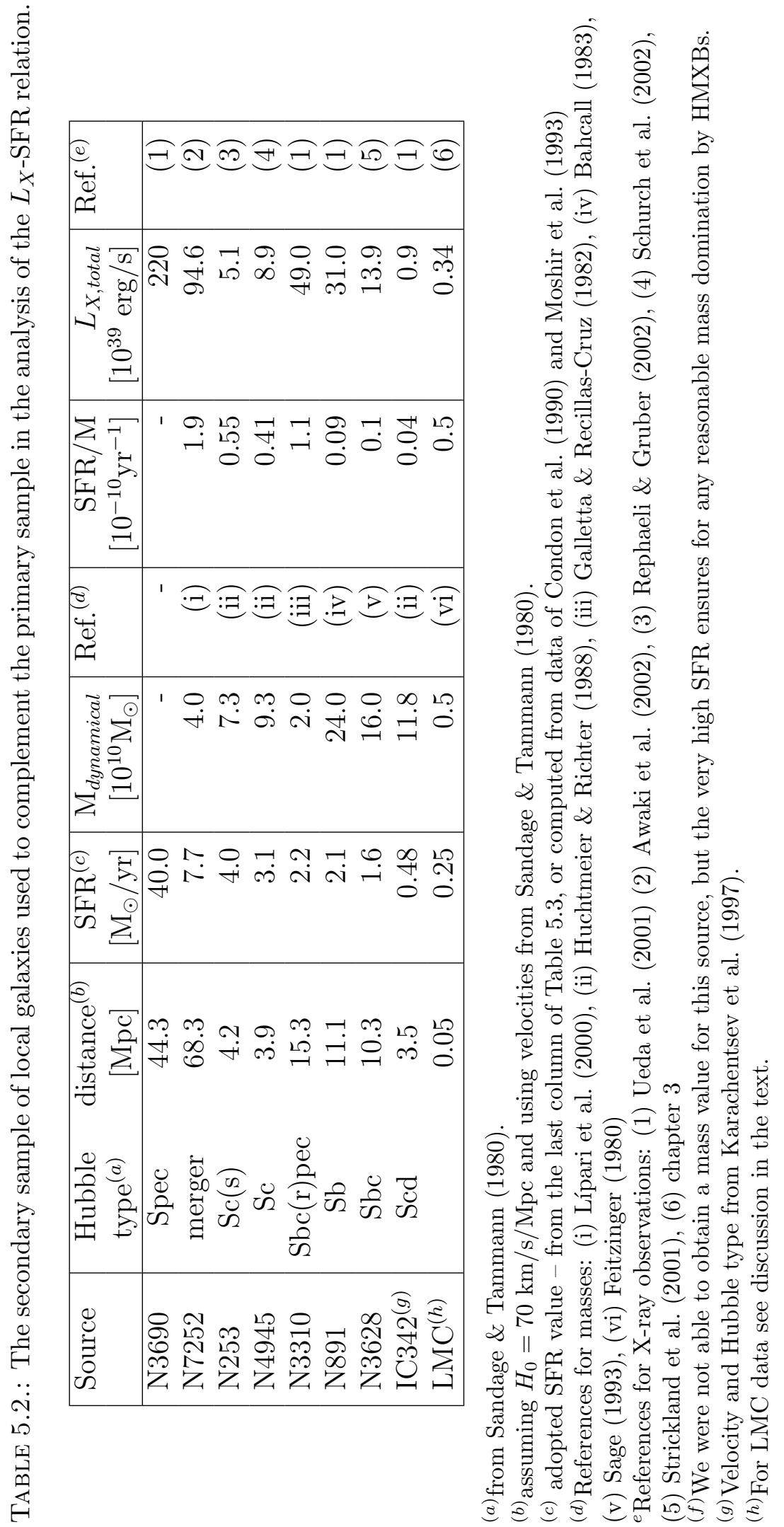




\subsubsection{Distances}

To estimate X-ray luminosity and star formation rate, which is also based on flux measurements in different spectral bands, and compare these values for different galaxies it is necessary to have a consistent set of distances. For the galaxies from our sample, given in Tables 5.1 and 5.2 cosmological effects are not important. The distances were calculated using velocities from Sandage \& Tammann (1980) corrected to the centre of mass of the Local Group and assuming a Hubble constant value of $H_{0}=70 \mathrm{~km} / \mathrm{s} / \mathrm{Mpc}$. The distances are listed in Table 5.1 and Table 5.2. Note that these distances might differ from the values used in the original publications on the X-ray luminosity functions and SFRs.

\subsubsection{X-ray luminosity functions}

For the X-ray luminosity functions we used published results of CHANDRA observations of late-type/star-burst galaxies. References to the original publications are given in Table 5.1 and Table 5.2. The luminosities were rescaled to the distances described in the previous sub-chapter. Note that, due to this correction, the total X-ray luminosities and luminosities of the brightest sources might differ from the numbers given in the original publications. The complete set of luminosity functions for all objects from the primary sample (Table 5.1) is plotted in Fig. 5.1 (left panel).

One of the most serious issues important for the following analysis is the completeness level of the luminosity functions which is obviously different for different galaxies, due to different exposure times and distances. In those cases when the completeness luminosity was not given in the original publication, we used a conservative estimate based on the luminosity at which the luminosity function starts to flatten.

Due to the relatively small field of view of CHANDRA and sufficiently high concentration of X-ray binaries in the central parts of the galaxies the contribution of foreground and background objects can be neglected for the purpose of our analysis (e.g. M 51 (Terashima \& Wilson 2002), M 83 (Soria \& Wu 2002)). Two of the galaxies in our sample - Circinus and NGC 3256 - are located at a Galactic latitude of $\left|b_{I I}\right|<20$. In these cases the contribution of foreground optical stars in the Galaxy that are bright in X-rays can be discriminated based on the softness of their spectra. Extrapolating the luminosity function of X-ray binaries in the Milky Way, chapter 5.1.2, the probability can be estimated of occurrence of a foreground source due to an unknown Galactic X-ray binary with a flux exceeding the sensitivity limit of the corresponding CHANDRA observations. For the CHANDRA field of view this probability is less than $\sim 10^{-3}$ and therefore can be neglected.

The luminosities of the compact sources were derived in the original publications in slightly different energy bands, under different assumptions about spectral shape, and with different absorption column densities. Although all these assumptions affect the luminosity estimates, the resulting uncertainty is significantly smaller than those due to distance uncertainty and uncertainties in the star formation rate estimates. Moreover, in many cases, due to insufficient statistics of the data an attempt to do corrections for these effects could result in additional uncertainties, larger than those due to a small difference 

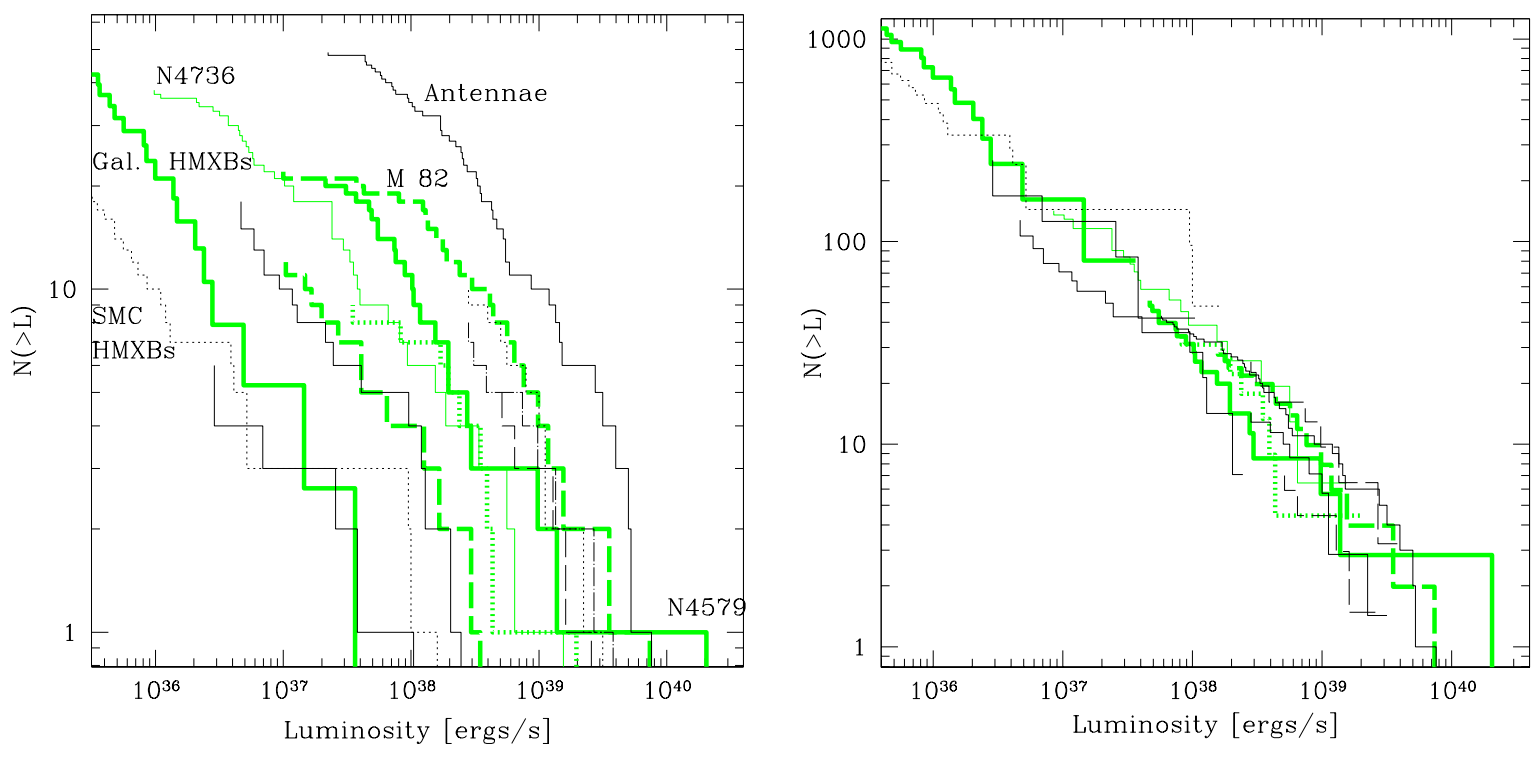

Figure 5.1.: Left: The luminosity functions of compact X-ray sources in nearby galaxies from the primary sample obtained by CHANDRA and listed in Table 5.1. The luminosity functions are plotted assuming the distances from Table 5.1. Right: The luminosity functions for the same galaxies scaled by the ratio of their star formation rate to the SFR of Antennae. The luminosity functions in the right panel are plotted only above their corresponding completeness limits. It is clear that despite large differences in the SFRs (by a factor of $\sim 40-50$ ) the scaled luminosity functions occupy only a narrow band in the $N(>L)-L$ plane.

in e.g. energy bands. Therefore we make no attempt to correct for these differences. It should be mentioned however, that the most serious effect, up to a factor of a few in luminosity might be connected with intrinsic absorption for the sources embedded in dense star-forming regions (Zezas et al. 2002). Appropriately accounting for this requires information about these sources, which is presently not available.

All the luminosity functions with exception of the Milky Way are "snapshots" of the duration of several tens of kilo-seconds. On the other hand, similar to the Milky Way, compact sources in other galaxies are known to be variable. E.g. NGC 3628 is dominated by a single source, that is known to vary by about a factor of 30 (Strickland et al. 2001). This may affect the shape of the individual luminosity functions. It should not however affect our conclusions, since in the high SFR regime they are based on the average properties of sufficiently many galaxies. As for the low SFR regime, the Milky Way data are an average of the RXTE/ASM observations over four years therefore the contribution of "standard" Galactic transient sources is averaged out. 


\subsubsection{Star formation rate estimates}

One of the main uncertainties involved is related to the SFR estimates. The conventional SFR indicators rely on a number of assumptions regarding the environment in a galaxy, such as dust content of the galaxy, or the shape of the initial mass function (IMF). Although comparative analysis of different star formation indicators is beyond the scope of this thesis, in order to roughly assess the amplitude of the uncertainties in the SFR estimates we compared results of different star formation indicators for each galaxy from our sample with special attention given to the galaxies from the primary sample. For all galaxies from the primary sample we found at least 3 different measurements of star formation indicators in the literature, namely $\mathrm{UV}, \mathrm{H}_{\alpha}$, FIR or thermal radio emission flux. The data along with the corresponding references are listed in Table 5.3.

In order to convert the flux measurements to star formation rates we use the result of an empirical cross-calibration of star formation rate indicators by Rosa-González et al. (2002). The calibration is based on the canonical formulae by Kennicutt (1998) and takes into account dust/extinction effects. We used the following flux-SFR relations:

$$
\begin{gathered}
S F R_{H_{\alpha}}=1.1 \cdot 10^{-41} \cdot L_{H_{\alpha}}[\mathrm{erg} / \mathrm{s}] \\
S F R_{U V}=6.4 \cdot 10^{-28} \cdot L_{U V}[\mathrm{erg} / \mathrm{s} / \mathrm{Hz}] \\
S F R_{F I R}=4.5 \cdot 10^{-44} \cdot L_{F I R}[\mathrm{erg} / \mathrm{s}] \\
S F R_{\text {radio }}=1.82 \cdot 10^{-28} \cdot \nu_{\mathrm{GHz}}^{0.1} \cdot L_{\nu}[\mathrm{erg} / \mathrm{s} / \mathrm{Hz}]
\end{gathered}
$$

The last relation is from Condon (1992) and applies only to the thermal radio emission, originating, presumably, in hot gas in HII regions associated with star formation (as we used thermal 1.4 GHz flux estimates from Bell \& Kennicutt (2001)).

The above relations refer to the SFR for stars more massive than $\sim 5 \mathrm{M}_{\odot}$. The total star formation rate, including low mass stars, could theoretically be obtained by extrapolating these numbers assuming an initial mass function. Obviously, such a correction would rely on the assumption that the IMF does not depend on the initial conditions in a galaxy and would involve a significant additional uncertainty. On the other hand, this correction is not needed for our study as the binary X-ray sources harbour a compact object - a NS or a $\mathrm{BH}$ - which according to the modern picture of stellar evolution can evolve only from stars with initial masses exceeding $\sim 8 \mathrm{M}_{\odot}$. The SFR correction from $M>5 \mathrm{M}_{\odot}$ to $M>8$ $\mathrm{M}_{\odot}$ is relatively small $(\sim 20 \%)$ and, most importantly, due to the similarity of the IMFs for large masses it is significantly less subject to the uncertainty due to poor knowledge of the slope of the IMF. Thus, for the purpose of our study it is entirely sufficient to use the relations (5.1)-(5.4) without an additional correction. In the following, the term SFR refers to the star formation rate of stars more massive than $\sim 5 \mathrm{M}_{\odot}$.

Since the relations (5.1)-(5.4) are based on the average properties of star forming galaxies there is considerable scatter in the SFR estimates of a galaxy obtained using different indicators (Table 5.3). On the other hand, the SFR estimates based on different measurements of the same indicator are generally in a good agreement with each other. A detailed study, which SFR indicator is most appropriate for a given galaxy is beyond the scope of 
this thesis. Therefore, we relied on the fact that for all galaxies from our primary sample there are more than 3 measurements for different indicators.

Table 5.3.: Star formation rates for galaxies of the local sample from different indicators.

\begin{tabular}{|c|c|c|c|c|c|c|c|c|c|c|}
\hline \multirow[t]{2}{*}{ Source } & \multicolumn{4}{|c|}{ Fluxes } & \multirow[b]{2}{*}{ Ref. } & \multicolumn{4}{|c|}{ SFRs } & \multirow{2}{*}{$\begin{array}{l}\text { adpt. } \\
\text { SFR }\end{array}$} \\
\hline & $\mathrm{UV}^{1}$ & $\mathrm{H}_{\alpha}{ }^{2}$ & $\mathrm{FIR}^{3}$ & $\operatorname{radio}^{4}$ & & UV & $\mathrm{H}_{\alpha}$ & FIR & radio & \\
\hline \multirow[t]{3}{*}{ N3256 } & & 0.33 & 4.68 & & (a) & & 5.3 & $\overline{31.0}$ & & \\
\hline & & & 7.1 & & (b) & & & 47.0 & & \\
\hline & & & 8.2 & & (c) & & & 54.0 & & 44 \\
\hline \multirow{3}{*}{$\begin{array}{l}\text { N4038/9 } \\
\text { (Antennae) }\end{array}$} & & 1.62 & & & (d) & & 7.9 & & & \\
\hline & 3.22 & 1.36 & 4.50 & 10.90 & (e) & 9.2 & 6.7 & 9.0 & 9.1 & \\
\hline & & & 2.30 & & (f) & & & 4.6 & & 7.1 \\
\hline \multirow[t]{3}{*}{ M 100} & & 0.81 & & & (d) & & 4.5 & & & \\
\hline & 3.07 & 0.72 & 3.36 & & (e) & 9.8 & 3.9 & 7.5 & & \\
\hline & & & 1.48 & & (f) & & & 3.3 & & 4.8 \\
\hline \multirow[t]{3}{*}{ M 51} & 15.4 & 3.45 & 14.7 & 8.62 & (e) & 6.6 & 2.6 & 4.5 & 1.1 & \\
\hline & & 4.68 & & & (d) & & 3.5 & & & \\
\hline & & 2.81 & & & (g) & & 2.1 & & & 3.9 \\
\hline \multirow[t]{4}{*}{ M 82} & & 6.17 & 52.0 & & (h) & & 2.6 & 9.1 & & \\
\hline & & 9.12 & & & (d) & & 3.9 & & & \\
\hline & 1.46 & 9.98 & 112.0 & 76.70 & (e) & 0.4 & 4.3 & 19.6 & 5.6 & \\
\hline & & & 53.0 & & (f) & & & 9.2 & & 3.6 \\
\hline \multirow[t]{3}{*}{ M 83} & & 13.50 & & & (i) & & 2.6 & & & \\
\hline & & 0.45 & & & (j) & & 0.1 & & & \\
\hline & 32.4 & 12.20 & 34.2 & & (e) & 3.6 & 2.3 & 2.7 & & 2.6 \\
\hline \multirow[t]{3}{*}{ N4579 } & & 0.36 & & & (i) & & 2.6 & & & \\
\hline & & 0.32 & & & (d) & & 2.4 & & & \\
\hline & & & 0.43 & & (f) & & & 1.3 & & 2.5 \\
\hline \multirow[t]{4}{*}{ M 74} & & 1.23 & & & (d) & & 2.3 & & & \\
\hline & 6.85 & 1.25 & 2.92 & & (e) & 7.6 & 2.4 & 2.3 & & \\
\hline & & 1.51 & & & (g) & & 2.9 & & & \\
\hline & & & 1.59 & & (f) & & & 1.2 & & 2.2 \\
\hline \multirow[t]{2}{*}{ Circinus } & & & 22.3 & & (c) & & & 1.6 & & \\
\hline & & 9.5 & 16.5 & & (k) & & 1.7 & 1.2 & & 1.5 \\
\hline \multirow[t]{3}{*}{ N4736 } & & 5.37 & & & (d) & & 1.6 & & & \\
\hline & & 5.37 & & & (i) & & 1.6 & & & \\
\hline & 6.49 & 2.10 & 6.78 & 5.80 & (e) & 1.1 & 0.6 & 0.8 & 0.3 & 1.1 \\
\hline \multirow[t]{3}{*}{ N4490 } & & 1.10 & 4.42 & & $(1)$ & & 1.1 & 1.8 & & \\
\hline & & & 2.31 & & (m) & & & 0.9 & & \\
\hline & & & & $85^{a}$ & (n) & & & & 1.0 & 1.0 \\
\hline
\end{tabular}


Table 5.3.: (continued)

\begin{tabular}{|c|c|c|c|c|c|c|c|c|c|c|}
\hline \multirow[t]{2}{*}{ Source } & \multicolumn{4}{|c|}{ Fluxes } & \multirow[b]{2}{*}{ Ref. } & \multicolumn{4}{|c|}{ SFRs } & \multirow{2}{*}{$\begin{array}{l}\text { adpt. } \\
\text { SFR }\end{array}$} \\
\hline & $\mathrm{UV}^{1}$ & $\mathrm{H}_{\alpha}^{2}$ & $\mathrm{FIR}^{3}$ & radio 4 & & UV & $\mathrm{H}_{\alpha}$ & FIR & radio & \\
\hline \multirow[t]{5}{*}{ N253 } & 16.1 & 6.06 & 100.0 & 75.4 & (e) & 2.2 & 1.4 & 9.5 & 3.0 & \\
\hline & & 6.46 & & & (d) & & 1.5 & & & \\
\hline & & 6.38 & & & (o) & & 1.5 & & & \\
\hline & & & 68.7 & & (c) & & & 6.5 & & \\
\hline & & & 70.1 & & (f) & & & 6.7 & & 4.0 \\
\hline \multirow[t]{4}{*}{ N1569 } & & 2.29 & & & (d) & & 0.15 & & & \\
\hline & & 3.14 & & & (o) & & 0.2 & & & \\
\hline & & 2.95 & & & (p) & & 0.19 & & & \\
\hline & & & 4.59 & & (q) & & & 0.12 & & 0.17 \\
\hline \multirow[t]{4}{*}{ N3628 } & & 0.32 & & & (p) & & 0.4 & & & \\
\hline & & & 3.36 & & (f) & & & 1.9 & & \\
\hline & & & 3.12 & & (r) & & & 1.8 & & \\
\hline & & & 4.17 & & (k) & & & 2.4 & & 1.6 \\
\hline \multirow[t]{3}{*}{ N4945 } & & 4.43 & 55.8 & & (k) & & 0.8 & 4.6 & & \\
\hline & & & 46.2 & & (c) & & & 3.8 & & \\
\hline & & & 37.0 & & (r) & & & 3.0 & & 3.1 \\
\hline \multirow[t]{2}{*}{ N7252 } & & & 0.30 & & (s) & & & 7.6 & & \\
\hline & & & 0.31 & & (t) & & & 7.8 & & 7.7 \\
\hline
\end{tabular}

Flux units: ${ }^{1}-10^{-25} \mathrm{erg} / \mathrm{s} / \mathrm{cm}^{2} / \mathrm{Hz} ;{ }^{2}-10^{-11} \mathrm{erg} / \mathrm{s} / \mathrm{cm}^{2} ;{ }^{3}-10^{-9} \mathrm{erg} / \mathrm{s} / \mathrm{cm}^{2} ;{ }^{4}-10^{-25} \mathrm{erg} / \mathrm{s} / \mathrm{cm}^{2} / \mathrm{Hz}$ References: (a) Buat et al. (2002), (b) Lípari et al. (2000), (c) Negishi et al. (2001), (d) Young et al. (1996), (e) Bell \& Kennicutt (2001), (f) David et al. (1992), (g) Hoopes et al. (2001), (h) Armus et al. (1990), (i) Roussel et al. (2001), (j) Rosa-González et al. (2002), (k) Lehnert \& Heckman (1996), (l) Thronson et al. (1989), (m) Viallefond et al. (1980), (n) Fabbiano et al. (1988), (o) Rownd \& Young (1999), (p) Kennicutt et al. (1994), (q) Israel (1988), (r) Rice et al. (1988), (s) Liu \& Kennicutt (1995), (t) Georgakakis et al. (2000)

${ }^{a}$ non-thermal flux, SFR conversion with formula 6.10

For each galaxy we disregarded the estimates significantly deviating from the majority of other indicators, and averaged the latter. The final values of the star formation rates we used in the following analysis are summarised in the last column of Table 5.3.

\subsubsection{Contribution of a central AGN}

As mentioned in Sec. 1 the emission of a central AGN can easily outshine the contribution of X-ray binaries. However, due to the excellent angular resolution of CHANDRA it is possible to exclude any contribution from the central AGN in nearby galaxies. In our primary sample a central AGN is present in the Circinus galaxy and NGC 4579 for which the point source associated with the nucleus of the galaxy was excluded from the luminosity 
function. Also NGC 4945 is a case where there is contribution to the X-ray emission from an AGN. However the AGN is heavily obscured and the emission below about $10 \mathrm{keV}$ of the AGN negligible (Schurch et al. 2002).

\subsubsection{Contribution of LMXBs}

Due to the absence of optical identifications of a donor star in the X-ray binaries detected by CHANDRA in other galaxies, except for LMC and SMC, there is no obvious way to discriminate the contribution of low mass X-ray binaries. On the other hand the relative contribution of LMXB sources can be estimated and, as it was mentioned above, it was one of the requirements to minimise the LMXB contribution, that determined our selection of the late-type/star-burst galaxies.

Due to the long evolution time scale of LMXBs we expect the population of LMXB sources to be roughly proportional to the stellar mass of a galaxy, whereas the population of short-living HMXBs should be defined by the very recent value of the star formation rate. Therefore the relative importance of LMXB sources should be roughly characterised by (inversely proportional to) the ratio of star formation rate to stellar mass of a galaxy. Since the determination of stellar mass, especially for a star-burst galaxy, is very difficult and uncertain we used values for the total mass of a galaxy estimated from dynamical methods and assumed that the stellar mass is roughly proportional to the total mass. To check our assumption we compare the dynamical mass with the K-band luminosity for galaxies for which, first, enough data exist to construct a growth curve in the K-band and, second, for which an extrapolation to the total $\mathrm{K}$ band flux can be made following the approach of Spinoglio et al. (1995). The number of galaxies is small, the sample consists of M 74, M 83, NGC 4736 and NGC 891, and the uncertainties associated with this approach are big, i.e. of order a factor 3. But within this uncertainty there is a correlation between the K-band luminosity and the dynamical mass estimate. However, due to the more abundant data for and higher accuracy of dynamical masses we do not use stellar mass estimates based on K-band luminosities in the following. The values of the total dynamical mass, corresponding references, and the ratios of SFR to total mass are given in Table 5.1 and Table 5.2.

The SFR to total mass ratios for late-type galaxies should be compared with that for the Milky Way, for which the population of sufficiently luminous X-ray binaries is studied rather well, chapter 3. We know that the Milky Way, having a ratio SFR/M $\mathrm{M}_{d y n} \sim 5 \cdot 10^{-13}$ $\mathrm{yr}^{-1}$, or SFR/M stellar $\sim 5 \cdot 10^{-12} \mathrm{yr}^{-1}$, is dominated by LMXB sources, HMXB sources contributing $\sim 10 \%$ to the total X-ray luminosity and $\sim 15 \%$ to the total number (above $\sim 10^{37} \mathrm{erg} / \mathrm{s}$ ) of X-ray binaries. As can be seen from Table 5.1, concerning the galaxies for which luminosity functions are available the minimal value of $\mathrm{SFR} / \mathrm{M}_{d y n} \sim 1.5 \cdot 10^{-11}$ $\mathrm{yr}^{-1}$ is achieved for M 74 and NGC 4736, which exceeds by a factor of $\sim 30$ that of the Milky Way. Therefore, even in the least favourable case of these two galaxies, we expect the HMXB sources to exceed LMXBs by a factor of $\sim 3$ at least, both in number and in luminosity.

A more detailed comparison is shown in Fig. 5.2, where we plot the expected contri- 


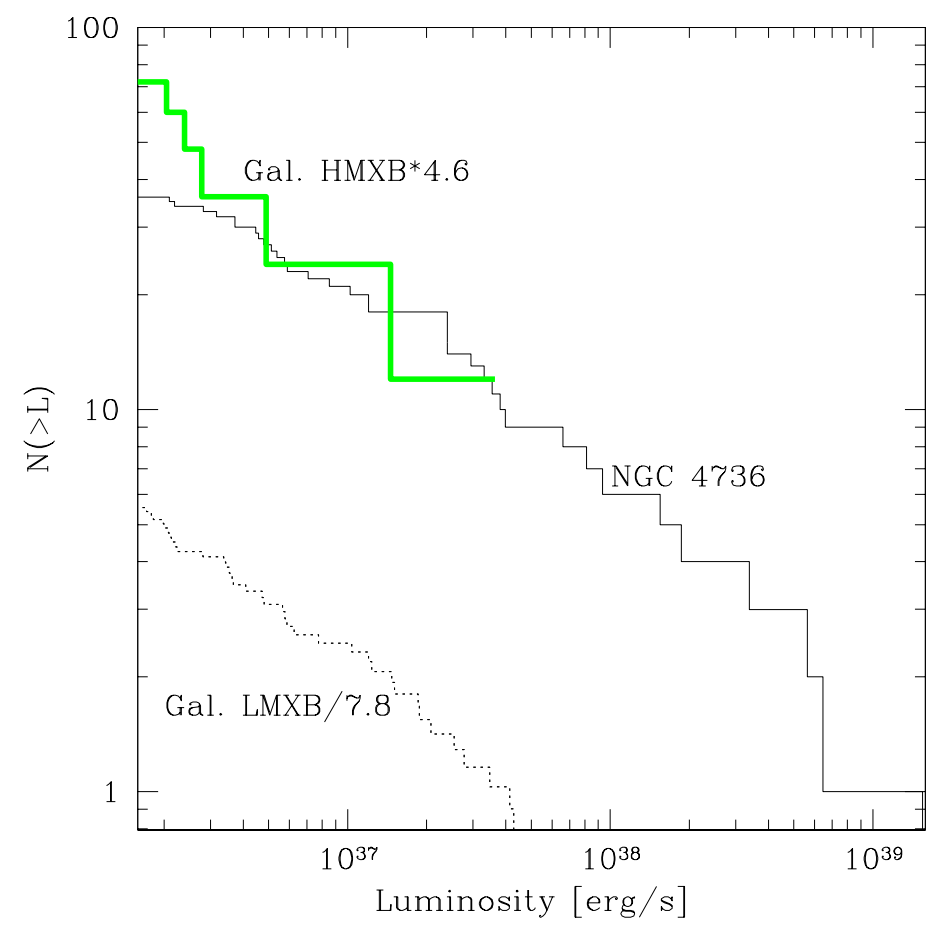

Figure 5.2.: Contributions of LMXBs and HMXBs to the observed luminosity function for NGC 4736 (thin solid histogram), having smallest SFR to total mass ratio in the primary sample. The upper thick grey histogram corresponds to the contribution of HMXBs scaled from the Milky Way HMXB luminosity function by the ratio of the SFRs. The lower dotted histogram is the Galactic LMXB luminosity function scaled by the ratio of the total masses. Total masses and SFRs are given in Table 5.1 .

butions of LMXBs and HMXBs to the observed luminosity function for NGC 4736. The luminosity function of HMXBs was obtained by scaling the Milky Way HMXB luminosity function by the ratio of SFRs of NGC 4736 to the Milky Way. The LMXB contribution was similarly estimated by scaling the Milky Way LMXB luminosity function by the ratio of the corresponding total masses. As can be seen from Fig. 5.2, the contribution of LMXB sources does not exceed $\sim 30 \%$ at the lower luminosity end of the luminosity function. If the fractions of NSs and BHs in low mass systems in late-type/star-burst galaxies are similar to that in the Milky Way, the contribution of LMXBs should be negligible at luminosities above $\sim 10^{38} \mathrm{erg} / \mathrm{sec}$, corresponding to the Eddington limit of a neutron star, to which range most of the following analysis will be restricted.

For all galaxies from Tables 5.1 and 5.2 the lowest values for SFR/M are $4 \cdot 10^{-12}$ and $9 \cdot 10^{-12}$ for IC 342 and NGC 891, respectively. This means that the contribution of LMXBs could make up a sizeable portion of their X-ray luminosity, $\sim 50 \%$ for IC 342 and $\sim 25 \%$ for NGC 891. Therefore their data points should be considered as upper limits on the integrated luminosity of HMXBs (shown in Fig. 6.5 as arrows). 


\section{High Mass X-ray Binaries as a star formation indicator}

As already mentioned, the simplest assumption about the connection of HMXBs and SFR would be that the number of X-ray sources with a high mass companion is directly proportional to the star formation rate in a galaxy. In Fig. 5.1 (right panel) we show the luminosity functions of the galaxies from our primary sample scaled to the star formation rate of the Antennae galaxies. Each luminosity function is plotted above its corresponding completeness limit. It is obvious that after rescaling the luminosity functions occupy a rather narrow band in the $\log (\mathrm{N})-\log (\mathrm{L})$ plane and seem to be consistent with each other within a factor of $\sim 2$ whereas the star formation rates differ by a factor of $\sim 40-50$. This similarity indicates that the number/luminosity function of HMXB sources might indeed be proportional to the star formation rate. This conclusion is further supported by Fig. 6.1 which shows the number of sources with a luminosity above $2 \cdot 10^{38} \mathrm{erg} / \mathrm{s}$ versus the SFR. The threshold luminosity was chosen at $2 \cdot 10^{38} \mathrm{erg} / \mathrm{s}$ to have a sufficient number of galaxies with a completeness limit equal or lower than that value and, on the other hand, to have a sufficient number of sources for each individual galaxy. In addition, as was discussed in Sec. 5.1.5, this choice of the threshold luminosity might help to minimise the contribution of LMXB sources. The errors for the number of sources were computed assuming a Poissonian distribution.

For the SFR values we assumed a 30\% uncertainty. Although the errors are rather big, the correlation of the number of sources with SFR is obvious. The slope of the correlation, determined from a least-squares fit in the form $N \propto S F R^{\alpha}$, is $\alpha=1.06 \pm 0.07$, i.e. it is consistent with unity. A fit of this correlation with a straight line $N \propto S F R$ (shown in the figure by solid line) gives:

$$
N\left(L>2 \cdot 10^{38} \mathrm{erg} / \mathrm{s}\right)=(2.9 \pm 0.23) \cdot S F R\left[\mathrm{M}_{\odot} / \mathrm{yr}\right]
$$

According to this fit we should expect less than 1 source in the Milky Way, having a SFR of $0.25 \mathrm{M}_{\odot} / \mathrm{yr}$, which is in agreement with the fact that no source above this luminosity is observed, see chapter 5.1.2.

\subsection{Universal HMXB Luminosity Function ?}

In order to check the assumption that all the individual luminosity functions have identical or similar shape with the normalisation being proportional to the SFR, we compare the luminosity function of the Antennae galaxies, having a high star formation rate $(\sim 7$ 


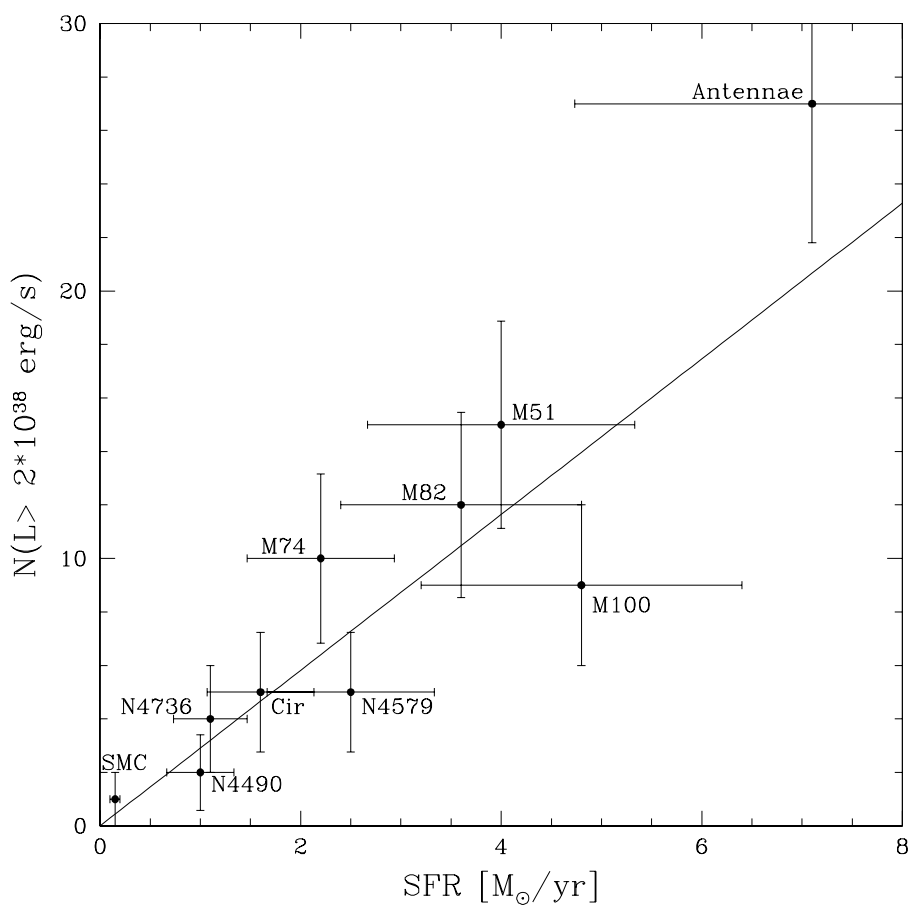

FiguRE 6.1.: Number of sources with a $2-10 \mathrm{keV}$ luminosity exceeding $2 \cdot 10^{38} \mathrm{erg} / \mathrm{s}$ versus SFR for galaxies from Table 5.1. The figure shows a clear correlation between the number of sources and the SFR. The straight line is the best fit from a Maximum-Likelihood fit, Eq. 6.1. The vertical error bars were calculated assuming a Poissonian distribution, the SFR uncertainty was assumed to be $30 \%$. For M 74 and M 100, whose completeness limit exceeds $2 \cdot 10^{38} \mathrm{erg} / \mathrm{s}$ the contribution of sources above $2 \cdot 10^{38} \mathrm{erg} / \mathrm{s}$ and below the completeness limit was estimated from the "universal" luminosity function, Eq. 6.3.

$\mathrm{M}_{\odot} / \mathrm{yr}$ ), with the collective luminosity function of galaxies with medium SFRs (in the range of $\left.\sim 1.0-3.5 \mathrm{M}_{\odot} / \mathrm{yr}\right)$. For the later we summed the luminosity functions of M 82, NGC 4579, NGC 4736 and Circinus, having a combined SFR of $\sim 8.8 \mathrm{M}_{\odot} / \mathrm{yr}$. The two luminosity functions (shown in Fig. 6.2) agree very well at $L_{X}<10^{39} \mathrm{erg} / \mathrm{s}$ with possible differences at higher luminosities. In a strict statistical sense, a Kolmogorov-Smirnov test gives a $15 \%$ probability that the luminosity functions are derived from the same distribution, thus, neither confirming convincingly, nor rejecting the null hypothesis. However, it should be emphasised, that whereas the shape of a single slope power law luminosity function is not affected at all by the uncertainty in the distance, more complicated forms of a luminosity function, e.g. a power law with cut-off, would be sensitive to errors in the distance determination. The effect might be even stronger for the combined luminosity functions of several galaxies, located at different distances and each having different errors in the distance estimate. In the case of a power law with high luminosity cut-off, the effect would be strongest at the high luminosity end and will effectively dilute the cut-off, as probably is observed. Therefore, we can presently not draw a definitive conclusion about the existence of a universal luminosity function of HMXBs, from which all luminosity functions of the individual galaxies are strictly derived. For instance, subtle effects similar to the effect of flattening of the luminosity function with increase of SFR suggested by Kilgard et al. (2001); Ghosh \& White (2001); Ptak et al. (2001) can not be excluded based on the presently available sample of galaxies and sensitivities achieved. We can conclude, however, that there is no evidence for strong non-linear dependences of the luminosity 


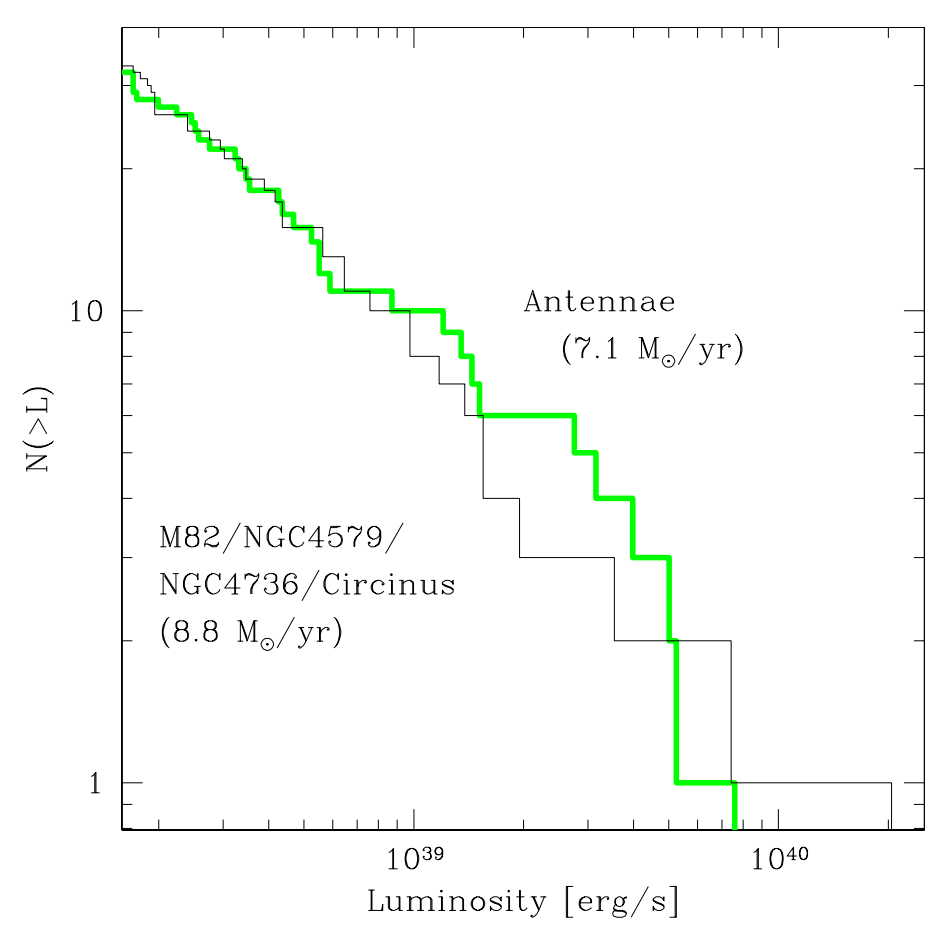

FIGURE 6.2.: Comparison of the combined luminosity function of $\mathrm{M}$ 82, NGC 4579, NGC 4736 and Circinus, having SFRs in the range 1$3.5 \mathrm{M}_{\odot} / \mathrm{yr}$ with the Antennae luminosity function $\left(7.1 \mathrm{M}_{\odot} / \mathrm{yr}\right)$. A Kolmogorov-Smirnov test gives a probability of $15 \%$ that the two luminosity functions are derived from the same distribution. See discussion in the text regarding the effect of the errors in the distance measurements on the shape of the combined luminosity function.

function on the SFR.

As the next step we compare the luminosity functions of actively star forming galaxies with that of low SFR galaxies. Unfortunately, the X-ray binary population of low SFR galaxies is usually dominated by LMXB systems. One of the cases in which the luminosity function of HMXB sources can be reliably obtained is the Milky Way galaxy, for which all sufficiently bright X-ray binaries are optically identified. Another case is the Small Magellanic Cloud, which has a SFR value similar to our Galaxy, but is $\sim 300-500$ less massive and, correspondingly, has very few, if any, LMXB sources (Yokogawa et al. 2000). Moreover, the SMC is close enough to have optical identifications of HMXBs which makes a distinction like in the Milky Way possible. In order to do the comparison, we combined the luminosity functions of all actively star forming galaxies from our sample with a completeness limit lower than $2 \cdot 10^{38} \mathrm{erg} / \mathrm{sec}-\mathrm{M} 82$, Antennae, NGC 4579, NGC 4736 and Circinus. These galaxies have a total SFR of $\sim 16 \mathrm{M}_{\odot} / \mathrm{yr}$, which exceeds the Milky Way SFR $\left(\sim 0.25 \mathrm{M}_{\odot} / \mathrm{yr}\right)$ by a factor of $\sim 65$. Fig. 6.3 shows the combined luminosity function of the above mentioned star forming galaxies and the luminosity functions of Galactic and SMC HMXBs scaled according to the ratios of SFRs. Shown in Fig. 6.3 by a solid line is the fit to the luminosity function of the high SFR galaxies only (see below), extrapolated to lower luminosities. It is obvious that the luminosity functions of Galactic and SMC HMXBs agree surprisingly well with an extrapolation of the combined luminosity function of the star-burst galaxies.

Thus we demonstrated that the presently available data are consistent with the assump- 

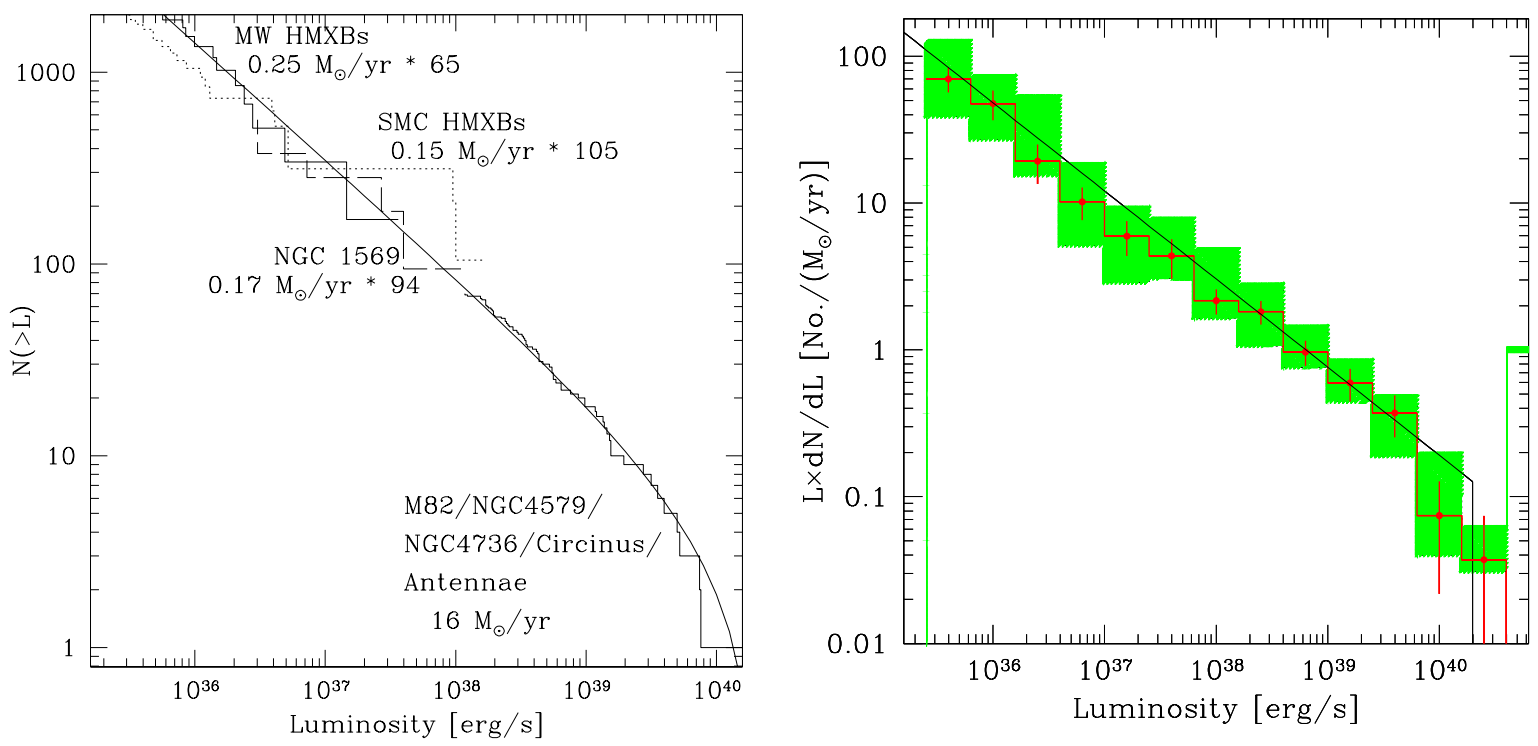

Figure 6.3.: Left: Combined luminosity function of compact X-ray sources in the star-burst galaxies M82, NGC 4038/9, NGC 4579, NGC 4736 and Circinus with a total SFR of $16 \mathrm{M}_{\odot} / \mathrm{yr}$ (histogram above $2 \cdot 10^{38} \mathrm{erg} / \mathrm{s}$ ) and the luminosity functions of NGC 1569 and HMXBs in the Milky Way and Small Magellanic Clouds (three histograms below $2 \cdot 10^{38} \mathrm{erg} / \mathrm{s}$ ). The thin solid line is the best fit to the combined luminosity function of the star-burst galaxies only, given by Eq. 6.3. Right: Differential luminosity function obtained by combining the data for all galaxies from the primary sample, except for NGC 3256 (see text). The straight line is the best fit to the luminosity function of star forming galaxies given by Eq. 6.2. - the same as in the left hand panel. Note, that due to different construction algorithms, the luminosity functions shown in the left and right panels are based on different but overlapping samples of galaxies (see discussion in the text). The grey area is the $90 \%$ confidence level interval we obtained from a Monte-Carlo simulation taking into account uncertainties in the SFR and distances. For details see discussion in the text.

tion that the approximate shape and normalisation of the luminosity function for HMXBs in a galaxy with a known star formation rate can be derived from a "universal" luminosity function whose shape is fixed and whose normalisation is proportional to star formation rate. Due to a number of uncertainties involved, the accuracy of this approximation is difficult to assess. Based on our sample of galaxies we can conclude that it might be accurate within $\sim 50 \%$.

In order to obtain the universal luminosity function of HMXBs we fit the combined luminosity function of M 82, Antennae, NGC 4579, NGC 4736 and Circinus using a MaximumLikelihood method with a power law with a cut-off at $L_{c}=2.1 \cdot 10^{40} \mathrm{erg} / \mathrm{s}$ and normalise the result to the combined SFR of the galaxies. The best fit luminosity function (solid line in Fig.6.3) in the differential form is given by:

$$
\frac{d N}{d L_{38}}=\left(3.3_{-0.8}^{+1.1}\right) \cdot S F R \cdot L_{38}^{-1.61 \pm 0.12} \text { for } L<L_{c}
$$


where $L_{38}=L / 10^{38} \mathrm{erg} / \mathrm{sec}$ and SFR is measured in units of $\mathrm{M}_{\odot} / \mathrm{yr}$. The errors are $1 \sigma$ estimates for one parameter of interest. The rather large errors for normalisation are due to the correlation between slope and normalisation of the luminosity function, with a higher value of normalisation corresponding to a steeper slope. The cumulative form of the luminosity function, corresponding to the best values of the slope and normalisation is:

$$
N(>L)=5.4 \cdot S F R \cdot\left(L_{38}^{-0.61}-210^{-0.61}\right)
$$

According to a Kolmogorov-Smirnov test the data are consistent with the best fit model at a confidence level of $90 \%$.

As an additional test we checked all individual luminosity functions against our best fit using a Kolmogorov-Smirnov test. Taking into account the respective completeness limits, the shapes of all individual luminosity functions are compatible with the assumption of a common 'origin'. In Fig. 6.4 we show the individual luminosity functions along with the universal luminosity function given by Eq.(6.2) with the normalisation determined according to the corresponding star formation rates derived from the conventional SFR indicators (Table 5.1).

Finally, we construct the differential luminosity function combining the data for all galaxies from the primary sample, except for NGC 3256 (having somewhat uncertain completeness limit). To do so we bin all the sources above the corresponding completeness limits in logarithmically spaced bins and normalise the result by the combined SFR of all galaxies contributing to a given bin. Such a method has the advantage of using all the available data. A disadvantage is that due to significantly different luminosity ranges of the individual luminosity functions (especially SMC and Milky Way on one side and star forming galaxies on the other) uncertainties in the conventional SFR estimates may lead to the appearance of artificial features in the combined luminosity function. With that in mind, we plot the differential luminosity function in the right panel of Fig. 6.3 along with the best fit power law from Eq.(6.2).

In order to investigate the influence of systematic uncertainties in SFR and distance we performed a Monte-Carlo simulation taking into account these two effects. The grey area in the right panel of Fig. 6.3 shows the $90 \%$ confidence interval obtained from this simulation. In the simulation we randomly varied the distances of galaxies, assuming the errors on the distance to be distributed according to a Gaussian with a mean of 0 and a width of $20 \%$ of the distance of a galaxy which corresponds to an uncertainty in luminosity of $\sim 40 \%$. Correspondingly the SFR, affected in the same way as the X-ray luminosity by uncertainties in the distance, was changed. Additionally the SFR was randomly varied also assuming a Gaussian error distribution with a mean of 0 and a width of $30 \%$ of the SFR, as assumed for Fig. 6.1. For the Milky Way we varied in each Monte-Carlo run the distance to each HMXB independently with a Gaussian with a mean of 0 and a width of $20 \%$ of the distance.

Noteworthy is the fact that the luminosity function is sufficiently close to a single slope power law in a broad luminosity range covering more than five orders of magnitude. If the absence of significant features is confirmed this allows to constrain the relative abundance 


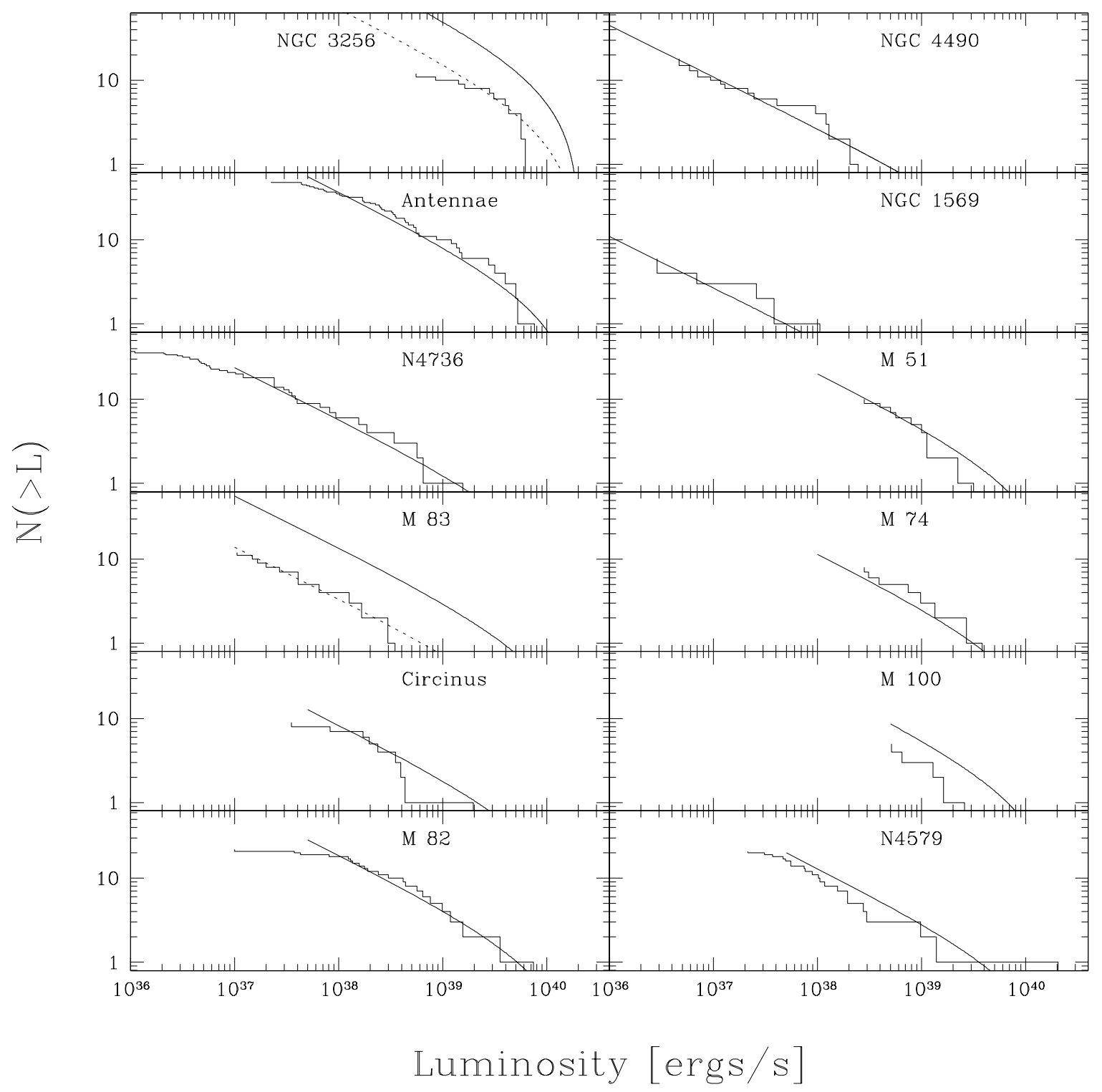

Figure 6.4.: Comparison of the "universal" luminosity function defined by Eq. 6.2 (thin solid lines) with individual luminosity functions of compact X-ray sources in the galaxies from Table 5.1 (histograms). The normalisation of the "universal" luminosity function in each panel was calculated using corresponding SFR values from Table 5.1. For M83 the luminosity function of the compact sources in the nuclear region only is plotted, whereas the normalisation of the "universal" luminosity function was computed using the overall SFR for the galaxy. Therefore the thin line should be considered as an upper limit. The dotted lines are fits to the normalisation of the observed luminosity functions in the cases where completeness or coverage do not represent the same area as the SFR measurements. 
of NS and BH binaries and/or the properties of accreting compact objects at supercritical accretion rates (see discussion in Sec. 6.7.1).

However, it should be emphasised that there is hardly any overlap in the luminosity functions for low and high SFR galaxies, as is obvious from Fig. 5.1 and 6.3. It happens that this gap is around the Eddington luminosity of a NS, $L_{E d d, N S}$, which should be a dividing line between NS and BH binaries. From simple assumptions it would be expected that the luminosity functions below $L_{E d d, N S}$ are dominated by NS whereas above $L_{E d d, N S}$ $\mathrm{BH}$ binaries should dominate. This would imply a break in the luminosity function around $L_{E d d, N S}$ because of different abundances of NSs and BHs. Due to the uncertainties in SFR measurements it is possible that a break, that would theoretically be expected around $L_{E d d, N S}$, could be hidden by this gap. Even upper limits (not more than twice) are of importance and could give some additional information about the relative strength of the two populations of accreting binaries (see discussion in Sec. 6.7). Observations of star forming galaxies with sufficient sensitivity, i.e. with a completeness limit well below $10^{38}$ $\mathrm{erg} / \mathrm{s}$ will be able to resolve this question.

\subsection{High Luminosity cut-off}

The combined luminosity function shown in the left panel of Fig. 6.3 indicates a possible presence of a cut-off at $L_{c} \sim 2 \cdot 10^{40} \mathrm{erg} / \mathrm{s}$. From a statistical point of view, when analysing the combined luminosity function of the high SFR galaxies only, the significance of the cut-off is not very high, with a single slope power law with slope 0.74 for the cumulative luminosity function also giving an acceptable fit, although with a somewhat lower probability of $54 \%$ according to a Kolmogorov-Smirnov test. However, an independent strong evidence for the existence of a cut-off around $\sim$ few $\cdot 10^{40} \mathrm{erg} / \mathrm{s}$ is provided by the $L_{X}-\mathrm{SFR}$ relation as discussed in the next sub-chapters.

The existence of such a cut-off, if it is real and if it is a universal feature of the HMXB luminosity function, can have significant implications to our understanding of the so-called ultra-luminous X-ray sources. Assuming that these very luminous objects are intermediate mass BHs accreting at the Eddington limit, the value of the cut-off gives an upper limit on the mass of the black hole, $\sim 100 \mathrm{M}_{\odot}$. These apparently super-Eddington luminosities can also be the result of other effects, like a strong magnetic field in NSs which may allow radiation to escape without interacting with the accreting material (Basko \& Sunyaev 1976), emission from a supercritical accretion disk (Shakura \& Sunyaev 1973; Paczynsky \& Wiita 1980), beamed emission (King et al. 2001), or the emission of a jet as suggested by Körding et al. (2002). Moreover, in BHs in high state radiation is coming from the quasiflat accretion disk where electron scattering gives the main contribution to the opacity. It is easy to show that the radiation flux perpendicular to the plane of the disk exceeds the average value by up to 3 times (Shakura \& Sunyaev 1973). Also the Eddington luminosity is dependent on chemical abundance which allows a twice higher luminosity for accretion of helium. These last two effects alone can provide a factor of 6 above the canonical Eddington luminosity. 
It should be mentioned that, based on the combined luminosity function only, we can not exclude the possibility that the cut-off is primarily due to the Antennae galaxies which contributes about half of the sources above $10^{39} \mathrm{erg} / \mathrm{s}$ and shows a prominent cut-off in its luminosity function. On the other hand, further indication for a cut-off is provided by the luminosity function of NGC 3256. Conventional star formation indicators give a value of SFR of $\sim 45 \mathrm{M}_{\odot} / \mathrm{yr}$, however its luminosity function also shows a cut-off at $\sim 10^{40} \mathrm{erg} / \mathrm{s}$. Unfortunately, due to the large distance $(35 \mathrm{Mpc})$ and a comparatively short exposure time of the CHANDRA observation, $\sim 28 \mathrm{ks}$, the luminosity function of NGC 3256 becomes incomplete at luminosities shortly below the brightest source and therefore does not allow for a detailed investigation.

\subsection{Total X-ray luminosity as SFR indicator}

CHANDRA and future X-ray missions with angular resolution of the order of $\sim 1^{\prime \prime}$ would be able to spatially resolve X-ray binaries only in nearby galaxies $(d<\sim 50-100 \mathrm{Mpc})$. For more distant galaxies only the total luminosity of a galaxy due to HMXBs can be used for X-ray diagnostics of star formation.

Fig. 6.5 shows the total luminosity of X-ray binaries (above $10^{36} \mathrm{erg} / \mathrm{s}$ ) plotted versus SFR. The galaxies from the primary sample (listed in Table 5.1) are shown by filled circles. The galaxies, for which only total luminosity is available (Table 5.2) are shown as filled triangles. The luminosities of the galaxies from the primary sample were calculated by summing the luminosities of individual sources down to the completeness limit of the corresponding luminosity function. The contribution of the sources below the completeness limit was approximately accounted for by integrating a power law distribution with slope 1.6 and normalisation obtained from the fit to the observed luminosity function. Note, that due to the shallow slope of the luminosity function the total luminosity depends only weakly on the lower integration limit.

As an additional data point we take luminosity and SFR for the Large Magellanic Cloud. The SFR is similar to the Milky Way SFR (Holtzman et al. 1999). Since no luminosity function is presently available for LMC we estimated its integrated X-ray luminosity as a sum of the time averaged luminosities of the three brightest HMXB sources (LMC X-1, $\mathrm{X}-3, \mathrm{X}-4)$ as measured by ASM, see Table $3.2 .3, L_{2-10 \mathrm{keV}} \approx 3.4 \cdot 10^{38} \mathrm{erg} / \mathrm{s}$. Contribution of the weaker sources should not change this estimate significantly, since the luminosity of the next brightest source is by a factor of $\sim 30-50$ smaller (Sunyaev et al. 1990).

\subsection{Theoretical $L_{X}-\mathrm{SFR}$ relation}

At first glance, the relation between collective luminosity of HMXBs and SFR can be easily derived integrating Eq. (6.2) for the SFR dependent luminosity function. Therefore, as the population of HMXB sources in a galaxy is directly proportional to SFR, one might expect that the X-ray luminosity of galaxies due to $\mathrm{HMXB}, L_{X}$, should be linearly proportional 
to SFR. However this problem contains some subtleties related to the statistical properties of the power law luminosity distribution of discrete sources which appear not to have been recognised previously (at least in astrophysical context). The difference between the most probable value of the total luminosity of HMXB sources in a galaxy (the mode of the distribution) and the ensemble average value (expectation mean, obtained by integrating Eq. (6.2)) results in the non-linear $L_{X}$-SFR dependence in the low SFR regime. This effect will be discussed more detailed and rigorously in chapter 6.8 , here we give only a short introduction. A somewhat similar problem was considered by Kalogera et al. (2001) in the context of pulsar counts and the faint end of the pulsar luminosity function.

For illustration only, let us consider a population of discrete sources with a Gaussian luminosity function. As is well known, in this case the sum of their luminosities - the integrated luminosity of the parent galaxy, also obeys a Gaussian distribution for which the mean luminosity and dispersion can be computed straightforwardly. An essential property of this simple case is that for an ensemble of galaxies, each having a population of such sources, the most probable value of the integrated luminosity of an arbitrarily chosen galaxy (the mode of the distribution) equals to the mean luminosity (averaged over the ensemble of galaxies). The situation might be different in the case of a population of discrete sources with a power law (or similarly skewed) luminosity function. In this case an ensemble of galaxies would have a non-Gaussian probability distribution of the integrated luminosity. Due to skewness of the probability distribution in this case, the most probable value of the integrated luminosity of an arbitrarily chosen galaxy does not necessarily coincide with the mean value (the ensemble average). The effect is caused by the fact that depending on the slope of the luminosity function and its normalisation the integrated luminosity of the galaxy might be defined by a small number of brightest sources even when the total number of sources is large. Of course, in the limit of large number of sources in the high luminosity end of the luminosity function the distribution becomes asymptotically close to Gaussian and, correspondingly, the difference between the most probable value and the ensemble average vanishes. In this limit the relation between the integrated luminosity of HMXBs and SFR can be derived straightforwardly integrating Eq.(6.2) for $L_{c}=2.1 \cdot 10^{40}$ $\mathrm{erg} / \mathrm{s}$

$$
L_{X}=6.7 \cdot 10^{39} \cdot S F R\left[\mathrm{M}_{\odot} / \mathrm{yr}\right] \mathrm{erg} / \mathrm{s}
$$

It should be emphasised that the ensemble average integrated luminosity (i.e. averaged over many galaxies with similar SFR) is always described by the above equation, independent of the number of sources and shape of the luminosity function. This equality is maintained due to the outlier galaxies, whose luminosity exceeds significantly both the most probable and average values. These outlier galaxies will result in enhanced and asymmetric dispersion in the low SFR-regime.

The following simple consideration leads to an approximate analytical expression for the most probable value of the integrated luminosity. Assuming a power law luminosity function $d N / d L=A \cdot S F R \cdot L^{-\alpha}$ with $1<\alpha<2$, one might expect, that the brightest source would most likely have a luminosity $L_{\max }$ close to the value $\sim L_{1}$ such that $N(>$ 
$\left.L_{1}\right) \sim 1$, i.e.

$$
L_{1} \propto S F R^{\frac{1}{\alpha-1}}
$$

In the presence of a cut-off $L_{c}$ in the luminosity function, the luminosity of the brightest source, of course, can not exceed the cut-off luminosity: $L_{\max }=\min \left(L_{1}, L_{c}\right)$. The most probable value of the total luminosity can be computed integrating the luminosity function from $L_{\min }$ to $L_{\max }=\min \left(L_{1}, L_{c}\right)$ :

$$
L_{\text {total }}=\int_{L_{\text {min }}}^{\min \left(L_{1}, L_{c}\right)} \frac{d N}{d L} L d L
$$

which leads to

$$
L_{\text {total }} \approx \frac{A \cdot S F R}{2-\alpha} \cdot \min \left(L_{1}, L_{c}\right)^{2-\alpha}
$$

for $1<\alpha<2$ and $L_{1}, L_{c}>>L_{\min }$.

Obviously there are two limiting cases of the $L_{X}-$ SFR dependence of the total luminosity on SFR, depending on the relation between $L_{c}$ and $L_{1}$, i.e. on the expected number of sources in the high end of the luminosity function, near its cut-off. In the limit of low SFR (small number of sources) $L_{1}<L_{c}$ and the luminosity of the brightest source would increase with SFR: $L_{\max } \sim L_{1} \propto S F R^{\frac{1}{\alpha-1}}$. Therefore the $L_{X}-$ SFR dependence might be strongly non-linear:

$$
L_{\text {total }} \propto S F R^{\frac{1}{\alpha-1}}
$$

e.g. for $\alpha=1.5$ the relation is quadratic $L_{\text {total }} \propto S F R^{2}$. For sufficiently large values of SFR $L_{1}>L_{c}$, i.e. $N\left(>L_{c}\right)>1$ implying a large number of sources in the high luminosity end of the luminosity function and, correspondingly, Gaussian probability distribution of the integrated luminosity. In this case $L_{\max } \sim L_{c}=$ const and does not depend on SFR anymore and the dependence is linear, in accord with Eq.(6.4).

Importantly, the entire existence of the linear regime in the $L_{X}-\mathrm{SFR}$ relation is a direct consequence of the existence of a cut-off in the luminosity function. For a sufficiently flat luminosity function, $1<\alpha<2$, the collective luminosity of the sources grows faster than linear because brighter and brighter sources define the total luminosity as the star formation rate increases. Only in the presence of the maximum possible luminosity of the sources, $L_{c}$ (for instance Eddington limit for NSs) the regime can be reached, when $N\left(>L_{c}\right)$ becomes larger than unity and subsequent increase of the star formation rate results in the linear growth of the total luminosity. The latter, linear, regime of the $L_{X}-\mathrm{SFR}$ relation was studied independently by Ranalli et al. (2003) based on ASCA and BeppoSAX data. Note that their equation (12) agrees with our Eq.(6.4) within $15 \%$.

The position of the break in the $L_{X}$-SFR relation depends on the slope of the luminosity function and the value of the cut-off luminosity:

$$
\mathrm{SFR}_{\text {break }} \propto L_{c}^{\alpha-1}
$$




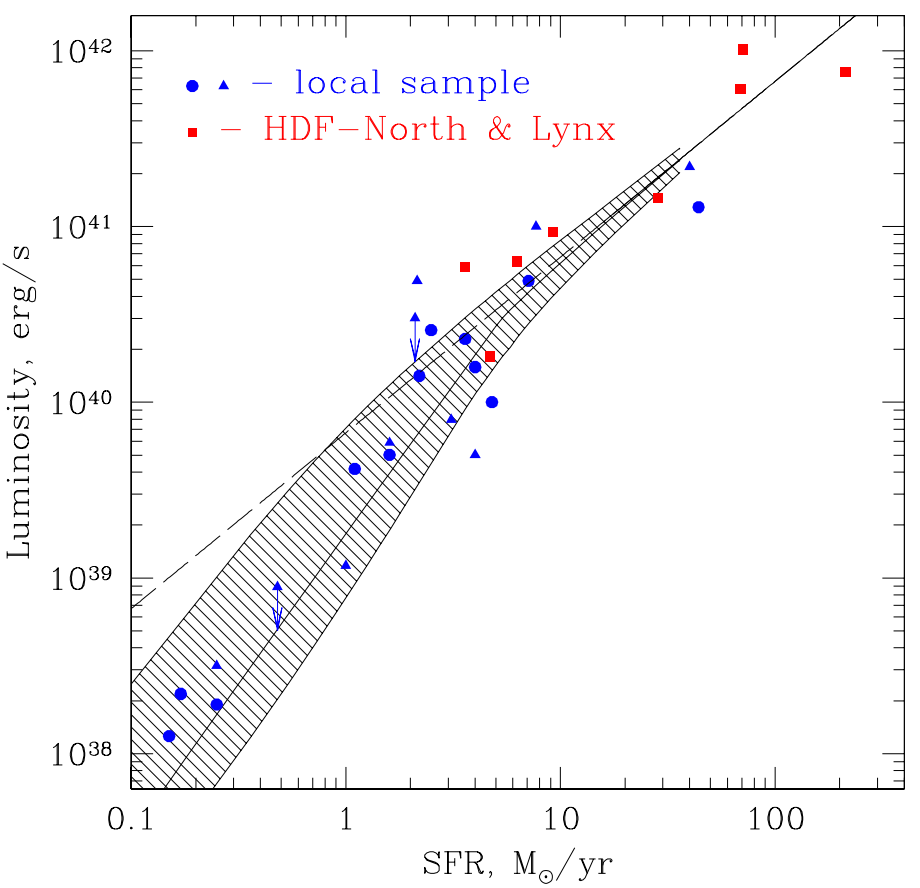

FIGURE 6.5.: The $L_{X}-$ SFR relation. The filled circles and triangles are nearby galaxies from Table 5.1 (primary sample) and Table 5.2 (secondary sample), the open circles are distant star forming galaxies from the HDF North and Lynx field. The arrows are the upper limits for the X-ray luminosity due to HMXBs for IC 342 and NGC 891. The thick solid line shows the expected relation between SFR and the most probable value of the total luminosity computed for the best fit parameters of the HMXB luminosity function. Note, that in the low SFR regime the probability to find a galaxy below the solid curve is $\sim$ $10-15 \%$. The shaded area shows the $68 \%$ confidence region including both intrinsic variance of the $L_{X}-\mathrm{SFR}$ relation and uncertainty of the best fit parameters of the HMXB luminosity function (Eq.(6.2)). The dashed line shows the linear $L_{X}$-SFR relation given by Eq.(6.4).

Combined with the slope of the $L_{X}$-SFR relation in the low SFR regime (Eq.(6.8)) and the normalisation of the linear dependence in the high SFR limit this opens a possibility to constrain the parameters of the luminosity function studying the $L_{X}$-SFR relation alone, without actually constructing luminosity functions, e.g. in distant unresolved galaxies.

\section{5. $L_{X}-\mathrm{SFR}$ relation: comparison with the data}

The solid line in Fig.6.5 shows the result of the exact calculation of the $L_{X}-\mathrm{SFR}$ relation from chapter 6.8. The relation was computed for the best fit parameters of the HMXB luminosity function determined from the analysis of five mostly well studied galaxies from the primary sample (chapter 6.1 and Eq.(6.2)). Note, that due to the skewness of the probability distribution for $L_{\text {total }}$ in the non-linear, low SFR regime the theoretical probability to find a galaxy below the most probable value (the solid curve in Fig.6.5) is $\approx 12-16 \%$ at $\mathrm{SFR}=0.2-1.5 \mathrm{M}_{\odot} / \mathrm{yr}$ and increases to $\approx 30 \%$ at SFR $=4-5 \mathrm{M}_{\odot} / \mathrm{yr}$, near the break of the $L_{X}-\mathrm{SFR}$ relation. In the linear regime $\left(\mathrm{SFR}>10 \mathrm{M}_{\odot} / \mathrm{yr}\right)$ it asymptotically approaches 
$\sim 50 \%$, as expected. The shaded area around the solid curve corresponds to the $68 \%$ confidence level including both intrinsic variance of the $L_{X}-\mathrm{SFR}$ relation and uncertainty of the best fit parameters of the HMXB luminosity function (Eq.(6.2)).

Fig.6.5 demonstrates sufficiently good agreement between the data and the theoretical $L_{X}$-SFR relation. Importantly, the predicted relation agrees with the data both in the high and low SFR regime, thus showing that the data including the high redshift galaxies from Hubble Deep Field North (see the following sub-chapter), are consistent with the HMXB luminosity function parameters, derived from significantly fewer galaxies than plotted in Fig.6.5.

The existence of the linear part at SFR $>5-10 \mathrm{M}_{\odot} /$ yr gives an independent confirmation of the reality of the cut-off in the luminosity function of HMXBs (cf. Sec. 6.2). The position of the break and normalisation of the linear part in the $L_{X}-\mathrm{SFR}$ relation confirms that the maximum luminosity of the HMXB sources (cut-off in the HMXB luminosity function) is of the order of $L_{c} \sim 10^{40}-10^{41} \mathrm{erg} / \mathrm{s}$ (see chapter 6.8 for more details). Despite the number of theoretical ideas being discussed, the exact reason for the cut-off in the HMXB luminosity function is not clear and significant variations of $L_{c}$ among galaxies, related or not to the galactic parameters, such as metalicity or star formation rate can not be excluded a priori. However, significant variations in $L_{c}$ from galaxy to galaxy would result in large dispersion in the break position and in the linear part of the $L_{X}-\mathrm{SFR}$ relation. As such large dispersion is not observed, one might conclude that there is no large variation of the cut-off luminosity between galaxies and, in particular, there is no strong dependence of the cut-off luminosity on SFR.

\subsection{Hubble Deep Field North}

In order to check whether the correlation, which is clearly seen from Fig. 6.5 for nearby galaxies, holds for more distant galaxies as well we used the data of the CHANDRA observation of the Hubble Deep Field North (Brandt et al. 2001). We cross-correlated the list of the X-ray sources detected by CHANDRA with the catalogue of radio sources detected by VLA at $1.4 \mathrm{GHz}$ (Richards 2000). Using optical identifications of Richards et al. (1998) and redshifts from Cohen et al. (2000) we compiled a list of galaxies detected by CHANDRA and classified as spiral or irregular/merger galaxies by Richards et al. (1998) and not known to show AGN activity. The K-correction for radio luminosity was done assuming a power law spectrum and using the radio spectral indices from Richards (2000). The X-ray luminosity was K-corrected and transformed to the 2-10 keV energy range using photon indices from Brandt et al. (2001). The final list of galaxies selected is given in Table 6.1. An additional data point, X-ray flux and redshift, is taken from the observation of the Lynx Field by Stern et al. (2002). The radio flux is obtained from a cross-correlation of the X-ray positions with Oort (1987).

The star formation rates were calculated assuming that the non-thermal synchrotron emission due to electrons accelerated in supernovae dominates the observed $1.4 \mathrm{GHz}$ lumi- 
TABle 6.1.: Sample galaxies from the Hubble Deep Field North and Lynx Field.

\begin{tabular}{|l|c|c|c|c|c|}
\hline Source & redshift & $\begin{array}{c}F_{1.4 \mathrm{GHz}} \\
{[\mu \mathrm{Jy}]}\end{array}$ & $\begin{array}{c}\mathrm{SFR} \\
{\left[\mathrm{M}_{\odot} / \mathrm{yr}\right]}\end{array}$ & $\begin{array}{c}S_{0.5-8 \mathrm{keV}} \\
{\left[10^{-15} \mathrm{erg} / \mathrm{s} / \mathrm{cm}^{2}\right]}\end{array}$ & $\begin{array}{c}L_{X} \\
{\left[10^{40} \mathrm{erg} / \mathrm{s}\right]}\end{array}$ \\
\hline $123634.5+621213$ & 0.458 & 233 & 28 & 0.43 & 14.4 \\
$123634.5+621241$ & 1.219 & 230 & 213 & 0.3 & 75.9 \\
$123649.7+621313$ & 0.475 & 49 & 8 & 0.15 & 2.5 \\
$123651.1+621030$ & 0.410 & 95 & 9 & 0.3 & 9.3 \\
$123653.4+621139$ & 1.275 & 66 & 69 & 0.22 & 60.6 \\
$123708.3+621055$ & 0.423 & 45 & 4 & 0.18 & 5.9 \\
$123716.3+621512$ & 0.232 & 187 & 5 & 0.18 & 1.8 \\
\hline $084857.7+445608$ & 0.622 & 320 & 71 & 1.46 & 102 \\
\hline
\end{tabular}

For two galaxies, $123634.5+621213$ and $123651.1+621030$, there exist stellar mass estimates obtained with the method of Brinchmann \& Ellis (2000) of $4.2 \cdot 10^{11} \mathrm{M}_{\odot}$ and $7 \cdot 10^{10} \mathrm{M}_{\odot}$ respectively, which show that the galaxies are dominated by $\operatorname{HMXBs}(\mathrm{J}$. Brinchmann, private communication).

nosity and using the following relation from Condon (1992):

$$
S F R_{\text {radio }}=1.9 \cdot 10^{-29} \cdot \nu_{\mathrm{GH} z}^{\alpha} \cdot L_{\nu}[\mathrm{erg} / \mathrm{s} / \mathrm{Hz}],
$$

where $\alpha$ is the slope of the non-thermal radio emission.

The galaxies from HDF North and Lynx are shown in Fig.6.5 by open circles. A sufficiently good agreement with the theoretical $L_{X}-\mathrm{SFR}$ relation is obvious.

\subsection{Discussion}

\subsubsection{Neutron stars, stellar mass black holes and intermediate mass black holes}

Two well known and one possible types of accreting objects should contribute to the X-ray luminosity function of sources in star forming galaxies:

1. neutron stars $\left(\mathrm{M} \sim 1.4 \mathrm{M}_{\odot}\right)$,

2. stellar mass black holes $\left(3 \leq M / M_{\odot} \leq 20\right)$ born due to collapse of high mass stars, and

3. intermediate mass $\left(50<M / M_{\odot}<10^{5}\right)$ black holes of unknown origin.

Each class of accreting objects is expected to have a maximum possible luminosity, close or exceeding by a factor of several the corresponding Eddington luminosity. In a general case we should expect that each of these three types of accreting objects should have its own luminosity function depending on the mass distribution inside each class (more narrow for NSs, more broad for BHs and probably very broad for intermediate mass BHs), properties 
of the binary and mass loss type and rate from the normal star. Therefore, the combined luminosity function of a galaxy, containing all three types of objects should have several breaks or steps (see Fig. 6.6) which are not present in Fig. 6.3. Such breaks should be connected with the fact that, for example, below the Eddington limit for a NS (or at somewhat higher luminosity) more abundant NS X-ray binaries might dominate in the number of objects, whereas at higher luminosities only black holes should contribute due to their higher masses and broader mass distribution. Until now CHANDRA data did not show any evidence for a break in the luminosity function expected in the vicinity or above of Eddington luminosity for NS mass. However, such a break must exist, the only question is how pronounced and broad it is.

It is believed that stars with masses higher than 60-100 $M_{\odot}$ are unstable. Therefore there should be an upper limit on the mass of BHs born as a result of stellar collapse. Until now the most massive known stellar mass BH in our Galaxy, GRS 1915+105, has a mass of $\sim 15 \mathrm{M}_{\odot}$ (Greiner et al. 2001). It is natural that the Eddington luminosity of these objects, amplified several times by angular distribution of radiation and chemical abundance effects, should result in the maximum luminosity of X-ray sources of this type. It is important to mention that 3 years of RXTE/ASM observations revealed from time to time super-Eddington luminosities of some Galactic X-ray binaries on the level of 3-12 $L_{E d d, N S}$, see Table 3.2.3.

The hypothetical intermediate mass BHs, probably reaching masses of $\sim 10^{2-5} M_{\odot}$, might be associated with extremely high star formation rates (BHs merging in dense stellar cluster etc.) and are expected to be significantly less frequent than $\sim$ stellar mass BHs. Therefore the transition from the $\sim$ stellar mass BH HMXB luminosity function to intermediate mass $\mathrm{BHs}$ should be visible in the cumulative luminosity function. Merging BHs are one possible way of rapid growth of super-massive BHs that exist in practically all galaxies. To accrete efficiently intermediate mass BHs should form close binary systems with normal stars or be in dense molecular clouds.

If the cut-off in the luminosity function, observed at $\sim$ few $10^{40} \mathrm{erg} / \mathrm{s}$ corresponds to the maximum possible luminosity of $\sim$ stellar mass $\mathrm{BHs}$ and if at $L>L_{c}$ the population of hypothetical intermediate mass BHs emerges, it should lead to a drastic change in the slope of the $L_{X}$-SFR relation at extreme values of SFR. Therefore, observations of distant star forming galaxies with very high SFR might be one of the best and easiest ways to probe the population of intermediate mass black holes.

\section{Three component luminosity function}

In Fig. 6.6 we present the result of a simple picture of what type of universal luminosity function a very simple model of HMXB population synthesis could produce. This picture is obviously oversimplified but we present it here to show that the simple picture cannot reproduce the smooth luminosity function we get from CHANDRA observations of star forming galaxies.

The initial set-up includes parameterisation of the mass distributions of NSs and BHs, the distribution of mass transfer rates in binary systems, and a prescription for the conversion 


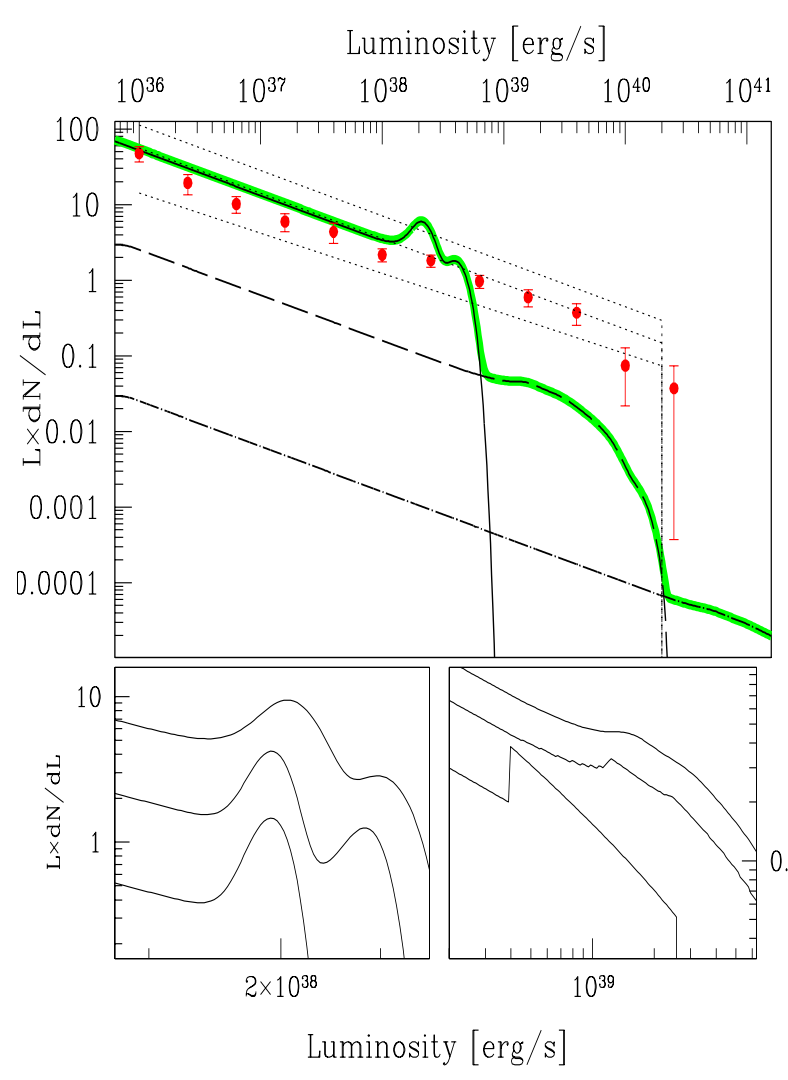

Figure 6.6.: The upper main figure shows the contributions of neutron stars (thin solid line), stellar (dashed line) and intermediate (dot-dashed line) mass BHs to the differential luminosity function. The thick grey solid line is the total differential luminosity function. For details of the parameters see discussion in the text. The figure in the lower left shows the luminosity around the Eddington limit for a NS. The luminosity functions shown include the simplest assumption that all systems with accretion rates above Eddington radiate at the Eddington luminosity (bottom), two effects allowing superEddington luminosities (middle), and additionally a $20 \%$ uncertainty in the distance estimate (top). The curves are scaled for clarification. The figure in the lower right shows the luminosity around the Eddington limits 0.0 for $3-20 \mathrm{M}_{\odot}$ BHs. The luminosity functions shown include no effect (bottom), and two effects allowing super-Eddington luminosities (middle) and additionally a $20 \%$ uncertainty in the distance estimate (top). The dotted lines denote the uncertainty due to SFR of a factor of 2 .

of mass transfer rates to X-ray luminosities.

The probability distribution of NS masses was chosen to be a Gaussian distribution with a mean of $1.4 \mathrm{M}_{\odot}$ and a $\sigma$ of $0.2 \mathrm{M}_{\odot}$. The mass distribution of BHs was chosen to be a power law with a slope of 1.1. These numbers are similar to results of theoretical computations performed by Fryer \& Kalogera (2001). The mass distribution for BHs is bimodal, for stellar mass black holes it ranges from 3-20 $\mathrm{M}_{\odot}$, and secondly, we include intermediate mass $\mathrm{BHs}$ ranging from $10^{2}-10^{5} \mathrm{M}_{\odot}$. We made the simple assumption that their mass distribution has the same slope as for stellar mass BHs.

Normalisations for the probability distributions were chosen such that the number of stellar mass BHs is a factor of 20 smaller than the number of NSs. This is roughly the ratio observed for HMXBs in our Galaxy (Portegies Zwart \& Yungelson 1998; Iben et al. 1995; Grimm et al. 2002). However the ratio of stars with $M>25 M_{\odot}$, BH progenitors, to stars with $25 M_{\odot}>M>8 M_{\odot}$, NS progenitors, is close to $1 / 2$ according to the Salpeter IMF. Therefore in principle the stellar mass BH curve in Fig. 6.6 might be much closer to the NS curve. The number of intermediate mass BHs is assumed to be a factor of 100 less than the number of stellar mass BHs in HMXBs. 
The probability distribution of mass transfer rates in binary systems is set to be a power law with a slope of -1.6, reproducing the observed luminosity function of HMXBs assuming a linear relation between luminosity and mass accretion rate. The limits are 0.1 to $10^{7}$ in units of $10^{16} \mathrm{~g} / \mathrm{s}$. Mass transfer was assumed to be conservative over the whole range, i.e. no mass is lost from the system except for super-Eddington sources and wind accretion. The formulae for conversion of mass accretion rate to X-ray luminosity are

$$
L=\eta \cdot \dot{M}_{\text {accretion }} \cdot c^{2},
$$

where $\eta=0.1$ for BHs and $\eta=0.15$ for NSs. The mass loss rate from the normal star has no strict limit, however the X-ray luminosity reaches a maximum at the Eddington luminosity and objects with much higher mass accretion rate will end up at the Eddington luminosity introducing a peak in the luminosity function.

For illustration we present two sub-figures in Fig. 6.6 to show the evolution from sharp features to a smoother curve with the introduction of smearing effects on the luminosity which is shown in the main part of the figure. The first effect is He-accretion when the HMXB is fed by a helium rich star which we take to be the case in about $10 \%$ of the sources. Secondly, in the case of BHs a quasi-flat accretion disk with an electron scattering atmosphere (Sobolev 1949; Chandrasekhar 1950) radiates according to $(1+2.08 \cdot \cos (i)) \cos (i)$ where $i$ is the inclination angle, producing 2.6 times higher flux in the direction perpendicular to the disk plane than average (Shakura \& Sunyaev 1973). Sunyaev \& Titarchuk (1985) confirmed that this ratio is similar or higher for radiation comptonised in the accretion disk. For slim disks (Paczynsky \& Wiita 1980) this ratio should be even higher. Moreover to demonstrate the influence of distance uncertainties we assumed a variation in distances of $20 \%$. All these effects together give a considerably smoother curve and permit up to 6 times higher luminosities.

These are only the most simple effects that permit to surpass the Eddington limit. Of course other more sophisticated models like jet emission (Körding et al. 2002) or beamed emission (King et al. 2001) or models taking into account strong magnetic fields in X-ray pulsars (Basko \& Sunyaev 1976) also can be employed to explain the observed luminosity function.

\section{Wind driven accreting systems}

Our experience with HMXBs in our Galaxy and LMC shows that in many sources accretion happens via capture from a strong stellar wind (Cen X-3, Cyg X-1, 4U 1700+37, 4U 090040, and possibly SMC X-1, LMC X-1 and LMC X-4) As we see the majority of Galactic HMXBs are fed by stellar wind accretion. There is a very important difference between wind accretion onto NSs and BHs. The capture radius, $r_{\text {capture }}=\frac{2 G M}{v_{0}^{2}}$, is proportional to the mass of the accreting object and therefore in similar systems BHs should have $M^{2}$ times larger accretion rates than NSs for the same wind parameters. The dependence of the Roche geometry on the mass ratio make the dependence on $\mathrm{M}_{B H}$ a little weaker.

$$
\dot{M}_{\text {capture }} \propto \dot{M}_{\text {wind }} \cdot\left(\frac{M_{B H}}{M_{N S}}\right)^{\beta},
$$


where $\beta$ is between 1.5 and 2 . This reason might increase the relative $\mathrm{BH}$ contribution to the luminosity function in star forming galaxies. It is important that

$$
\frac{\dot{M}_{\text {capture }} \cdot \eta \cdot c^{2}}{L_{E d d}} \propto \dot{M}_{\text {wind }} \cdot M_{B H}^{\beta-1} .
$$

For $\beta>1$ it is preferable for BHs to have higher luminosities than for NSs.

\section{Comparison of simulated and observed luminosity function}

The discrepancy between the observed luminosity function in the right panel of Fig. 6.3 and our simple model in Fig. 6.6 is obvious. We do not see features in the observed differential luminosity function in the vicinity of $L_{E d d}$ for NSs, neither a peak $\frac{\Delta L}{L} \sim O(2)$ nor a sharp decline at $L>L_{E d d}$ as in the model luminosity function. Furthermore our model luminosity function lacks sources in the luminosity range $10^{39}-10^{40} \mathrm{erg} / \mathrm{s}$. It seems we should assume that accreting stellar mass BHs in star forming regions are more abundant than in the Milky Way.

It is important to note that having all our corrections we are getting objects close to the limit of maximum luminosity of the observed luminosity functions.

In Fig. 6.6 is plotted the total accretion luminosity whereas CHANDRA observes only in the range from 1-10 keV. However X-ray pulsars emit the bulk of their luminosity in the range from 20-40 keV. This effect may further decrease the importance of the peak at $2 \cdot 10^{38} \mathrm{erg} / \mathrm{s}$. Since in elliptical galaxies old X-ray binaries with weak magnetic fields, thus having much softer spectra than X-ray pulsars, should dominate the population one should expect the importance of the peak to be larger in ellipticals.

Our simple analysis demonstrates how difficult it is to construct a very smooth luminosity function with the same slope over a broad luminosity range, $10^{35}-10^{40} \mathrm{erg} / \mathrm{s}$, and without sharp features in the vicinity of Eddington luminosities. Because so many different processes are involved in different parts of this huge luminosity range. Our universal luminosity function based on CHANDRA, ASCA and RXTE data has no strong features. The absence of features around the Eddington luminosity for NSs should be explained but it is also necessary to explain the absence of the abrupt change in the luminosity function at higher luminosities when less numerous BHs dominate the luminosity function.

The most obvious shortcomings of this naive model are the mass distributions of BHs and $\mathrm{NSs}$, the normalisations for BHs, especially for intermediate mass BHs, and the assumptions of conservative mass transfer and that all super-Eddington sources radiate at Eddington luminosity in X-rays. It is also very difficult to assume that intermediate mass BHs form a continuous mass function with stellar mass BHs without a strong break around 20-50 $\mathrm{M}_{\odot}$. They should have their own luminosity function with different normalisation and slope. Another problem is connected with the formation of binaries with normal stars feeding intermediate mass BHs and making them bright X-ray sources. The observation of HMXBs in other galaxies will allow to put constraints on the combination of these parameters. 
luminosity function (ULF) is connected with the interpretation of the following experimental facts:

- RXTE/ASM, ASCA and CHANDRA give us information about the low luminosity part of the ULF $\left(L_{X}<10^{38} \mathrm{erg} / \mathrm{s}\right)$ based on the Milky Way, SMC and NGC 1569.

- CHANDRA data on the other galaxies in Table 5.1 give information about the high luminosity part of the ULF $\left(L_{X}>10^{38} \mathrm{erg} / \mathrm{s}\right)$.

- UV, FIR and radio methods of SFR determination in both local and more distant samples of galaxies have significant systematic uncertainties, see Table 5.3.

To resolve these uncertainties arising very close to the Eddington luminosity for a NS we need to additional data permitting to get the slope of the luminosity function in Antennaetype galaxies at luminosities significantly below $10^{38} \mathrm{erg} / \mathrm{s}$. Furthermore we need to increase the sample of nearby galaxies where we can extend the luminosity function well above $10^{38}$ $\mathrm{erg} / \mathrm{s}$. Only this will give full confidence that there is no change in the normalisation in the ULF near $10^{38} \mathrm{erg} / \mathrm{s}$.

\subsubsection{Further astrophysically important information}

The good correlation between SFR and total X-ray luminosity due to HMXBs and the total number of HMXBs can obviously become a powerful and independent way to measure SFR in distant galaxies. In addition, this correlation is providing us with further astrophysically important information:

- These data are showing that NSs and BHs are produced in star forming regions very efficiently and in very short time, confirming the main predictions of stellar evolution.

- The luminosity function of HMXBs does not seem to depend strongly on the trigger of the star formation event which might be completely different for the Milky Way and e.g. the Antennae where it is the result of tidal interaction of two galaxies.

- The good agreement of the X-ray luminosity - SFR relation of HDF galaxies with the theoretical prediction proves that the HMXB formation scenario at high redshifts does not differ significantly from nearby HMXB formation.

- The luminosity function provides information that neutron stars and BHs have a similar distribution of accretion rates in all galaxies of the sample available for study today.

- The luminosity function of HMXBs does not seem to depend strongly on the chemical abundances in the host galaxy.

- The existence of well separated X-ray sources is a way to look for small satellites of massive galaxies, like SMC. 
The integral X-ray luminosity and X-ray source counts are unique sources of information on binaries in distant galaxies. Other methods of investigation of SFR (UV, IR, radio) rely on the luminosity distribution and number of the brightest stars, without a significant dependence on the amount of binaries in a high mass star population. On the other hand the existence of an observed population of HMXBs in another galaxy is possible only in the case if there are conditions for formation of close binaries with certain mass loss from a normal companion and efficient capture of out-flowing stellar wind or Roche lobe overflow by an accreting object. Detailed observations of X-ray sources in our own Galaxy have shown how small the allowed parameter space is - this is the reason why the number of X-ray sources in the Galaxy is so small (Illarionov \& Sunyaev 1975) in comparison with the total number of NSs and BHs and the total number of $\mathrm{O}$ and $\mathrm{B}$ stars. Therefore:

- The existence of a universal luminosity function of HMXBs proves that the formation of close massive X-ray binaries and their distribution on mass ratio, separation and mass exchange rate is similar in all regions of active star formation up to redshifts $\mathrm{z} \sim 1$. 


\subsection{Collective luminosity of a population of discrete sources}

Consider a population of discrete sources of a certain type (e.g. X-ray binaries) in a galaxy. For the sake of this discussion we will be referring to high mass X-ray binaries in star forming galaxies since we have applied this consideration to this kind of sources in the previous chapter. Suppose that the luminosity function of HMXB sources in each galaxy is

$$
\frac{d N}{d L}=A(S F R) \times f(L),
$$

where the shape $f(L)$ is the same for all galaxies and the normalisation $A$ is proportional to the star formation rate (SFR) of a galaxy. In this case the expectation value for the total number of sources in a galaxy,

$$
<N_{\text {total }}>=\int_{0}^{+\infty} \frac{d N}{d L} d L \propto A(S F R),
$$

is directly proportional to its star formation rate. Apart from effects of counting statistics, the number of HMXB sources found in any arbitrarily chosen galaxy will be equal to the above expectation value. The skewness of the Poisson distribution is relatively unimportant in this context as soon as the total number of sources exceeds $\sim 5-10$.

The problem considered here is the behaviour of the total (collective) luminosity of the high mass X-ray binaries in a galaxy,

$$
L_{\text {total }}=\sum_{k=1}^{k=N} L_{k}
$$

as a function of the star formation rate of the parent galaxy.

At first glance the collective luminosity of HMXB sources in a galaxy with a known star formation rate is given by its expectation value $\left\langle L_{\text {total }}\right\rangle$ which can easily be derived integrating the luminosity function:

$$
<L_{\text {total }}>\int_{0}^{+\infty} L \frac{d N}{d L} d L \propto A(S F R) .
$$

Hence one might expect that the collective luminosity of a HMXB population in an arbitrarily chosen galaxy should be directly proportional to the galaxy's star formation rate. However, the problem involves some subtleties related to the statistical properties of the power law distribution of the sources, which appear not to have been recognised previously (at least in astrophysical context).

In the following we assume that the probability distribution is a power law with a slope $\alpha$ and a normalisation $A$, which for the sake of the argument is proportional to the star formation rate (SFR). The main result, however is valid also for other, highly skewed probability distributions. 
The main surprise of the study presented here is that in the low SFR regime (small values of $A$, i.e. "small" number of sources) the relation between SFR of the parent galaxy and collective luminosity of its HMXB sources is non-linear - with increase of the star formation rate the luminosity grows faster that linear. The relation becomes linear only for sufficiently high star formation rates, when the total number of sources becomes sufficiently large.

The non-linear $L_{X}-S F R$ dependence is caused by the non-Gaussianity and large degree of the skewness of the probability distribution of the collective luminosity $p\left(L_{\text {total }}\right)$. The effect of the skewness of $p\left(L_{\text {total }}\right)$ is that the most probable value of the total luminosity of the HMXB population observed in an arbitrarily chosen galaxy (the mode of the $p\left(L_{\text {total }}\right)$ distribution) might deviate significantly from the expectation value given by Eq. 6.17. Of course the mean value of $L_{\text {total }}$ (i.e. averaged over many galaxies with similar star formation rates) equals to the expectation value given by Eq.6.17 and obeys the linear dependence on the star formation rate. This equality is maintained due to the outliers whose luminosities exceed significantly both the most probable and the expectation values. In addition these outliers will result in enhanced and non-symmetric dispersion in the non-linear (low SFR) regime.

The position of the break in the $L_{X}-S F R$ relation, i.e. the change from the non-linear to linear regime, depends on the parameters of the luminosity function and can occur for a total number of sources as large as $\gtrsim 10^{2}-10^{3}$ and larger.

Interestingly the entire existence of a linear regime in the $L_{X}-S F R$ relation is a direct consequence of the existence of a cut-off in the luminosity function. For a sufficiently flat luminosity function, a power law slope $1<\alpha<2$, the collective luminosity of the sources grows faster than linear because not only more but also brighter sources contribute to the total luminosity as the star formation rate increases. Only in the presence of a maximum possible luminosity for the sources, $L_{c}$, for instance the Eddington luminosity of a neutron star, the regime can be reached, when $N\left(>L_{c}\right)$ becomes larger than unity and subsequent increases of the star formation rate result in a linear growth of the total luminosity.

We used high mass X-ray binary populations in star forming galaxies as an example in the above discussion. The effect considered here is however of broader general interest and might work in many different situations related to computing/measuring integrated properties of a limited number of discrete sources.

\subsubsection{Analytical treatment}

We consider a population of sources with a power law luminosity function between $L_{1}$ and $L_{2}$ :

$$
\frac{d N}{d L}=A \cdot L^{-\alpha}
$$

The expectation value of the total luminosity is:

$$
<L_{\text {total }}>=\frac{A}{2-\alpha}\left(L_{2}^{2-\alpha}-L_{1}^{2-\alpha}\right) .
$$


For fixed $A$ and $\alpha$ the probability to have $n$ sources in any given realization obeys the Poisson distribution $P(n, \mu)=\frac{\mu^{n}}{n !} e^{-\mu}$ with mean value $\mu$ of

$$
\mu=\frac{A}{1-\alpha}\left(L_{2}^{1-\alpha}-L_{1}^{1-\alpha}\right) .
$$

As the number of sources must be integer, the probability distribution for the total luminosity of the source population $L_{\text {total }}$ is given by

$$
p\left(L_{\text {total }}, A, \alpha\right)=\sum_{k=0}^{k=\infty} P(k, \mu) p_{n}\left(L_{\text {total }}, \alpha\right),
$$

where $p_{n}\left(L_{\text {total }}, \alpha\right)$ is the probability distribution of the total luminosity of $n$ sources, where $n$ is fixed.

In the majority of practically interesting cases, the total number of sources is sufficiently large, $\mu \gg 1$, and the summation over the Poisson distribution in Eq.6.22 can be neglected.

$$
p\left(L_{\text {total }}, A, \alpha\right) \approx p_{n}\left(L_{\text {total }}, \alpha\right), n=\mu \gg 1,
$$

where $n=\mu$ is given by Eq.6.20.

For $n$ sources with the luminosities distributed according to Eq.6.18, the probability distribution for the total luminosity $L_{\text {total }}=\sum_{k=1}^{k=n} L_{k}$ can be calculated recursively as a sequence of convolutions:

$$
p_{n}\left(L_{t o t a l}, \alpha\right)=\int_{0}^{\infty} p_{n-1}(x, \alpha) \cdot p_{1}\left(L_{t o t a l}-x, \alpha\right) d x
$$

where $p_{1}(x, \alpha)$ is the probability distribution for the luminosity of one source, which is given by Eq.6.18 with appropriate normalisation:

$$
p_{1}(L, \alpha)=\frac{1-\alpha}{L_{2}^{1-\alpha}-L_{1}^{1-\alpha}} L^{-\alpha}
$$

A more convenient method for the computation of $p_{n}\left(L_{t o t a l}, \alpha\right)$ is to use the characteristic function of $p_{1}$ and the convolution theorem:

$$
\begin{array}{rc}
\hat{p_{n}} & ={\hat{p_{1}}}^{n} \\
\hat{p_{1}}(t) & =\int_{-\infty}^{+\infty} p_{1}(x) \exp ^{i t x} d x \\
p_{n}(x) & =\int_{-\infty}^{+\infty} \hat{p_{n}}(t) \exp ^{-i t x} d x
\end{array}
$$

The probability distribution $p_{n}\left(L_{\text {total }}, \alpha\right)$ for various values of $\alpha$ and $n$ are shown in Fig. 6.7. For convenience the abscissa on these plots is the average luminosity $L_{\text {total }} / n$.

As is obvious from Fig. 6.7 the skewness of the probability distribution $p_{n}\left(L_{\text {total }}, \alpha\right)$ leads to a deviation of its most probable value (the mode of the distribution) $\tilde{L}_{\text {total }}$ from the expectation value $\left\langle L_{\text {total }}\right\rangle$, indicated in each panel by the vertical dashed line. The 

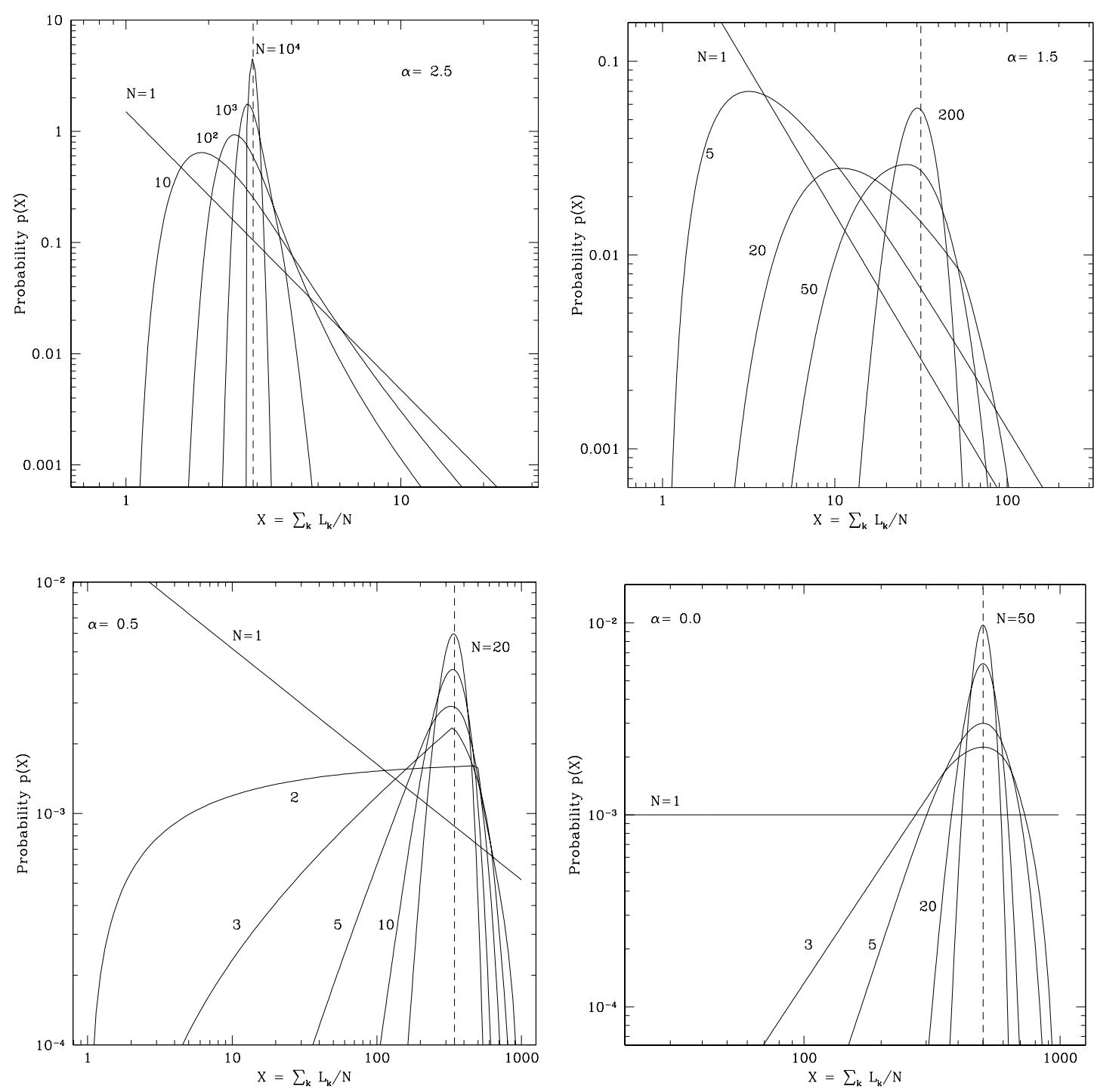

FiguRe 6.7.: Probability distributions of the average luminosity of $N$ discrete sources with a luminosity function $d N / d L=A \cdot L^{-\alpha}$ for various values of the luminosity function slope $\alpha$. The ratio of cut-off luminosities was fixed at $\frac{L_{2}}{L_{1}}=10^{3}$. The value of $\alpha$ is indicated in each panel. Each curve is marked according to the number of sources $N$. The vertical dashed line shows the expected value of the sample averaged luminosity. Note that: (i) The sample average is observed for arbitrarily small number of sources, i.e. $\int p(L) L d L / N$ is always equal to the sample average; (ii) For small values of $N$ the maximum probable value of luminosity deviates from the sample average; (iii) The skewness of the probability distribution at small $N$ results in relatively infrequent large values of the average luminosity. For illustration the probability distributions for flat, $\alpha=0$, are shown in the bottom-right panel. As expected in this case the maximum probable luminosity and the sample average coincide exactly for any $N$. 
effect is strongest for $\alpha>1$ and vanishes for sufficiently shallow luminosity functions with $\alpha<1$. This can be understood as in the case of $\alpha<1$ both the total number of sources and the total luminosity are defined by the upper integration limit (high luminosity cut-off) $L_{2}$. Correspondingly, e.g. for $\alpha=0.5$, the maximum of the probability distribution $p_{n}\left(L_{\text {total }}\right)$ does not deviate noticeably from the expectation value $\left\langle L_{\text {total }}\right\rangle$. For illustration we also show the case of the flat luminosity function, $\alpha=0$, in which case $\tilde{L}_{\text {total }}$ coincides with $<L_{\text {total }}>$. Naturally for any value of $\alpha$ the distribution $p_{n}\left(L_{\text {total }}\right)$ becomes Gaussian in the limit of $n \rightarrow \infty$ in accord with the Central Limit Theorem.

The dependence of the most probable value of the total luminosity $\tilde{L}_{\text {total }}$ on the total number of sources $n$ for different values of $\alpha$ and the ratio $\frac{L_{2}}{L_{1}}$ is shown in Figs. 6.9 and 6.8. Interestingly for a slope of the luminosity function in the range $1<\alpha<2$ the dependence of $\tilde{L}_{\text {total }}$ vs. $n$ shows a relatively sharp break, separating the non-linear part from the linear. The position of the break in the $\tilde{L}_{\text {total }}-n$ plane depends on the slope of the luminosity function and the ratio of the maximum to minimum luminosity $\frac{L_{2}}{L_{1}}$. These dependences as well as the asymptotic behaviour of $\tilde{L}_{\text {total }}$ will be considered in the following.

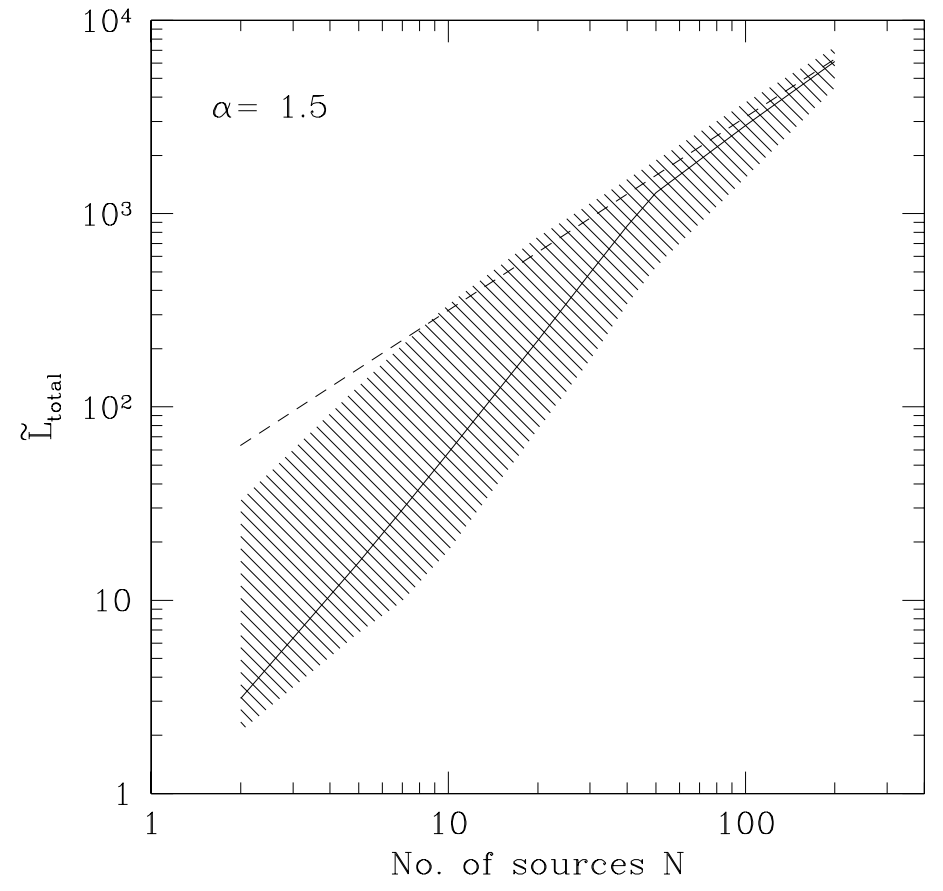

Figure 6.8.: The most probable value of the total luminosity $\tilde{L}_{\text {total }}$ and the $67 \%$ probability area versus number of sources for a slope $\alpha=1.5$ and $\frac{L_{2}}{L_{1}}=10^{3}, L_{1}=1$. The results are from the exact calculation using Eq.(6.27). The behaviour of $\tilde{L}_{\text {total }}$ is shown by the solid line, the dashed area corresponds to the $67 \%$ probability area, and the dashed line shows the linear behaviour of $\tilde{L}_{\text {total }}=<L_{\text {total }}>$.

As was mentioned above, the mean luminosity defined as an average of $L_{\text {total }}$ over source samples with the same luminosity function (e.g. average of the total luminosity of many star-forming galaxies with the same value of star formation rate) equals to the expectation value $\left\langle L_{\text {total }}\right\rangle$ for any number of sources, i.e. is always proportional to the normalisation of the luminosity function. This equality is achieved due to the existence of outliers, having a value of $L_{\text {total }}$ significantly exceeding both $\tilde{L}_{\text {total }}$ and $\left\langle L_{\text {total }}\right\rangle$, in accordance 
with the skewness of the probability distribution $p_{n}\left(L_{\text {total }}\right)$ for small $n$. This leads naturally to enhanced and asymmetric dispersion of the observed values of $L_{\text {total }}$ in the non-linear regime, as illustrated in Fig. 6.8.
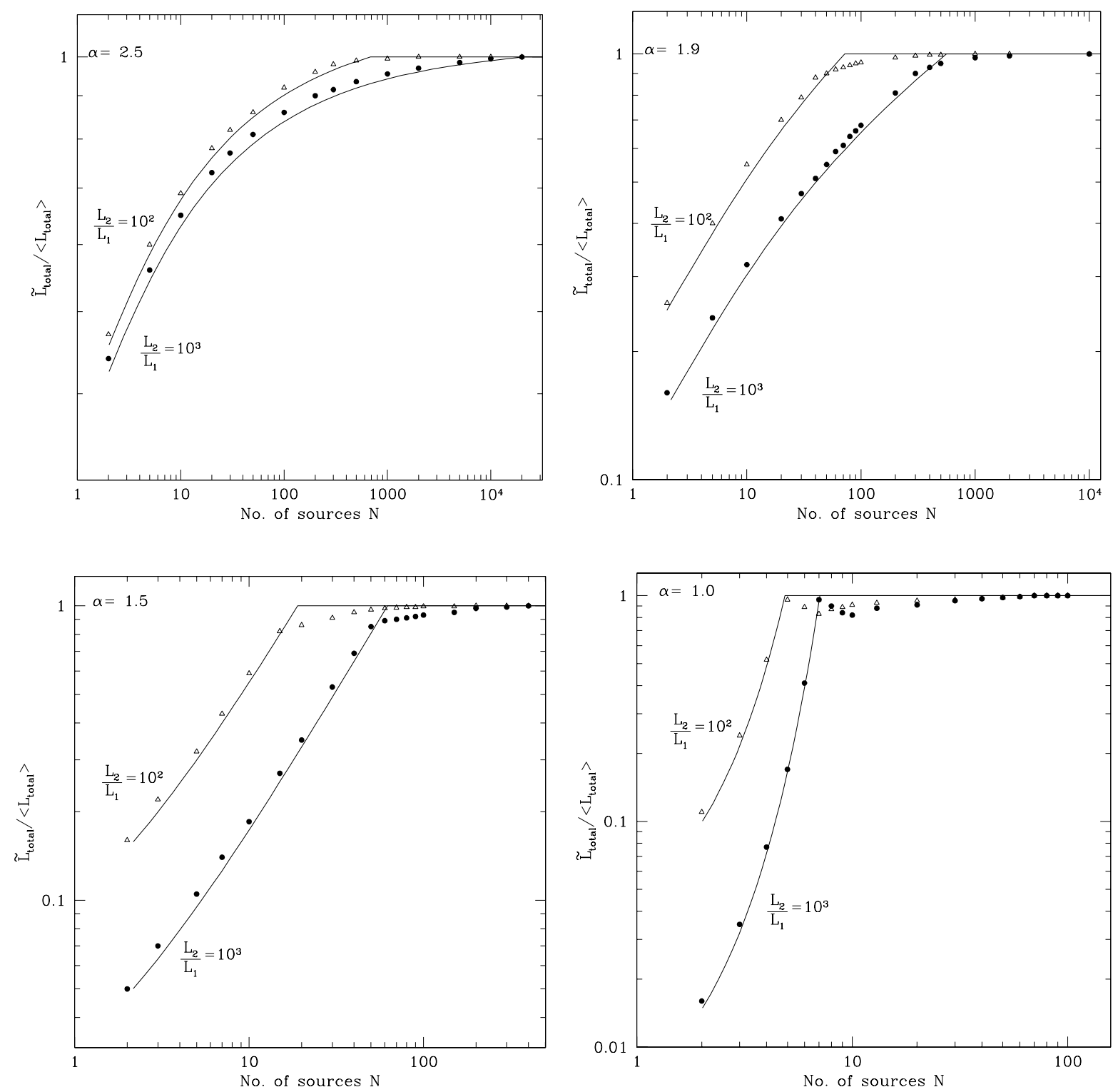

FiguRE 6.9.: The ratio of the most probable value of total luminosity $\tilde{L}_{\text {total }}$ to its expectation value $L_{\text {total }}$ versus number of sources for different values of the luminosity function slope $\alpha$ and the ratio $\frac{L_{2}}{L_{1}}$. The results of the exact calculation using Eq. 6.27 are shown by the dots and triangles. The solid line shows the approximate relation from Eqs. 6.31 and 6.36.

The following simple arguments lead to an approximate expression for the most probable 
luminosity.

For a given probability distribution of the luminosity of one sources $p_{1}(L)$ (Eq.6.24) and a given number of sources in the sample, the probability distribution for the maximum luminosity, $L_{\max }$, in the sample is defined, apart from the normalisation, by:

$$
p_{\max }\left(L_{\max }\right) \propto\left[p_{1}\left(L<L_{\max }\right)\right]^{n-1} p_{1}\left(L_{\max }\right),
$$

where $p_{1}\left(L<L_{\max }\right)$ denotes the cumulative probability

$$
p_{1}\left(L<L_{\max }\right)=\int_{0}^{L_{\max }} p_{1}(L) d L .
$$

The probability distribution $p_{\max }\left(L_{\max }\right)$ is shown in Fig. 6.10 and illustrates the intuitively obvious fact that, if the number of sources is sufficiently small, the brightest sources would most likely not reach the highest possible value of $L_{2}$. Similarly the probability distribution of the minimum luminosity in the sample is:

$$
p_{\text {min }}\left(L_{m i n}\right) \propto\left[p_{1}\left(L>L_{m i n}\right)\right]^{n-1} p_{1}\left(L_{m i n}\right) .
$$

Contrary to $p_{\max }\left(L_{\max }\right), p_{\min }\left(L_{\min }\right)$ declines steeply at $L>L_{1}$ for any $n$ - as the $p_{1}(L)$ distribution is a decreasing function with luminosity. With an accuracy sufficient for this approximation it can be assumed that $p_{\min }\left(L_{\min }\right)=\delta\left(L_{\min }-L_{1}\right)$, i.e. $L_{\text {min }}=L_{1}$.

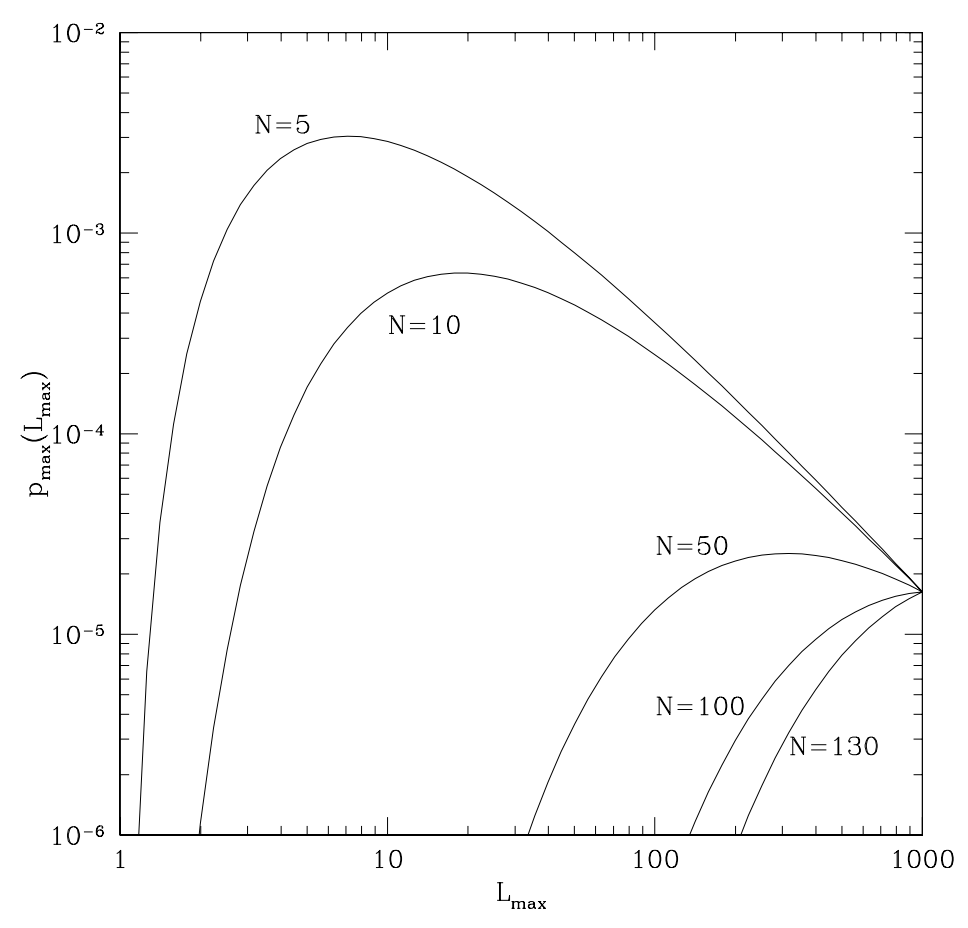

Figure 6.10.: Probability distribution of the maximum luminosity in a sample for a luminosity function with a slope $\alpha=1.5$ and $L_{1}=1$, $L_{2}=1000$ for various values of the number of sources in the sample $N$. The probability is computed according to Eq. 6.28. Evidently for small number of sources it is not likely to observe the brightest source at the maximum possible luminosity $L_{2}$. 
The total luminosity of $n$ sources distributed according to the power law with slope $\alpha$ between $L_{1}$ and $L_{\max }$ is given by

$$
L_{\text {total }}\left(L_{\max }, \alpha, n\right)=n \cdot \frac{1-\alpha}{2-\alpha} \cdot \frac{L_{\max }^{2-\alpha}-L_{1}^{2-\alpha}}{L_{\max }^{1-\alpha}-L_{1}^{1-\alpha}} .
$$

Knowing the probability distribution for $L_{\max }$ the probability distribution $p_{n}\left(L_{\text {total }}\right)$ can be calculated as:

$$
p_{n}\left(L_{\text {total }}\right) \approx p_{\max }\left[L_{\max }\left(L_{\text {total }}\right)\right] \cdot\left(\frac{d L_{\text {total }}}{d L_{\max }}\right)^{-1}
$$

where $L_{\text {max }}\left(L_{\text {total }}\right)$ is the inverse function to Eq. 6.31 .

The maximum probable value value of the total luminosity of a sample of $n$ sources is defined by the condition

$$
\frac{d p_{n}\left(L_{t o t a l}\right)}{d L_{\text {total }}}=0
$$

With Eqs.6.28, 6.31 and 6.32 the above equation can be transformed to:

$$
(\alpha-2) \xi^{2 \alpha}-(\alpha-2)(1+\alpha / n+\alpha n) \xi^{1+\alpha}+(\alpha-1)^{2}(1+n) \xi^{\alpha}+[1+(\alpha-1) n] \xi^{2}=0
$$

with

$$
\xi=\frac{L_{\max }}{L_{1}}
$$

or, equivalently,

$$
n=\frac{(\alpha-2) \xi^{2 \alpha}+\left(2+\alpha-\alpha^{2}\right) \xi^{1+\alpha}+(\alpha-1)^{2} \xi^{\alpha}-\xi^{2}}{(\alpha-1)\left[(\alpha-2) \xi^{1+\alpha}-(\alpha-1) \xi^{\alpha}+\xi^{2}\right]}
$$

Because of the simplifying assumption $p_{\min }\left(L_{\min }\right)=\delta\left(L_{\min }-L_{1}\right)$ the probability distribution defined by Eqs.6.28, 6.31 and 6.32 is valid only for $L_{\text {total }}<<L_{\text {total }}>$. Indeed this assumption implies that the maximum value of $L_{\text {total }}$ is achieved when the luminosities of the faintest and brightest sources in the sample are $L_{\min }=L_{1}$ and $L_{\max }=L_{2}$ and, therefore it can not exceed $<L_{\text {total }}>$. This however is sufficient for our purpose as $L_{\max } \sim L_{2}$ corresponds to the break in the $\tilde{L}_{\text {total }}-n$ relation (Figs. 6.9 and 6.8 ) above which $\tilde{L}_{\text {total }}=<L_{\text {total }}>$.

The dependence of the maximum probable total luminosity of the sample $\tilde{L}_{\text {total }}$ on the number of sources $n$ can be conveniently computed parametrically using Eqs. 6.36 and 6.31. The practical recipe is for a set of values from $L_{\max }, L_{1}<L_{\max } \leq L_{2}$, to compute $n\left(L_{\text {max }}\right)$ from Eq.6.36 and $L_{\text {prob }}(n, \alpha)=L_{\text {total }}\left(L_{\text {max }}, n, \alpha\right)$ from Eq.6.28. The pairs of values $\left(n, L_{\text {total }}\right)$ define the $\tilde{L}_{\text {total }}-n$ relation before and up to the break, whereas above the break $\tilde{L}_{\text {total }}=<L_{\text {total }}>$ and can be computed from Eq. 6.31 with $L_{\text {max }}=L_{2}$ and $n>n_{\text {break }}$.

The approximation defined by Eqs. 6.36 and 6.31 is compared with the results of the exact calculation in Fig. 6.9. It is accurate to within $\sim$ several per cent everywhere, except the break region where its accuracy is $\sim 10-20 \%$. 
Using the approximate solution for $\tilde{L}_{\text {total }}$ obtained above we consider asymptotical the behaviour of the $\tilde{L}_{\text {total }}-n$ relation, concentrating on the most interesting case of $\alpha>1$ and in the limit of $L_{1} \ll L_{2}$.

The number of sources $n_{\text {break }}$, corresponding to the break in the $\tilde{L}_{\text {total }}-n$ relation, can be obtained from Eq. 6.36, substituting $\xi=\frac{L_{2}}{L_{1}}$ and using the fact that $\xi \ll 1$ :

$$
n_{b r e a k} \approx \frac{1}{\alpha-1} \cdot\left(\frac{L_{2}}{L_{1}}\right)^{\alpha-1}
$$

Expressed in terms of the normalisation $A$ of the luminosity function Eq.6.18 it is:

$$
A_{\text {break }} \approx L_{2}^{\alpha-1}
$$

As is intuitively expected, the break position expressed in terms of the normalisation of the luminosity function does not depend on the low luminosity cut-off $L_{1}$ and is defined only by the slope and the high luminosity cut-off of the luminosity function. The total luminosity at the break, however, depends on the low luminosity cut-off for steep luminosity function with $\alpha>2$ :

$$
L_{b r e a k}^{\text {prob }} \approx\left\{\begin{array}{l}
\frac{L_{2}}{2-\alpha} \text { if } 1<\alpha<2 \\
\frac{L_{2}}{\alpha-2} \cdot\left(\frac{L_{2}}{L_{1}}\right)^{\alpha-2} \text { if } \alpha>2
\end{array}\right.
$$

This can be understood as, the total luminosity for $\alpha>2$ is defined by the sources near the low luminosity cut-off. 


\section{Summary}

We studied the population of X-ray binaries in the Milky Way in previously unachievable detail thanks to the great advances in observations of these sources in all wavebands. A more detailed understanding of the properties of X-ray binaries will play an important role in the investigation of the X-ray binary population in other galaxies. The most important results of our analysis are the following:

- In good agreement with theoretical expectations and earlier results (van Paradijs \& White 1995; White \& van Paradijs 1996; Koyama et al. 1990; Nagase 1989) we found significant differences in the spatial (3-D) distribution of high and low mass X-ray binaries. HMXBs are more concentrated towards the Galactic Plane with a vertical scale height of $150 \mathrm{pc}$, tend to avoid the Galactic Bulge and central $\sim 3-4 \mathrm{kpc}$ of the Galaxy and show clear signatures of the spiral structure. The distribution of LMXB sources, on the contrary, peaks strongly at the Galactic Bulge and shows a pronounced minimum at $\sim 3-4 \mathrm{kpc}$. Some signatures of the Galactic spiral structure are also present. The vertical distribution of LMXB sources is significantly broader, with a scale height of $410 \mathrm{pc}$.

- We constructed the long-term averaged $\log (\mathrm{N})-\log (\mathrm{S})$ distribution of high and low mass X-ray binaries in the $2-10 \mathrm{keV}$ energy range using the data of the ASM instrument aboard RXTE from 1996-2000 to the limiting sensitivity of $\approx 6.4 \cdot 10^{-11} \mathrm{erg}$ $\mathrm{s}^{-1} \mathrm{~cm}^{-2}$. The $\log (\mathrm{N})-\log (\mathrm{S})$ distribution of HMXBs is well described by a simple power law with a slope of the differential distribution of $1.61_{+0.12}^{-0.14}$ down to a flux limit of $\approx 6.4 \cdot 10^{-11} \mathrm{erg} \mathrm{s}^{-1} \mathrm{~cm}^{-2}$. The differential $\log (\mathrm{N})-\log (\mathrm{S})$ distribution of LMXBs has a slope of $-1.2 \pm 0.06$ and requires a high-flux cutoff at $\sim 110$ ASM cts $\mathrm{s}^{-1}, \approx 3.5 \cdot 10^{-8} \mathrm{erg} \mathrm{s}^{-1} \mathrm{~cm}^{-2}$. A comparison with data of the ASCA Galactic Ridge Survey (Sugizaki et al. 2001) which covered $\sim 40$ square degrees with $\sim 100$ times better sensitivity did not reveal any evidence of significant departures of the $\log (\mathrm{N})-\log (\mathrm{S})$ from an extrapolation of the ASM data down to $\approx 5 \cdot 10^{-13} \mathrm{erg} \mathrm{s}^{-1}$ $\mathrm{cm}^{-2}$.

- Using the source distances available and assuming a model for the volume density distribution we constructed luminosity functions for HMXBs and LMXBs in the 2-10 $\mathrm{keV}$ energy range. The sensitivity limit of the ASM catalogue allows one to study the XRB luminosity functions down to a luminosity of $\sim 2 \cdot 10^{35} \mathrm{erg} \mathrm{s}^{-1}$. The differential luminosity functions can be described by a power law with slopes of 1.64 and 1.27 for HMXBs and LMXBs respectively. For LMXB sources a cut-off at $\sim 2.7 \cdot 10^{38} \mathrm{erg}$ $\mathrm{s}^{-1}$ is required. The HMXB data are insufficient to detect a high luminosity cut-off 
above $\sim$ few $\times 10^{36} \mathrm{erg} \mathrm{s}^{-1}$. A comparison with the data of ASCA Galactic Ridge Survey did not find evidence for significant departures from these power laws down to luminosities of $\sim 10^{34} \mathrm{erg} \mathrm{s}^{-1}$.

- The complete catalogue of our sample of X-ray binaries is given in the appendix, chapter 8. Properties of the brightest sources are summarised in Tables 3.2.2, 3.2.2, 3.2.3.

- The integrated luminosity of X-ray binaries in the Milky Way in the $2-10 \mathrm{keV}$ band averaged over 1996-2000 is $\sim 2-3 \cdot 10^{39} \mathrm{erg} \mathrm{s}^{-1}$ to which LMXB sources contribute $\sim 90 \%$. Normalised to the Galactic stellar mass and the Galactic star formation rate, the integrated luminosity of LMXBs $\left(\sim 2.5 \cdot 10^{39} \mathrm{erg} \mathrm{s}^{-1}\right)$ and HMXBs $\left(\sim 2 \cdot 10^{38}\right.$ $\mathrm{erg} \mathrm{s}^{-1}$ ) correspond to $\sim 5 \cdot 10^{28} \mathrm{erg} \mathrm{s}^{-1} \mathrm{M}_{\odot}^{-1}$ and $\sim 5 \cdot 10^{37} \mathrm{erg} \mathrm{s}^{-1} /\left(\mathrm{M}_{\odot} \mathrm{yr}^{-1}\right)$, respectively. The total number of the X-ray binaries brighter than $2 \cdot 10^{35} \mathrm{erg} \mathrm{s}^{-1}$ is $\sim 190$ of which $\sim 55$ are high mass and $\sim 135$ are low mass binaries. Extrapolating the luminosity functions towards low luminosities we estimate the total number of the X-ray binaries brighter than $10^{34} \mathrm{erg} \mathrm{s}^{-1}$ as $\sim 705(\sim 325 \mathrm{LMXB}$ and $\sim 380$ HMXB sources). These estimates might be subject to the uncertainty of a factor of $\sim 2$ due to insufficient knowledge of the spatial distribution of X-ray binaries in the Galaxy.

- Due to the shallow slope of the luminosity function, the integrated X-ray emission of the Milky Way is dominated by $\sim 5-10$ brightest sources. Variability of individual sources or an outburst of a bright transient source can increase the integrated luminosity of the Milky Way by as much as a factor of $\sim 2$.

- We found that at least 16 sources in the Galaxy showed episodes of super-Eddington luminosity for a $1.4 \mathrm{M}_{\odot}$ neutron star. We plotted the distribution of these sources across the Galaxy in various projections, which can be used to compare with the recent CHANDRA and XMM-Newton images of the nearby galaxies.

The above mentioned results for the X-ray binary population facilitate the study of the properties of X-ray binary populations in other galaxies. Based on CHANDRA and ASCA observations of nearby star forming galaxies and RXTE/ASM, ASCA, and MIRKVANT/TTM data on our Galaxy and the Magellanic Clouds we studied the relation between star formation and the population of high mass X-ray binaries. The data for our Milky Way are an important calibration point for the investigation of the connection between X-ray binaries and star formation. Within the accuracy and completeness of the data available at present, we conclude that:

- The data are broadly consistent with the assumption that in a wide range of star formation rates the luminosity distribution of HMXBs in a galaxy can be approximately described by a universal luminosity function, whose normalisation is proportional to the SFR (Fig. 5.1, 6.2, 6.3). Although the accuracy of this approximation is yet to 
be determined based on a larger galaxy sample and deeper observations, we conclude from the rather limited sample available, that it might be of the order of $~ 50$ per cent or better.

In differential form the universal luminosity function can be approximated as a power law with a cut-off at $L_{c} \sim 2 \cdot 10^{40} \mathrm{erg} \mathrm{s}^{-1}$ :

$$
\frac{d N}{d L_{38}}=\left(3.3_{-0.8}^{+1.1}\right) \cdot S F R \cdot L_{38}^{-1.61 \pm 0.12} \text { for } L<L_{c}
$$

where SFR is measured in units of $\mathrm{M}_{\odot} \mathrm{yr}^{-1}$ and is the SFR for stars with $M>8$ $\mathrm{M}_{\odot}$, and $L_{38}=L / 10^{38} \mathrm{erg} \mathrm{s}^{-1}$. In cumulative form it is correspondingly:

$$
N\left(>L_{38}\right)=\left(5.4_{-1.7}^{+2.1}\right) \cdot S F R \cdot\left(L_{38}^{-0.61 \pm 0.12}-210^{-0.61 \pm 0.12}\right) .
$$

Although more subtle effects can not presently be excluded (and are likely to exist), we did not find strong non-linear dependences of the HMXB luminosity function on SFR. We also found no strong dependences of the HMXB luminosity function on other parameters of the host galaxy, such as metalicity or star formation trigger.

- Both the number and total luminosity of HMXBs in a galaxy are directly related to the star formation rate and can both be used as an independent SFR indicator.

- The total number of HMXBs is directly proportional to SFR (Fig. 6.1):

$$
\mathrm{SFR}\left[\mathrm{M}_{\odot} \mathrm{yr}^{-1}\right]=\frac{N\left(L>2 \cdot 10^{38} \mathrm{erg} \mathrm{s}^{-1}\right)}{2.9} .
$$

- The dependence of the total X-ray luminosity of a galaxy due to HMXBs on SFR has a break at $\mathrm{SFR} \approx 4.5 \mathrm{M}_{\odot} \mathrm{yr}^{-1}$.

At sufficiently high values of star formation rate, $\mathrm{SFR} \gtrsim 4.5 \mathrm{M}_{\odot} \mathrm{yr}^{-1}\left(L_{2-10 \mathrm{keV}} \gtrsim 3\right.$. $10^{40} \mathrm{erg} \mathrm{s}^{-1}$ correspondingly) the X-ray luminosity of a galaxy due to HMXBs is directly proportional to the SFR (Fig.6.5):

$$
\mathrm{SFR}\left[\mathrm{M}_{\odot} \mathrm{yr}^{-1}\right]=\frac{L_{2-10 \mathrm{keV}}}{6.7 \cdot 10^{39} \mathrm{ergs}^{-1}}
$$

At lower values of the star formation rate, $\mathrm{SFR} \lesssim 4.5 \mathrm{M}_{\odot} \mathrm{yr}^{-1}\left(L_{2-10 \mathrm{keV}} \lesssim 3 \cdot 10^{40}\right.$ erg s${ }^{-1}$ ), the $L_{X}-S F R$ relation is non-linear: (Fig.6.5):

$$
\mathrm{SFR}\left[\mathrm{M}_{\odot \mathrm{yr}^{-1}}\right]=\left(\frac{L_{2-10 \mathrm{keV}}}{2.6 \cdot 10^{39} \mathrm{ergs}^{-1}}\right)^{0.6}
$$

The non-linear $L_{X}-S F R$ dependence in the low SFR limit is not related to nonlinear SFR dependent effects in the population of HMXB sources. It is rather caused by the non-Gaussianity of the probability distribution of the integrated luminosity of a population of discrete sources. A detailed and rigorous treatment has been given in chapter 6.8 . 
- Based on CHANDRA observations of the Hubble Deep Field North we showed, that the relation Eq.(7.4) between the SFR and the X-ray luminosity of a galaxy due to HMXBs holds for distant star forming galaxies with redshifts as high as $z=1.2$ (Fig. $6.5)$.

- The good agreement of high redshift observations with theoretical predictions and the fact that X-ray observations rely exclusively on the binary nature of the sources is evidence that not only the amount of star formation at redshifts up to $\sim 1$ can be easily obtained from the above relations but also that the HMXB formation scenario is very similar at least up to this redshift.

- The entire existence of the linear regime in the $L_{X}-\mathrm{SFR}$ relation is a direct consequence of the existence of a cut-off in the luminosity function. The position of the break in the $L_{X}-S F R$ relation depends on the cut-off luminosity $L_{c}$ in the luminosity function of HMXBs as $\mathrm{SFR}_{\text {break }} \propto L_{c}^{\alpha-1}$, where $\alpha$ is the differential slope of the luminosity function. Combined with the slope of the $L_{X}-\mathrm{SFR}$ relation in the low SFR regime (Eq.(6.8)) this opens a possibility to constrain the parameters of the luminosity function studying the $L_{X}$-SFR relation alone, without actually constructing the luminosity functions, e.g. in distant unresolved galaxies.

Agreement of the predicted $L_{X}-S F R$ relation with the data both in high and low SFR regime (Fig.6.5) gives independent evidence of the existence of a cut-off in the luminosity function of HMXBs at $L_{c} \sim$ several $\times 10^{40} \mathrm{erg} \mathrm{s}^{-1}$. It also indicates that $L_{X}-S F R$ data, including those from high redshift galaxies in the Hubble Deep Field North, are consistent with the HMXB luminosity function parameters, derived from significantly fewer galaxies, than plotted in Fig.6.5.

In general, data from the Milky Way facilitate the consistent interpretation of the observations that are now available from CHANDRA, XMM-Newton, and will be in the future from other spacecraft. Only for the Milky Way have we access to data that allow us to accurately tie X-ray binaries to various properties of their surroundings or to the Galaxy in general. Thus for the foreseeable future data on our Galaxy will be the crucial calibration point in any relation we obtain for X-ray binaries in other galaxies. 


\section{Appendix}

Table 8.1.: List of all galactic sources observed with ASM

\begin{tabular}{|c|c|c|c|c|c|}
\hline Source & $\begin{array}{l}\text { source } \\
\text { type }\end{array}$ & $\begin{array}{l}\text { avg. flux } \\
\text { [cts/s] }\end{array}$ & dist. [kpc] & $\begin{array}{l}\text { opt. comp. } \\
\mathrm{V}^{*}\end{array}$ & $\begin{array}{l}\text { ref. for } \\
\text { dist. }\end{array}$ \\
\hline 1ES $1113+43.2$ & $\mathrm{CV}$ & 0.012 & 0.088 & AR UMa & (1) \\
\hline II Peg & $\mathrm{RS} C \mathrm{~V}$ & 0.148 & 0.042 & & $(2)$ \\
\hline $4 \mathrm{U} 2206+54$ & HMXB & 0.136 & 2.5 & & $(3)$ \\
\hline AB Dor & rot. var. & 0.065 & $0.02-0.03$ & & (4) \\
\hline Algol & ecl. bin. & 0.486 & 0.031 & beta 074Per & $(5)$ \\
\hline $4 \mathrm{U} 1813+50$ & $\mathrm{CV}$ & 0.213 & 0.074 & AM Her & $(6)$ \\
\hline Aql X-1 & LMXB & 2.694 & $2.2-2.4$ & V1333 Aql & $(7)$ \\
\hline AX J1820.5-1434 & pulsar & 0.971 & $4.7-11.7$ & & (8) \\
\hline $4 \mathrm{U} 0541+60$ & $\mathrm{CV}$ & 0.123 & 0.1 ass. & BY Cam & (9) \\
\hline Cas A & SNR & 4.898 & $3.4+0.3-0.1$ & & (10) \\
\hline Cen X-3 & HMXB & 4.898 & $\begin{array}{c}8 \\
10\end{array}$ & V779 Cen & $\begin{array}{l}(11) \\
(12)\end{array}$ \\
\hline Cen X-4 & LMXB & 0.087 & $1.2-2.3$ & V822 Cen & (13) \\
\hline Cep A east & mol. cl. & -0.002 & 0.725 ass. & & (14) \\
\hline Cep X-4 & HMXB & 0.11 & $3.8+-0.6$ & & (15) \\
\hline Cir X-1 & LMXB & 102.23 & 5.5 & & (16) \\
\hline Crab & SNR & 75.266 & $1.5-2.3$ & CM Tau & (17) \\
\hline CTB 33 & SNR & 0.354 & $7.8-11.3$ & & (18) \\
\hline Cyg X-1 & HMXB & 30.255 & $\begin{array}{c}2.1+-0.1 \\
1.8\end{array}$ & HD 226868 & $\begin{array}{l}(19) \\
(20)\end{array}$ \\
\hline Cyg X-2 & LMXB & 37.922 & $\begin{array}{c}7.2+-1.1 \\
8.7+-2 \\
11.6+-0.3\end{array}$ & V1341 Cyg & $\begin{array}{l}(21) \\
(22) \\
(23)\end{array}$ \\
\hline Cyg X-3 & HMXB & 12.259 & $\begin{array}{l}8.5 \text { ass. } \\
10 \text { ass. }\end{array}$ & & $\begin{array}{l}(24) \\
(25)\end{array}$ \\
\hline DM U Ma & RSCV & 0.082 & 0.13 & & $(26)$ \\
\hline Do $\operatorname{Ar} 21$ & variable & 0.195 & 0.125 & & $(27)$ \\
\hline 1E 1024.0-5732 & HMXB & 0.04 & 3 & & $(28)$ \\
\hline 2E 1048.1-5937 & HMXB & 0.055 & 3 (fit) & & $(29)$ \\
\hline 1E 1740.7-2942 & LMXB & 1.817 & $8.5(\mathrm{GC})$ & & $(30)$ \\
\hline $2 \mathrm{E} 2259.0+5836$ & LMXB & 0.897 & 4 ass. & & $(31)$ \\
\hline
\end{tabular}


table 8.1 (continued)

\begin{tabular}{|c|c|c|c|c|c|}
\hline & & & $3.6-5.5$ & & $(32)$ \\
\hline EF Eri & $\mathrm{CV}$ & 0.058 & $0.8-0.165$ & & (33) \\
\hline eta Car & variable & 0.428 & 2.3 & & $(34)$ \\
\hline EX Hya & $\mathrm{CV}$ & 0.416 & 0.1 ass. & & $(35)$ \\
\hline EXO 0748-676 & XRB & 0.576 & 10 ass. & UY Vol & $(36)$ \\
\hline EXO 1846-031 & LMXB & 0.19 & 7 & & $(37)$ \\
\hline EXO $2030+375$ & HMXB & 0.308 & 5.3 & & (38) \\
\hline$\gamma \mathrm{Cas}$ & HMXB & 0.520 & 0.188 ass. & & (39) \\
\hline GCX-1 & LMXB & 2.177 & $8.5(\mathrm{GC})$ & & $(40)$ \\
\hline N Per 01 & CV & 0.262 & 0.47 & GK Per & $(41)$ \\
\hline GRO J0422+32 & LMXB & 0.08 & 2.6 & V518 Per & $(42)$ \\
\hline & & & 3.6 & & $(37)$ \\
\hline GRO J1008-57 & HMXB & 0.093 & 5 ass. & & (43) \\
\hline GRO J1744-28 & LMXB & 5.695 & 8.5 & & $(44)$ \\
\hline GRO J2058+42 & HMXB & 0.174 & $7-16$ & & $(45)$ \\
\hline GRANAT 0834-429 & HMXB & 0.043 & $>4.5$ & & $(46)$ \\
\hline GRANAT 1737-31 & $\mathrm{XRB}$ & 0.504 & 8.5 ass. & & $(47)$ \\
\hline GRANAT 1739-278 & LMXB & 10.765 & $6-8.5$ & & $(48)$ \\
\hline GRANAT 1758-258 & LMXB & 2.134 & 10 ass. & & $(49)$ \\
\hline & & & 8.5 ass. & & $(50)$ \\
\hline RX J1829.4-2347 & LMXB & 2.126 & $\begin{array}{c}<7.4+-0.7 \\
4-22\end{array}$ & & $\begin{array}{l}(51) \\
(52)\end{array}$ \\
\hline GRANAT $1915+105$ & HMXB & 63.906 & $\begin{array}{c}12.5+-1.5 \\
7.9-14\end{array}$ & & $\begin{array}{l}(53) \\
(54)\end{array}$ \\
\hline GS $1843+009$ & HMXB & 0.272 & 10 ass. & & $(55)$ \\
\hline GINGA $2000+25$ & LMXB & 0.053 & 2.7 & QZ Vul & (37) \\
\hline GINGA $2023+338$ & LMXB & 0.082 & 3 & V404 Cyg & (37) \\
\hline GX $1+4$ & LMXB & 1.456 & 10 ass. & V2116 Oph & $(56)$ \\
\hline GX $13+1$ & LMXB & 23.018 & $\begin{array}{c}7+-1 \\
5.4-7.6\end{array}$ & & $\begin{array}{l}(57) \\
(58)\end{array}$ \\
\hline GX $17+2$ & LMXB & 46.059 & $\begin{array}{c}5-20 \\
9\end{array}$ & NP Ser & $\begin{array}{l}(59) \\
(60)\end{array}$ \\
\hline GX $3+1$ & LMXB & 19.840 & $\begin{array}{c}5.3-14.7 \\
8 \text { ass. }\end{array}$ & V3893 Sgr & $\begin{array}{l}(61) \\
(62)\end{array}$ \\
\hline GX 301-2 & HMXB & 1.985 & 1.8 & $\begin{array}{c}\text { BP Cru } \\
\text { Wray } 977\end{array}$ & (63) \\
\hline GX 304-1 & HMXB & 0.071 & 2.4 & V850 Cen & (64) \\
\hline GX 339-4 & LMXB & 5.847 & $\begin{array}{c}1.3 \\
4+-1\end{array}$ & V821 Ara & $\begin{array}{l}(65) \\
(66)\end{array}$ \\
\hline GX $340+0$ & LMXB & 30.387 & $7-14$ & & (61) \\
\hline GX $349+2$ & LMXB & 51.576 & $\begin{array}{c}8.5(\mathrm{GC}) \\
10\end{array}$ & & $\begin{array}{l}(67) \\
(68)\end{array}$ \\
\hline
\end{tabular}


table 8.1 (continued)

\begin{tabular}{|c|c|c|c|c|c|}
\hline GX 354-0 & LMXB & 6.669 & 8.5 ass. & \multirow{6}{*}{ V2216 Oph } & $(69)$ \\
\hline GX 5-1 & LMXB & 71.437 & $7-16$ & & (61) \\
\hline GX 9+1 & LMXB & 39.279 & $7-7.2$ & & (61) \\
\hline \multirow[t]{3}{*}{ GX $9+9$} & LMXB & 16.946 & 7 & & $(70)$ \\
\hline & & & 1.1-4 & & (61) \\
\hline & & & 4.4 & & $(71)$ \\
\hline HD 101379 & RSCV & 0.114 & 0.16 & & $(26)$ \\
\hline $4 \mathrm{U} 1700+24$ & LMXB & 0.208 & $0.73+-0.9$ & V934 Her & $(72)$ \\
\hline HD 245770 & HMXB & 0.133 & 2 & V725 Tau & $(73)$ \\
\hline HD 283447 & T Tauri & 0.026 & $0.15+-0.2$ & & (74) \\
\hline HD 283572 & T Tauri & 0.02 & 0.128 & & (73) \\
\hline HD 8357 & $\mathrm{RSCV}$ & 0.089 & 0.039 & & (75) \\
\hline Her X-1 & LMXB & 0.977 & 5 & HZ Her & (76) \\
\hline GPS 1733-304 & LMXB & 0.321 & 4.5 & & $(77)$ \\
\hline HR 1099 & RSCV & 0.21 & 0.36 & & $(26)$ \\
\hline HR 5110 & $\mathrm{RSCV}$ & 0.088 & $0.0445+-0.0012$ & & $(78)$ \\
\hline IC 348 & open cl. & 0.018 & 0.32 & & (79) \\
\hline IC 443 & SNR & 0.473 & 1.5 & & $(80)$ \\
\hline KS 1731-260 & LMXB & 10.473 & 8.3 ass. & & $(81)$ \\
\hline & & & 5 (uncert) & & $(37)$ \\
\hline LS I 61303 & HMXB & 0.127 & 2.3 & V615 Cas & $(82)$ \\
\hline LS IV -1459 & Star & 0.369 & 0.49 & & $(83)$ \\
\hline M 15 & LMXB & 0.966 & 10.5 & & $(84)$ \\
\hline $4 \mathrm{U} 0513-40$ & LMXB & 0.372 & 12.2 & & $(85)$ \\
\hline NGC 6440 & LMXB & 0.380 & 8.5 & & (63) \\
\hline NGC 6441 & LMXB & 2.099 & $7.4-10.2$ & & $(86)$ \\
\hline NGC 6624 & LMXB & 19.828 & $6.4+-0.6$ & & $(87)$ \\
\hline & & & 8 & & (88) \\
\hline NGC 6652 & LMXB & 0.718 & 14.3 & & $(89)$ \\
\hline NGC 6712 & LMXB & 0.583 & 6.8 & & $(85)$ \\
\hline N Cas 95 & Nova & 0.037 & 3.9 & & $(90)$ \\
\hline NGC 1333 & refl. neb. & 0.009 & 0.5 & & (91) \\
\hline NGC 2024 & SNR & 0.088 & 0.415 & & $(92)$ \\
\hline N Musc 91 & LMXB & 0.07 & 5.5 & GU Mus & (93) \\
\hline N Oph 77 & LMXB & 0.352 & 4.3 & V2107 Oph & (93) \\
\hline N Oph 93 & LMXB & 0.296 & 2.4 & V2293 Oph & (93) \\
\hline N Sco 94 & LMXB & 49.471 & 3.2 & V1033 Sco & (94) \\
\hline Tra X-1 & LMXB & 0.245 & 4.4 & KY TrA & $(95)$ \\
\hline N Vela 93 & LMXB & 0.031 & 3 & MM Vel & (93) \\
\hline PSR 0021-72j & pulsar & -0.067 & $4.1-4.9$ & & $(96)$ \\
\hline PSR 1259-63 & pulsar & 0.177 & 4.6 & & $(96)$ \\
\hline PSR 1744-24a & pulsar & 0.546 & 3.9 & & $(96)$ \\
\hline
\end{tabular}


table 8.1 (continued)

\begin{tabular}{|c|c|c|c|c|c|}
\hline PSR $1908+00$ & pulsar & -0.132 & $3.7-4.5$ & & $(96)$ \\
\hline PSR $1957+20$ & pulsar & 0.06 & 1.5 & & (96) \\
\hline PSR J0437-4715 & pulsar & 0.132 & 0.14 & & (96) \\
\hline PSR J0700+6418 & pulsar & 0.05 & 1.4 & & (97) \\
\hline PSR J0751+1807 & pulsar & 0.059 & 2 & & (98) \\
\hline PSR J1012+5307 & pulsar & -0.035 & 0.52 & & (99) \\
\hline PSR J1022+1001 & pulsar & 0.02 & 0.6 & & $(100)$ \\
\hline PSR J1713+0747 & pulsar & 0.074 & $1.1+0.5-0.3$ & & (101) \\
\hline PSR J2051-0827 & pulsar & 0.021 & 1.3 & & (102) \\
\hline PSR J2145-0750 & pulsar & 0.059 & 0.5 & & (103) \\
\hline Puppis A & SNR & 0.835 & $2.2+-0.3$ & & (104) \\
\hline RX J0146.9+6121 & HMXB & 0.124 & $0.7+-0.3$ & LS I +61 235 & (105) \\
\hline RX J0812.4-312 & HMXB & 0.117 & 9 & & (106) \\
\hline RX J0925.7-4758 & $\mathrm{CV}$ & 0.047 & $>10$ & MR Vel & (107) \\
\hline RX J1037.5-568 & HMXB & 0.067 & 5 & & (108) \\
\hline XTE J1709-267 & XRB & 0.61 & $10+-1$ & & (109) \\
\hline QR And & variable & -0.108 & 2.38 & QR And & (110) \\
\hline SAO 085590 & variable & 0.018 & 0.05 & & (111) \\
\hline SAO 108231 & $\mathrm{RSCV}$ & 0.169 & $0.0968+-0.0071$ & & $(78)$ \\
\hline SAX J1747.0-2853 & LMXB & 4.257 & $<8$ & & (112) \\
\hline SAX J1750-29 & $\mathrm{XRB}$ & 1.159 & 7 & & (113) \\
\hline SAX J1808.4-3658 & LMXB & 0.132 & 4.4 & & (114) \\
\hline SAX J2103.5+4545 & pulsar & 0.157 & 4 & & (115) \\
\hline Sco X-1 & LMXB & 933.72 & $\begin{array}{c}2.8+-0.3 \\
2\end{array}$ & V818 Sco & $\begin{array}{l}(116) \\
(117)\end{array}$ \\
\hline Sct X-1 & HMXB & 0.196 & $10+-4$ & & (118) \\
\hline Ser X-1 & LMXB & 16.579 & $\begin{array}{c}7 \\
5 \text { ass. }\end{array}$ & MM Ser & $\begin{array}{l}(71) \\
(119)\end{array}$ \\
\hline Sgr 1806-20 & SNR & 0.433 & 14.5 & & (120) \\
\hline Sgr $1900+14$ & SNR & 0.227 & 10 ass. & & (121) \\
\hline$\sigma$ Gem & RSCV & 0.158 & 0.059 & & $(26)$ \\
\hline SLX 1735-269 & LMXB & 1.272 & 8.5 ass. & & $(122)$ \\
\hline SS 0019+21 & $\mathrm{CV}$ & 0.05 & 2 & & (123) \\
\hline SS 433 & HMXB & 0.518 & 5.5 & V1343 Aql & (124) \\
\hline SS Cyg & $\mathrm{CV}$ & 0.333 & $0.166+-0.127$ & & (125) \\
\hline Tycho & SNR & 1.29 & $2.2-4.5$ & & (126) \\
\hline U Gem & $\mathrm{CV}$ & 0.108 & $0.0964+-0.0046$ & & $(125)$ \\
\hline UX Ari & RSCV & 0.162 & 0.05 & & (127) \\
\hline V $0332+53$ & HMXB & 0.048 & 7 & BQ Cam & $(38)$ \\
\hline V1223 Sgr & $\mathrm{CV}$ & 0.539 & 0.4 & & (128) \\
\hline V4641 Sgr & LMXB & 0.774 & 0.5 & & (129) \\
\hline Vela & SNR & 0.756 & 0.5 & & (130) \\
\hline
\end{tabular}


table 8.1 (continued)

\begin{tabular}{|c|c|c|c|c|c|}
\hline & & & $0.285-0.12$ & & (131) \\
\hline Vela X-1 & HMXB & 4.267 & $1.9+-0.2$ & GP Vel & (132) \\
\hline & & & 1 & & (133) \\
\hline & & & $1.4+-0.5$ & & (134) \\
\hline VW Hyi & $\mathrm{CV}$ & 0.026 & 0.065 & & (135) \\
\hline $\mathrm{X} 0042+327$ & LMXB & 0.042 & 7 & & (93) \\
\hline X $0114+650$ & HMXB & 0.248 & 7 & V662 Cas & (136) \\
\hline \multirow[t]{4}{*}{$\mathrm{X} 0115+634$} & HMXB & 0.521 & 4 & V635 Cas & (137) \\
\hline & & & 2.5 & & (138) \\
\hline & & & 4.4 & & (139) \\
\hline & & & 6 & & $(38)$ \\
\hline X $0142+614$ & LMXB & 0.390 & 1 & & (140) \\
\hline X $0614+091$ & LMXB & 3.567 & $<3$ & V1055 Ori & (141) \\
\hline X 0620-003 & LMXB & 0.047 & 0.87 & V616 Mon & (93) \\
\hline \multirow[t]{2}{*}{ X 0726-260 } & HMXB & 0.102 & $4.6+-1.3$ & LS 437 & (142) \\
\hline & & & $6.1+-0.3$ & & (143) \\
\hline X 0836-429 & LMXB & 0.035 & 10 & & (93) \\
\hline X 0918-548 & LMXB & 0.542 & 15 & & (144) \\
\hline \multirow[t]{2}{*}{ X 1118-616 } & HMXB & 0.086 & 4 & Hen 3-640 & (145) \\
\hline & & & 6 & & $(38)$ \\
\hline X 1145-616 & HMXB & 0.415 & 8 & V830 Cen & (146) \\
\hline \multirow[t]{3}{*}{ X 1145-619 } & HMXB & 0.211 & $0.52+-0.24$ & V801 Cen & (147) \\
\hline & & & 3.1 & & $(38)$ \\
\hline & & & 1.5 & & (148) \\
\hline X 1254-690 & LMXB & 2.403 & $8-15$ & GR Mus & (149) \\
\hline X 1323-619 & LMXB & 0.497 & 10 ass. & & (150) \\
\hline X 1417-624 & HMXB & 0.158 & 6 & & $(38)$ \\
\hline X 1538-522 & HMXB & 0.917 & $6.4+-1$ & QV Nor & (151) \\
\hline X 1543-475 & LMXB & 0.031 & $9.1+-1.1$ & & (152) \\
\hline X 1543-624 & LMXB & 2.828 & 10 ass. & & (153) \\
\hline X 1553-542 & HMXB & 0.106 & 10 & & (154) \\
\hline X 1556-605 & LMXB & 1.546 & 10 & & (61) \\
\hline X 1608-522 & LMXB & 3.631 & 3.6 & QX Nor & (155) \\
\hline X 1624-490 & LMXB & 3.634 & $7-20$ & & (61) \\
\hline X 1627-673 & LMXB & 0.669 & 8 & & (156) \\
\hline X 1630-472 & LMXB & 3.763 & $>4$ & & (157) \\
\hline & & & 10 & & (158) \\
\hline X 1636-536 & LMXB & 14.675 & 5.9 & V801 Ara & (159) \\
\hline & & & $0.7-6.5$ & & (61) \\
\hline X 1657-415 & HMXB & 0.973 & $>11$ & & (160) \\
\hline X 1658-298 & LMXB & 0.4 & 10 & & $(71)$ \\
\hline 4U 1700-377 & HMXB & 4.423 & $>0.58$ & V884 Sco & (147) \\
\hline
\end{tabular}


table 8.1 (continued)

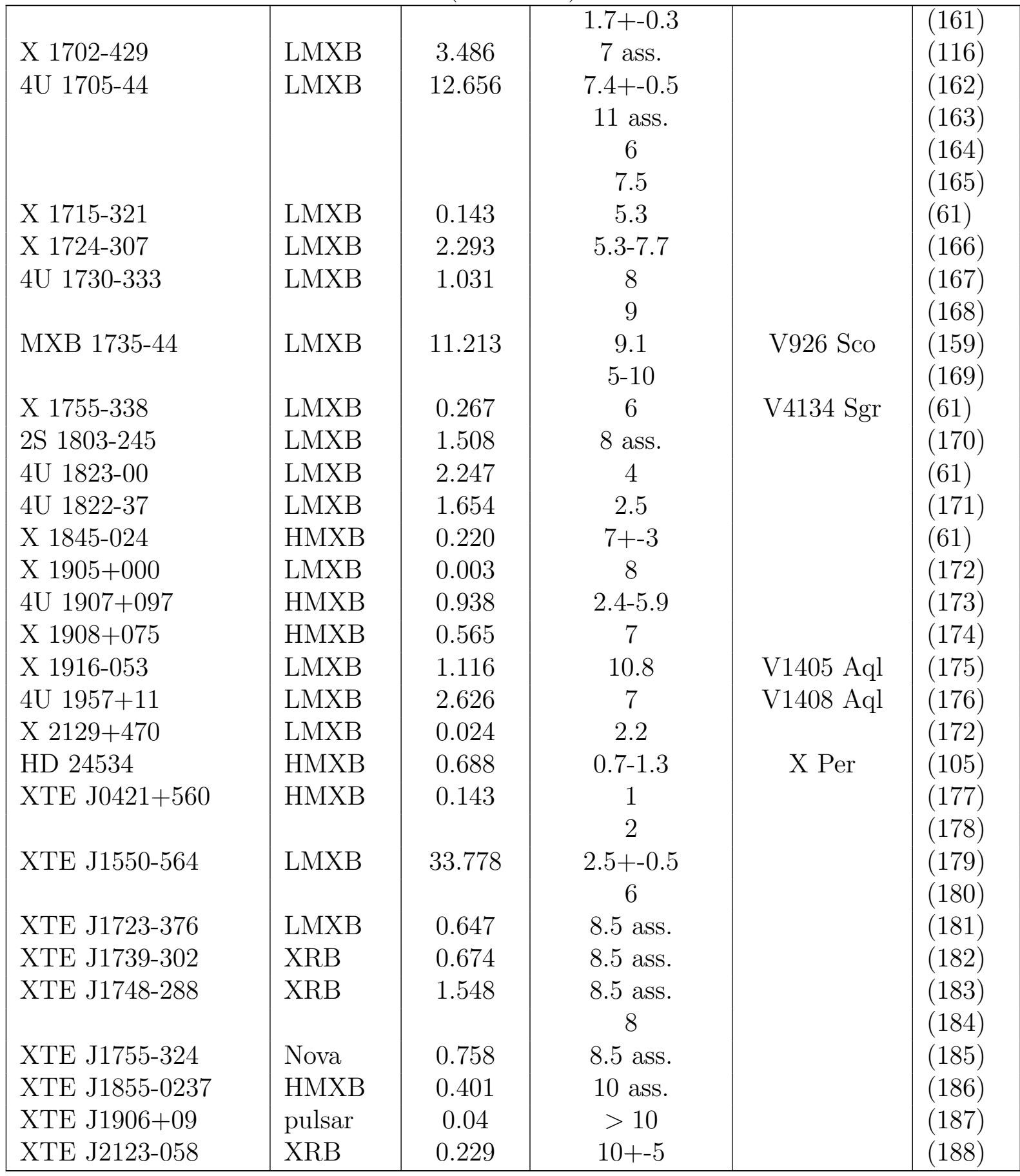

(1) Remillard et al. ApJ 426, 288, 94

(2) Kashyap and Drake ApJ 524, 988, 99

(3) Saraswat and Apparao ApJ 401, 678, 92
(95) Barret et al. A\&A 296, 459, 95

(96) Taylor, Manchester and Lyne ApJS 88, 529,93

(97) van Kerkwijk and Kulkarni ApJL 454, 141,95 
(4) Rucinski et al. ApJ 449, 900, 95

(5) Ottmann and Schmitt A\&A 307, 813, 96

(6) Bradford et al. ApJ 513, 491, 99

(7) Shabaz et al. MNRAS 300, 103598

(8) Kinugasa et al. ApJ 495, 435, 98

(9) Kallmann et al. ApJ 466, 973, 96

(10) Reed et al. ApJ 440, 706, 95

(11) Krzeminski ApJL 192, 135, 74

(12) Hutchings et al. ApJ 229, 1079, 79

(13) Rutledge et al. ApJ 514, 945, 99

(14) Hughes ApJ 383, 280, 91

(15) Wilson, Finger and Scott ApJ 511, 367, 99

(16) Gierlinski et al. MNRAS 309, 469, 99

(17) Wallace et al. ApJS 124, 181, 99

(18) Sarma et al. ApJ 483, 335, 97

(19) Massey, Johnson and De Gioia-Eastwood ApJ 454, 151, 95

(20) Malysheva AL 23, 585, 97

(21) Orosz and Kuulkers MNRAS 305, 132, 99

(22) Cowley, Crampton and Hutchings ApJ 231, 539, 79

(23) Smale ApJL 498, 141, 98

(24) Fender, Hanson and Pooley MNRAS 308, 473,99

(25) Schalinski et al. A\&A 329, 504, 98

(26) Mitrou et al. A\&A 317, 776, 97

(27) Gomez, Whitney and Wood AJ 115, 2018, 98

(28) Reig A\&A 345, 576, 99

(29) Oosterbroek et al. A\&A 334, 925, 98

(30) Kuznetsov et al. MNRAS 292, 651, 97

(31) Parmar et al. A\&A 330, 175, 98

(32) Rho and Petre ApJ 484, 828, 97

(33) Beuermann A\&A 354, L49, 00

(34) van Genderen et al. A\&A 343, 847, 98

(35) Mauche ApJ 520, 822, 99

(36) Church et al. ApJ 504, 516, 98

(37) Barret, McClintock and Grindlay ApJ $473,963,96$

(38) Negueruela A\&A 338, 505, 98

(39) Owens et al. A\&A 348, 170, 99
(98) Becker et al. MNRAS 282, L33, 96

(99) Halpern ApJL 459, 9, 96

(100) Kramer et al. ApJ 520, 324, 99

(101) Camilo, Foster and Wolszczan ApJL 437, 39, 94

(102) Stappers et al. ApJL 510, 45, 99

(103) Bell et al. ApJL 452, 121, 95

(104) Pavlov, Zavlin and Truemper ApJL 511, 45,99

(105) Haberl et al. A\&A 330, 189, 98

(106) Reig and Roche MNRAS 306, 95, 99

(107) Motch A\&A 338, L13, 98

(108) Reig and Roche MNRAS 306, 100, 99

(109) Cocchi et al. ApJ 508, 163, 98

(110) Meyer-Hofmeister et al. A\&A 331, 612, 98

(111) Leroy A\&AS 101, 551, 93

(112) Campana, Israel and Stella IAUC 7401, 1,00

(113) Natalucci et al. ApJL 523, 45, 99

(114) Ergma and Antipova A\&A 343, L45, 99

(115) Hulleman, in 't Zand, and Heise A\&A 337, L25, 98

(116) Markwardt, Strohmayer and Swank ApJL 512, 125, 99

(117) Kallman, Boroson and Vrtilek ApJ 502, 441, 98

(118) Koyama et al. Nature 343, 148, 90

(119) Balucinska and Czerny AcA 35, 291, 85

(120) Fuchs et al. A\&A 350, 891, 99

(121) Murakami et al. ApJL 510, 119, 99

(122) Wijnands and van der Klis A\&A 345, L35, 99

(123) Becker et al. ApJ 506, 880, 98

(124) Safi-Harb and Petre ApJ 512, 784, 99

(125) Harrison et al. ApJL 515, 93, 99

(126) Reynoso et al. AJ 117, 1827, 99

(127) Franciosini et al. A\&A 341, 595, 99

(128) Patterson PASP 106, 209, 94

(129) in 't Zand et al. A\&A 357, 520, 00

(130) Oegelman, Koch-Miramond and Auriére ApJ 342, L83, 89

(131) Zimmermann, Trümper and Yorke (eds.) MPE Report 263, 173, 96

(132) Sadakane et al. ApJ 288, 284, 85

(133) Hiltner, Werner and Osmer ApJL 175, 19,72 
(40) Churazov et al. ApJ 443, 341, 95

(41) Balman and Ögelman ApJL 518, 111, 99

(42) Menou et al. ApJ 520, 276, 99

(43) Shrader et al. ApJ 512, 920, 99

(44) Nishiuchi et al. ApJ 517, 436, 99

(45) Wilson et al. ApJ 499, 820, 98

(46) Wilson et al. 479, 388, 97

(47) Cui et al. ApJL 487, 73, 97

(48) Greiner, Dennerl and Predehl A\&AL 314, 21, 96

(49) Mereghetti et al. ApJ 476, 829, 97

(50) Marti et al. A\&AL 338, 95, 98

(51) in't Zand et al. A\&A 347, 891, 99

(52) Barret, Motch and Pietsch A\&A 303, 526, 95

(53) Mirabel and Rodriguez Nature 371, 46, 94

(54) Chaty, et al. A\&A 310, 825, 96

(55) Manchanda MNRAS 305, 409, 99

(56) Kotani et al. ApJ 510, 369, 99

(57) Bandyopadhyay et al. MNRAS 306, 417, 99

(58) Garcia et al. AJ 103, 1325, 92

(59) Deutsch et al. ApJ 524, 406, 99

(60) Penninx, van Paradijs and Zijlstra A\&A 240, 317, 90

(61) Christian and Swank ApJS 109, 177, 97

(62) Asai et al. PASJ 45, 801, 93

(63) Parkes et al. MNRAS 191, 547, 80

(64) Pietsch et al. A\&A 163, 93, 86

(65) Predehl, Braeuninger and Schmitt A\&A 246, L40, 91

(66) Zdziarski et al. MNRAS 301, 435, 98

(67) Campos-Aguilar and Moles A\&A 241, 358,91

(68) van Paradijs and McClintock A\&A 290, 133,94

(69) Asai et al. PASJ 46, 479, 94

(70) Schaefer ApJ 354, 720, 90

(71) Smith, Beall and Swain AJ 99, 273, 90

(72) Garcia et al. ApJ 267, 291, 83

(73) Favata, Micela and Sciortino A\&A 337, 413,98
(134) Chevalier and Ilovaisky A\&A 330, 201, 98

(135) Hartmann et al. A\&A 349, 588, 99

(136) Liu and Hang A\&A 350, 855, 99

(137) Tamura et al. ApJ 389, 676, 92

(138) Rappaport et al. ApJL 224, 1, 78

(139) Ricketts et al. SSR 30, 399, 81

(140) Israel et al. A\&A 346, 929, 99

(141) Piraino et al. A\&A 349, L77, 99

(142) Negueruela et al. A\&A 315, 160, 96

(143) Corbet and Peele ApJL 489, 83, 97

(144) Chevalier and Ilovaisky A\&A 172, 167, 87

(145) Motch et al. A\&A 201, 63, 88

(146) Hutchings et al. PASP 99, 420, 87

(147) Clark and Dolan A\&A 350, 1085, 99

(148) Hammerschlag-Hensberge et al. A\&A $85,119,80$

(149) Smale and Wachter ApJ 527, 341, 99

(150) Balucinska-Church et al. A\&A 349, 495, 99

(151) Bulik et al. ApJ 444, 405, 95

(152) Orosz et al. ApJ 499, 375, 98

(153) Singh, Apparao and Kraft ApJ 421, 753, 94

(154) Kelley, Rappaport and Ayasli ApJ 274, 765,83

(155) Nakamura et al. PASJ 41, 617, 89

(156) Chakrabarty ApJ 492, 342, 98

(157) Hjellming et al. ApJ 514, 383, 99

(158) Parmar, Stella and White ApJ 304, 664, 86

(159) Augusteijn et al. A\&A 332, 561, 98

(160) Orlandini et al. A\&A 349, L9, 99

(161) Bolton and Herbst AJ 81, 339, 76

(162) Haberl and Titarchuk A\&A 299, 414, 95

(163) Ford, van der Klis and Kaaret ApJ 498, L41, 98

(164) Tavani and Liang A\&AS 120, C133, 96

(165) Barret et al. A\&AS 120, C121, 96

(166) Barret et al. A\&A 341, 789, 99

(167) Ortolani, Bica and Barbuy A\&A 306, 134,96 
(74) Phillips et al. AJ 111, 918, 96

(75) Fekel AJ 112, 269, 96

(76) Bradt and McClintock ARA\&A 21, 13, 83

(77) Ortolani, Bica and Barbuy A\&A 267, 66, 93

(78) van den Berg, Verbunt and Mathieu A\&A $347,866,99$

(79) Duchêne, Bouvier and Simon A\&A 343, 831, 99

(80) Rivinius, Stefl and Baade A\&A 348, 831, 99

(81) Barret, Motch and Predehl A\&A 329, 965,98

(82) Harrison et al. ApJ 528, 454, 00

(83) Lahulla and Hilton A\&AS 94, 265, 92

(84) Callanan, Drake and Fruscione ApJL 521, 125,99

(85) Peterson ASP Conf. Ser. 50, 337, 93

(86) Sansom et al. MNRAS 262, 429, 93

(87) Vacca, Lewin and van Paradijs MNRAS 220, 339, 86

(88) Sosin and King AJ 109, 639, 95

(89) in 't Zand et al. A\&A 329, L37, 98

(90) Kamath and Ashok A\&AS 136, 107, 99

(91) Martini, Sellgren and DePoy ApJ 526, 772,99

(92) Wiesemeyer et al. A\&A 320, 287, 97

(93) Chen, Shrader and Livio ApJ 491, 312, 97

(94) Tomsick et al. ApJ 512, 892, 99
(168) Malkan, Kleinmann and Apt ApJ 237, 432,80

(169) McClintock, Canizares and Backman ApJL 223, 75, 78

(170) Wijnands and van der Klis ApJ 522, 965, 99

(171) Clay et al. A\&A 255, 167, 92

(172) Shabaz et al. MNRAS 282, 1437,96

(173) in't Zand, Baykal and Strohmayer ApJ 496, 386, 98

(174) Wen, Remillard and Bradt ApJ 532, 1119,00

(175) Ko et al. ApJ 520, 292, 99

(176) Nowak and Wilms ApJ 522, 476, 99

(177) Bergner et al. A\&AS 112, 221, 95

(178) Clark et al. A\&A 348, 888, 99

(179) Sanchez-Fernandez et al. A\&A 348, L9, 99

(180) Remillard et al. ApJL 517, 127, 99

(181) Marshall, Ueda and Markwardt IAUC 7133, 1, 99

(182) Smith et al. ApJL 501, 181, 98

(183) Sidoli et al. ApJ 525, 215, 99

(184) Mirabel and Rodriguez ARA\&A 37, 409, 99

(185) Revnitsev et al. AL 25, 493, 99

(186) Corbet et al. ApJ 517, 956, 99

(187) Marsden et al. ApJL 502, 129, 98

(188) Gneiding, Steiner and Cieslinski A\&A 352, 543, 99 


\section{Bibliography}

Abramowicz, M., Czerny, B., Lasota, J., \& Szuszkiewicz, E. 1988, ApJ, 332, 646

Armus, L., Heckman, T. M., \& Miley, G. K. 1990, ApJ, 364, 471

Awaki, H., Matsumoto, H., \& Tomida, H. 2002, ApJ, 567, 892

Bahcall, J. 1978, in Proceedings of the International School of Physics "Enrico Fermi", Vol. 65, Physics and astrophysics of neutron stars and black holes, ed. R. Giacconi \&

R. Ruffini (Italian Phys. Soc.), 63

Bahcall, J. N. 1983, ApJ, 267, 52

Bahcall, J. N. \& Soneira, R. M. 1980, ApJS, 44, 73

Bandyopadhyay, R. M., Shahbaz, T., Charles, P. A., \& Naylor, T. 1999, MNRAS, 306, 417

Barret, D., McClintock, J. E., \& Grindlay, J. E. 1996, ApJ, 473, 963

Barret, D., Motch, C., \& Predehl, P. 1998, A\&A, 329, 965

Basko, M. M. \& Sunyaev, R. A. 1976, MNRAS, 175, 395

Bell, E. F. \& Kennicutt, R. C. 2001, ApJ, 548, 681

Bildsten, L. \& Chakrabarty, D. 2001, ApJ, 557, 292

Binney, J., Gerhard, O., \& Spergel, D. 1997, MNRAS, 288, 365

Bolton, C. T. 1972, Nature Physical Science, 240, 124+

Bondi, H. \& Hoyle, F. 1944, MNRAS, 104, 273

Bradshaw, C. F., Fomalont, E. B., \& Geldzahler, B. J. 1999, ApJ, 512, L121

Bradt, H. V., Rothschild, R. E., \& Swank, J. H. 1993, A\&AS, 97, 355

Brandt, H., Rotschild, R., \& Swank, J. 1996, in Memorie della Societa Astronomica Italiana, Vol. 67, 593

Brandt, W. N., Alexander, D. M., Hornschemeier, A. E., et al. 2001, AJ, 122, 2810 
Brinchmann, J. \& Ellis, R. S. 2000, ApJ, 536, L77

Buat, V., Boselli, A., Gavazzi, G., \& Bonfanti, C. 2002, A\&A, 383, 801

Chakrabarty, D., Grunsfeld, J. M., Prince, T. A., et al. 1993, ApJ, 403, L33

Chandrasekhar, S. 1950, Radiative transfer. (Oxford, Clarendon Press, 1950.)

Chevalier, C. \& Ilovaisky, S. A. 1990, A\&A, 238, 163

Christian, D. J. \& Swank, J. H. 1997, ApJS, 109, 177

Cohen, J. G., Hogg, D. W., Blandford, R., et al. 2000, ApJ, 538, 29

Condon, J. J. 1992, ARA\&A, 30, 575

Condon, J. J., Helou, G., Sanders, D. B., \& Soifer, B. T. 1990, ApJS, 73, 359

Corbet, R. H. D., Marshall, F. E., Peele, A. G., \& Takeshima, T. 1999, ApJ, 517, 956

Cowley, A. P., Crampton, D., \& Hutchings, J. B. 1979, ApJ, 231, 539

Dalton, W. W. \& Sarazin, C. L. 1995, ApJ, 440, 280

David, L. P., Jones, C., \& Forman, W. 1992, ApJ, 388, 82

de Vaucouleurs, G., de Vaucouleurs, A., Corwin, H. G., et al. 1991, Third Reference Catalogue of Bright Galaxies (Volume 1-3, XII, 2069 pp. 7 figs.. Springer-Verlag Berlin Heidelberg New York)

Dehnen, W. \& Binney, J. 1998, MNRAS, 294, 429

Djorgovski, S. 1993, in ASP Conf. Ser. 50: Structure and Dynamics of Globular Clusters, 373

Downes, D., Wilson, T. L., Bieging, J., \& Wink, J. 1980, A\&AS, 40, 379

Dwek, E., Arendt, R., Hauser, M., et al. 1995, ApJ, 445, 716

Ebisuzaki, T., Sugimoto, D., \& Hanawa, T. 1984, PASJ, 36, 551

Eneev, T. M., Kozlov, N. N., \& Sunyaev, R. A. 1973, A\&A, 22, 41+

Englmaier, P. \& Gerhard, O. 1999, MNRAS, 304, 512

Eracleous, M., Shields, J. C., Chartas, G., \& Moran, E. C. 2002, ApJ, 565, 108

Fabbiano, G. 1994, Cambridge Astrophysics Series, Vol. 26, X-ray binaries (Cambridge University Press), 390 
Fabbiano, G., Gioia, I. M., \& Trinchieri, G. 1988, ApJ, 324, 749

Fabrika, S. \& Mescheryakov, A. 2000, in IAU Symposium, Vol. 205, E105

Feitzinger, J. V. 1980, Space Science Reviews, 27, 35

Fender, R. P. 2001, MNRAS, 322, 31

Forman, W., Jones, C., Cominsky, L., et al. 1978, ApJS, 38, 357

Frank, J., King, A., \& Raines, D. 1992, Accretion power in astrophysics (Cambridge University Press)

Fryer, C. L. \& Kalogera, V. 2001, ApJ, 554, 548

Galletta, G. \& Recillas-Cruz, E. 1982, A\&A, 112, 361

Georgakakis, A., Forbes, D. A., \& Norris, R. P. 2000, MNRAS, 318, 124

Georgelin, Y. \& Georgelin, Y. 1976, A\&A, 49, 57

Ghosh, P. \& White, N. E. 2001, ApJ, 559, L97

Giacconi, R., Gursky, H., Paolini, F. R., \& Rossi, B. B. 1962, Physical Review Letters, 9, 439

Gnedin, I. N. \& Suniaev, R. A. 1974, A\&A, 36, 379

Gonzalez Delgado, R. M. \& Perez, E. 1996, MNRAS, 281, 1105

Grebenev, S. A., Pavlinsky, M. N., \& Sunyaev, R. 1996, in Röntgenstrahlung from the Universe, 141

Greiner, J., Cuby, J. G., McCaughrean, M. J., Castro-Tirado, A. J., \& Mennickent, R. E. 2001, A\&A, 373, L37

Greiner, J., Hasinger, G., Molendi, S., \& Ebisawa, K. 1994, A\&A, 285, 509

Griffiths, R., Ptak, A., Feigelson, E., et al. 2000, Science, 290, 1325

Griffiths, R. E. \& Padovani, P. 1990, ApJ, 360, 483

Grimm, H.-J., Gilfanov, M., \& Sunyaev, R. 2002, A\&A, 391, 923

Grimm, H.-J., M., G., \& R., S. 2003, MNRAS, 339, 793

Haberl, F. \& Titarchuk, L. 1995, A\&A, 299, 414

Hillenbrand, L. A. 1997, AJ, 113, 1733 
Holtzman, J. A., Gallagher, J. S., Cole, A. A., et al. 1999, AJ, 118, 2262

Hoopes, C. G., Walterbos, R. . M., \& Bothun, G. D. 2001, ApJ, 559, 878

Huchtmeier, W. K. \& Richter, O.-G. 1988, A\&A, 203, 237

Hutchings, J. B., Cowley, A. P., Crampton, D., van Paradus, J., \& White, N. E. 1979, ApJ, 229, 1079

Iben, I. J., Tutukov, A. V., \& Yungelson, L. R. 1995, ApJS, 100, 217

Icko Iben, j., Tutkov, A. V., \& Yungelson, L. R. 1995, ApJS, 100, 233

Illarionov, A. \& Sunyaev, R. 1975, A\&A, 39, 185

Israel, F. P. 1988, A\&A, 194, 24

Israel, G., Negueruela, I., Campana, S., et al. 2001, A\&A, 371, 1018

Jansen, F., Lumb, D., Altieri, B., et al. 2001, A\&A, 365, L1

Körding, E., Falcke, H., \& Markoff, S. 2002, A\&A, 382, L13

Kaaret, P. 2001, ApJ, 560, 715

Kalogera, V., Narayan, R., Spergel, D. N., \& Taylor, J. H. 2001, ApJ, 556, 340

Kaper, L., Lamers, H. J. G. L. M., Ruymaekers, E., van den Heuvel, E. P. J., \& Zuidervijk, E. J. 1995, A\&A, 300, 446

Karachentsev, I., Drozdovsky, I., Kajsin, S., et al. 1997, A\&AS, 124, 559

Kennicutt, R. C. 1998, ARA\&A, 36, 189

Kennicutt, R. C., Tamblyn, P., \& Congdon, C. E. 1994, ApJ, 435, 22

Kilgard, R. E., Kaaret, P. E., Krauss, M. I., et al. 2001, American Astronomical Society Meeting

King, A., Davies, M., Ward, M., Fabbiano, G., \& Elvis, M. 2001, ApJ, 552, L109

King, A. R. 1993, MNRAS, 260, L5

Kitamoto, S., Tsunemi, H., Miyamoto, S., \& Hayashida, K. 1992, ApJ, 394, 609

Koerding, E., Falcke, H., Markoff, S., \& Fender, R. 2001, in Astronomische Gesellschaft Meeting Abstracts, Vol. 18, 176

Koyama, K., Kawada, M., Kunieda, H., Tawara, Y., \& Takeuchi, Y. 1990, Nature, 343, 148 
Krzeminski, W. 1974, ApJ, 192, L135

Kuijken, K. \& Rich, R. M. 2001, in American Astronomical Society Meeting, Vol. 199, 9113

Kuno, N. \& Nakai, N. 1997, PASJ, 49, 279

Lípari, S., Díaz, R., Taniguchi, Y. andTerlevich, R., Dottori, H., \& Carranza, G. 2000, AJ, 120,645

Lamb, R. C., Markert, T. H., Hartman, R. C., Thompson, D. J., \& Bignami, G. F. 1980, ApJ, 239, 651

Lehnert, M. D. \& Heckman, T. M. 1996, ApJ, 472, 546+

Levine, A. M., Bradt, H., Cui, W., et al. 1996, ApJ, 469, L33

Lewin, W. H., van den Heuvel, E. P., \& van Paradijs, J., eds. 1994, Cambridge Astrophysics Series, Vol. 26, X-ray binaries (Cambridge University Press)

Lira, P., Ward, M., Zezas, A., Alonso-Herrero, A., \& Ueno, S. 2002, MNRAS, 330, 259

Liu, C. T. \& Kennicutt, R. C. 1995, ApJ, 450, 547+

Liu, Q. Z., van Paradijs, J., \& van den Heuvel, E. P. J. 2000, A\&AS, 147, 25

—. 2001, A\&A, 368, 1021

Lochner, J. \& Remillard, R. 1997, The XTE All Sky Monitor Data Products, http://heasarc.gsfc.nasa.gov/docs/xte/ asm_products_guide.html

Lyutyi, V. M., Syunyaev, R. A., \& Cherepashchuk, A. M. 1973, Soviet Astronomy, 17, 1+

Madau, P. \& Pozzetti, L. 2000, MNRAS, 312, L9

Maddalena, R., Morris, M., Moscowitz, J., \& Thaddeus, P. 1986, ApJ, 303, 375

Markert, T., Winkler, P., Laird, F., et al. 1979, ApJS, 39, 573

Martin, A. C., Casares, J., Charles, P. A., van der Hooft, F., \& van Paradijs, J. 1995, MNRAS, 274, L46

Martin, C. L., Kobulnicky, H. A., \& Heckman, T. M. 2002, ApJ, 574, 663

Massey, P., Johnson, K. E., \& Degioia-Eastwood, K. 1995, ApJ, 454, 151

Matilsky, T., Gursky, H., Kellogg, E., et al. 1973, ApJ, 181, 753

McKee, C. F. \& Williams, J. P. 1997, ApJ, 476, 144 
Mirabel, I. \& Rodriguez, L. 1994, Nature, 371, 46

Mirabel, I. \& Rodríguez, L. 1999, ARA\&A, 37, 409

Moshir, M., Copan, G., Conrow, T., et al. 1993, VizieR Online Data Catalog, 2156, 0

Motch, C., Haberl, F., Dennerl, K., Pakull, M., \& Janot-Pacheco, E. 1997, A\&A, 323, 853

Murdoch, H. S. \& Crawford, D. F. 1973, ApJ, 183, 1

Nagase, F. 1989, in Two Topics in X-Ray Astronomy, Volume 1: X Ray Binaries. Volume 2: AGN and the X Ray Background, 45-55

Nakamura, N., Dotani, T., Inoue, H., et al. 1989, PASJ, 41, 617

Narayan, R. \& Yi, I. 1995, ApJ, 452, 710

Negishi, T., Onaka, T., Chan, K.-W., \& Roellig, T. L. 2001, A\&A, 375, 566

Nishiuchi, M., Koyama, K., Maeda, Y., et al. 1999, ApJ, 517, 436

Ogasaka, Y., Kii, T., Ueda, Y., et al. 1998, AN, 319, 43

Oort, M. J. A. 1987, A\&AS, 71, 221

Orosz, J. 2002, in IAU Symposium: A Massive Star Odyssey, from Main Sequence to Supernova, Vol. 212

Orosz, J. A., Bailyn, C. D., McClintock, J. E., \& Remillard, R. A. 1996, ApJ, 468, 380

Orosz, J. A., Groot, P. J., van der Klis, M., et al. 2002, ApJ, 568, 845

Orosz, J. A. \& Kuulkers, E. 1999, MNRAS, 305, 132

Orosz, J. A., Kuulkers, E., van der Klis, M., et al. 2000, in American Astronomical Society Meeting, Vol. 197, 8320

Paczynsky, B. \& Wiita, P. 1980, A\&A, 88, 23

Penninx, W. 1989, in X-ray astronomy, ed. J. Hunt \& B. Battrick, Proceedings of the 23rd ESLAB Symposium No. ESA SP-296 (ESA), 185

Persic, M. \& Salucci, P. 1988, MNRAS, 234, 131

Piccinotti, G., Mushotzky, R., Boldt, E., et al. 1982, ApJ, 253, 485

Popham, R. \& Sunyaev, R. 2001, ApJ, 547, 355

Portegies Zwart, S. F. \& Yungelson, L. R. 1998, A\&A, 332, 173 
Predehl, P., Burwitz, V., Paerels, F., \& Trümper, J. 2000, A\&A, 357, L25

Prendergast, K. H. \& Burbidge, G. R. 1968, ApJ, 151, L83+

Ptak, A., Griffiths, R., White, N., \& Ghosh, P. 2001, ApJ, 559, L91

Ranalli, P., Comastri, A., \& Setti, G. 2003, A\&A, 399, 39

Reakes, M. 1980, MNRAS, 192, 297

Rephaeli, Y. \& Gruber, D. 2002, A\&A, 389, 752

Reynolds, A. P., Bell, S. A., \& Hilditch, R. W. 1992, MNRAS, 256, 631

Rice, W., Lonsdale, C. J., Soifer, B. T., et al. 1988, ApJS, 68, 91

Richards, E. A. 2000, ApJ, 533, 611

Richards, E. A., Kellermann, K. I., Fomalont, E. B., Windhorst, R. A., \& Partridge, R. B. 1998, AJ, 116, 1039

Ritter, H. \& Kolb, U. 1998, A\&AS, 129, 83

Roberts, T. P., Warwick, R. S., Ward, M. J., \& Murray, S. S. 2002, MNRAS, 337, 677

Rosa-González, D., Terlevich, E., \& Terlevich, R. 2002, MNRAS, 332, 283

Roussel, H., Sauvage, M., Vigroux, L., \& Bosma, A. 2001, A\&A, 372, 427

Rownd, B. K. \& Young, J. S. 1999, AJ, 118, 670

Sage, L. J. 1993, A\&A, 272, 123

Sandage, A. \& Tammann, G. A. 1980, A revised Shapley-Ames Catalog of bright galaxies (Washington: Carnegie Institution, 1980, Preliminary version)

Sazonov, S. Y., Sunyaev, R. A., \& Lund, N. 1997, Pis ma Astronomicheskii Zhurnal, 23, 326

Schreier, E., Giacconi, R., Gursky, H., Kellogg, E., \& Tananbaum, H. 1972, ApJ, 178, L71

Schulz, N., Canizares, C., Huenemoerder, D., et al. 2001, ApJ, 549, 441

Schurch, N. J., Roberts, T. P., \& Warwick, R. S. 2002, MNRAS, 335, 241

Shakura, N. \& Sunyaev, R. 1973, A\&A, 24, 337

Sharina, M. E., Karachentsev, I. D., \& Tikhonov, N. A. 1996, A\&AS, 119, 499

Shklovsky, I. S. 1967, ApJ, 148, L1+ 
Simonson, S. C. 1976, A\&A, 46, 261

Smale, A. P. 1998, ApJ, 498, L141

Smith, D. A., Morgan, E. H., \& Bradt, H. 1997, ApJ, 479, L137

Smith, D. S. \& Wilson, A. S. 2001, ApJ, 557, 180

Sobolev, V. 1949, Seria Matem. Nauk, 18, N1163

Sofue, Y., Reuter, H.-P., Krause, M., Wielebinski, R., \& Nakai, N. 1992, ApJ, 395, 126

Solomon, P., Sanders, D., \& Rivolo, A. 1985, ApJL, 292, 19

Soria, R. \& Kong, A. K. H. 2002, ApJ, 572, L33

Soria, R. \& Wu, K. 2002, A\&A, 384, 99

Spinoglio, L., Malkan, M. A., Rush, B., Carrasco, L., \& Recillas-Cruz, E. 1995, ApJ, 453, 616

Stergioulas, N. 1998, Living Rev. Relativity 1, 8, cited on 25.02.2003

Stern, D., Tozzi, P., Stanford, S. A., et al. 2002, AJ, 123, 2223

Strickland, D. K., Colbert, E. J. M., Heckman, T. M., et al. 2001, ApJ, 560, 707

Sugizaki, M., Mitsude, K., Kaneda, H., et al. 2001, ApJS, 134, 77

Sunyaev, R. 1990, IAUC, 5104, 1

Sunyaev, R., Gilfanov, M., Churazov, E., et al. 1990, Soviet Astronomy Letters, 16, 55+

Sunyaev, R. \& Revnivtsev, M. 2000, A\&A, 358, 617

Sunyaev, R. A., Tinsley, B. M., \& Meier, D. L. 1978, Comments on Astrophysics, 7, 183

Sunyaev, R. A. \& Titarchuk, L. G. 1985, A\&A, 143, 374

Tanaka, Y. 1992, in X-ray binaries and recycled pulsars, ed. E. van den Heuvel \& S. Rappaport, Vol. C 377 (Kluwer), 37

Tananbaum, H., Gursky, H., Kellogg, E., Giacconi, R., \& Jones, C. 1972, ApJ, 177, L5

Taylor, J. \& Cordes, J. 1993, ApJ, 411, 674

Terashima, Y. \& Wilson, A. 2002, astro-ph/0204321

Thronson, H. A., Hunter, D. A., Casey, S., Harper, D. A., \& Latter, W. B. 1989, ApJ, 339,803 
Toomre, A. \& Toomre, J. 1972, ApJ, 178, 623

Truemper, J., Pietsch, W., Reppin, C., et al. 1978, ApJ, 219, L105

Tsunemi, H., Kitamoto, S., Okamura, S., \& Roussel-Dupre, D. 1989, ApJ, 337, L81

Tutukov, A. V., Fedorova, A. V., Ergma, E. V., \& Yungelson, L. R. 1987, Soviet Astronomy Letters, 13, 328

Ueda, Y., Ishisaki, Y., Takahashi, T., Makishima, K., \& Ohashi, T. 2001, ApJS, 133, 1

Vallée, J. 1995, ApJ, 454, 119

van Paradijs, J. 1994, Cambridge Astrophysics Series, Vol. 26, X-ray binaries (Cambridge University Press), 536

van Paradijs, J. \& White, N. 1995, ApJ, 447, L33

Verbunt, F. 1996, in NATO ASIC Proc. 477: Evolutionary Processes in Binary Stars, 201

Verbunt, F. \& van den Heuvel, E. 1994, Cambridge Astrophysics Series, Vol. 26, X-ray binaries (Cambridge University Press), 457

Viallefond, F., Allen, R. J., \& de Boer, J. A. 1980, A\&A, 82, 207

Wachter, S. \& Margon, B. 1996, AJ, 112, 2684

Warwick, R., Marshall, N., Fraser, G., et al. 1981, MNRAS, 197, 865

Watson, M. G., Ricketts, M. J., \& Griffiths, R. E. 1978, ApJ, 221, L69

Webbink, R. F. 1985, in IAU Symp. 113: Dynamics of Star Clusters, Vol. 113, 541

Weisskopf, M. C., Tananbaum, H. D., Van Speybroeck, L. P., \& O’Dell, S. L. 2000, in Proc. SPIE Vol. 4012, p. 2-16, X-Ray Optics, Instruments, and Missions III, Joachim E. Truemper; Bernd Aschenbach; Eds., 2-16

Wen, L., Remillard, R. A., \& Bradt, H. V. 2000, ApJ, 532, 1119

White, N., Kaluzienski, J., \& Swank, J. 1984, in High Energy Transients in Astrophysics, ed. S. Woosley, AIP Conference Proceedings No. 115 (New York: American Institute of Physics), 31-48

White, N. E., Becker, R. H., Pravdo, S. H., et al. 1980, ApJ, 239, 655

White, N. E. \& van Paradijs, J. 1996, ApJ, 473, L25

Wilkinson, M. I. \& Evans, N. W. 1999, MNRAS, 310, 645

Williams, J. P. \& McKee, C. F. 1997, ApJ, 476, 166 
Yokogawa, J., Kensuke Imanishi, M. T., Nishiuchi, M., \& Koyama, K. 2000, ApJS, 128, 491

Young, J. S., Allen, L., Kenney, J. D. P., Lesser, A., \& Rownd, B. 1996, AJ, 112, 1903

Zezas, A., Fabbiano, G., Rots, A. H., \& Murray, S. S. 2002, ApJS, 142, 239

Zombeck, M. V. 1990, Handbook of Space Astronomy and Astrophysics, 2nd edn. (Cambridge University Press), 82 


\section{Acknowledgment}

I want to thank all people who made this thesis possible.

First of all I want to thank Rashid Sunyaev for the possibility to do a thesis at MPA and his unshakable enthusiasm. for astrophysics in general. Great debt I owe to Marat Gilfanov whose patience and expertise in the intricacies of astrophysical analysis were of great help during my stay.

Moreover I want to thank the colleagues at MPA and neighbouring institutes for always be willing to discuss problems, provide help and in general made for a very pleasant atmosphere. In particular this goes for my office mates, Markus Brueggen, Fritz Vollmer, Florian Siebel and Anna Gallazzi.

I am grateful to Fritz Vollmer and Greg Rudnick for the proof-reading of the manuscript.

Thanks also go to Benedetta Ciardi for organizing welcome breaks from work routine and her Italian lessons. And to Brice Menard, Jon Braithwaite and Lidia Tasca for their rather unsuccessful attempts to make me a mountaineer.

Special thanks go to my family for providing, before and during my $\mathrm{PhD}$ time, all support needed and then some. 


\title{
Curriculum vitae
}

\author{
Personal Hans-Jakob Grimm \\ Landwehrstr. 12 \\ D-80336 München, Germany
}

\section{Date and place of birth}

08. 06. 1973 in Rostock, Germany

\section{Education}

11/1999-08/2003 PhD thesis at Max-Planck-Institut für Astrophysik

Title: X-ray binaries in the Milky Way and other galaxies

Supervisor: Prof. Rashid Sunyaev

03/1998-09/1999 Diploma thesis (Institut für experimentelle Kermphysik)

Title: Investigation of the MSSM parameter space for exclusion of Higgs boson masses (in German)

Supervisor: Prof. Wim de Boer

Member of DELPHI Collaboration at CERN

10/1993-09/1999 Study of physics at Technische Universität, Karlsruhe

10/1992-09/1993 Military service

08/1974-05/1982 Gymasium, Lahr

08/1979-07/1983 Elementary school, Mahlberg 
